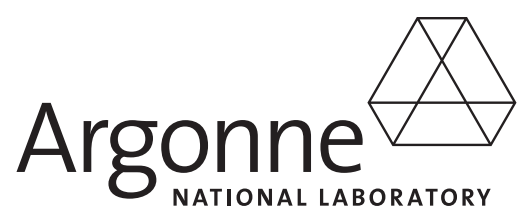

ANL/EVS/AGEM/TR-08-17

\title{
July 2008 Monitoring Results for Barnes, Kansas
}

\section{Environmental Science Division}


About Argonne National Laboratory

Argonne is a U.S. Department of Energy laboratory managed by UChicago Argonne, LLC under contract DE-AC02-06CH11357. The Laboratory's main facility is outside Chicago, at 9700 South Cass Avenue, Argonne, Illinois 60439. For information about Argonne, see www.anl.gov.

\section{Availability of This Report}

This report is available, at no cost, at http://www.osti.gov/bridge. It is also available on paper to the U.S. Department of Energy and its contractors, for a processing fee, from:

U.S. Department of Energy

Office of Scientific and Technical Information

P.O. Box 62

Oak Ridge, TN 37831-0062

phone (865) 576-8401

fax (865) 576-5728

reports@adonis.osti.gov

\section{Disclaimer}

This report was prepared as an account of work sponsored by an agency of the United States Government. Reference herein to any specific commercial product, process, or service by trade name, trademark, manufacturer, or otherwise, does not necessarily constitute or imply its endorsement, recommendation, or favoring by the United States Government or any agency thereof. The views and opinions of document authors expressed herein do not necessarily state or reflect those of the United States Government or any agency thereof, Argonne National Laboratory, or UChicago Argonne, LLC. 


\section{July 2008 Monitoring Results for Barnes, Kansas}

by

Applied Geosciences and Environmental Management Section

Environmental Science Division, Argonne National Laboratory

September 2008

USDA 


\section{Contents}

Notation

1 Introduction and Background.............................................................................. 1-1

2 Sampling and Analysis Activities ……………………............................................... 2-1

2.1 Measurement of Groundwater Levels ................................................................... 2-1

2.2 Monitoring Well Sampling and Analyses .............................................................. 2-2

2.3 Handling and Disposal of Investigation-Derived Waste......................................... 2-. 2-4

2.4 Quality Control for Sample Collection, Handling, and Analysis............................. 2-4

3 Results and Discussion.......................................................................................... 3-1

3.1 Groundwater Level Data ............................................................................... 3-1

3.2 Analytical Results for Volatile Organic Compounds in Groundwater Samples and Lateral Distribution of the Contaminants.....

3.3 Vertical Distribution of Carbon Tetrachloride and Evaluation of Data to Distinguish Aquifer Zones by Depth

4 Conclusions and Ongoing Tasks ............................................................................... 4- 4

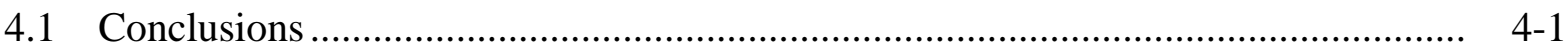

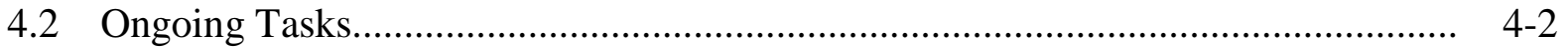

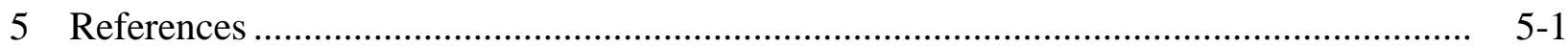

Appendix A： Sampling Activities and Field Measurements at Barnes in July 2008 ............. A-1

Appendix B: Results from the AGEM Laboratory for Dual Analyses of Samples Collected at Barnes in July 2008 and for Quality Control Samples................. B-1

Appendix C: Sample Documentation from TestAmerica Laboratories, Inc........................... C- C-1

\section{Figures}

1.1 Groundwater sampling locations at Barnes in July 2008

2.1 Wells at Barnes equipped with data loggers for water level monitoring, as of December 19, 2007, and May 9, 2008

3.1 Potentiometric surface maps depicting the groundwater flow direction at Barnes in December 2007 and May 2008, before pumping of the public water supply wells and during pumping

3.2 Hydrographs summarizing monthly results of long-term water level monitoring in wells at Barnes, January to July 2008. 


\section{Figures (Cont.)}

3.3 Analytical results for carbon tetrachloride in groundwater samples collected at Barnes in July-August 2006 and March-April 2007

3.4 Analytical results for carbon tetrachloride in groundwater samples collected at Barnes in November 2007 and March 2008.

3.5 Analytical results for carbon tetrachloride in groundwater samples collected at Barnes in March 2008 and July 2008

3.6 Analytical results for chloroform in groundwater samples collected at Barnes in November 2007 and March 2008.

3.7 Analytical results for chloroform in groundwater samples collected at Barnes in July 2008

3.8 Interpreted carbon tetrachloride plume in July 2008 in wells screened in the intermediate aquifer zone

3.9 Interpreted carbon tetrachloride plume in July 2008 in wells screened in the deep aquifer zone

\section{Tables}

3.1 Hand-measured water levels at Barnes, November 2007, December 2007, March 2008, and July 2008

3.2 Analytical results from the AGEM Laboratory for volatile organic compounds in groundwater samples collected at Barnes, July 2006 to July 2008

A.1 Sequence of sampling activities at Barnes in July 2008

A.2 Field measurements for groundwater samples collected at Barnes, July 2006 to July 2008

B.1 Analytical results for samples and replicates collected at Barnes in July 2008 and for quality control samples 


\section{Notation}

AGEM Applied Geosciences and Environmental Management

AMSL above mean sea level

BGL below ground level

${ }^{\circ} \mathrm{C} \quad$ degree(s) Celsius

CCC Commodity Credit Corporation

COC chain of custody

DO dissolved oxygen

EPA U.S. Environmental Protection Agency

$\mathrm{ft} \quad$ foot (feet)

gal gallon(s)

KDHE Kansas Department of Health and Environment

L liter(s)

$\mu \mathrm{g} / \mathrm{L} \quad \operatorname{microgram}(\mathrm{s})$ per liter

$\mu \mathrm{S} / \mathrm{cm} \quad$ microsiemen(s) per centimeter

$\mathrm{mg} / \mathrm{L} \quad$ milligram(s) per liter

min minute

$\mathrm{mV} \quad$ millivolt(s)

ORP oxidation-reduction potential

RBSL risk-based screening level

TOC top of casing

USDA U.S. Department of Agriculture

VOC volatile organic compound 


\section{July 2008 Monitoring Results for Barnes, Kansas}

\section{Introduction and Background}

The Commodity Credit Corporation of the U.S. Department of Agriculture (CCC/USDA) operated a grain storage facility at Barnes, Kansas, during most of the interval 1949-1974. Carbon tetrachloride contamination was initially detected in 1986 in the town's public water supply wells. In 2006-2007, the CCC/USDA conducted a comprehensive targeted investigation at and near its former property in Barnes to characterize this contamination. Those results were reported previously (Argonne 2008a).

In November 2007, the CCC/USDA began quarterly groundwater monitoring at Barnes. The monitoring is being conducted on behalf of the CCC/USDA by Argonne National Laboratory, in accord with the recommendations made in the report for the 2006-2007 targeted investigation (Argonne 2008a). The objective is to monitor the carbon tetrachloride contamination identified in the groundwater at Barnes. The sampling is presently conducted in a network of 28 individual monitoring wells (at 19 distinct locations), 2 public water supply wells, and 1 private well (Figure 1.1).

The results of the 2006-2007 targeted investigation and the subsequent monitoring events in November 2007 (Argonne 2008b) and March 2008 (Argonne 2008c) demonstrated the presence of carbon tetrachloride contamination in groundwater at levels slightly exceeding the Kansas Department of Health and Environment (KDHE) Tier 2 risk-based screening level (RBSL) of $5.0 \mu \mathrm{g} / \mathrm{L}$ for this compound. The contaminant plume appears to extend from the former CCC/USDA property northwestward, toward the Barnes public water supply wells. Information obtained during the 2006-2007 investigations indicates that at least one other potential source might have contributed to the groundwater contaminant plume (Argonne 2008a).

This current report presents the results of the third monitoring event, conducted in July 2008. During this third monitoring event, low-flow sampling methods were used to purge and sample all wells. This was the second event at Barnes during which low-flow sampling methods were used. 


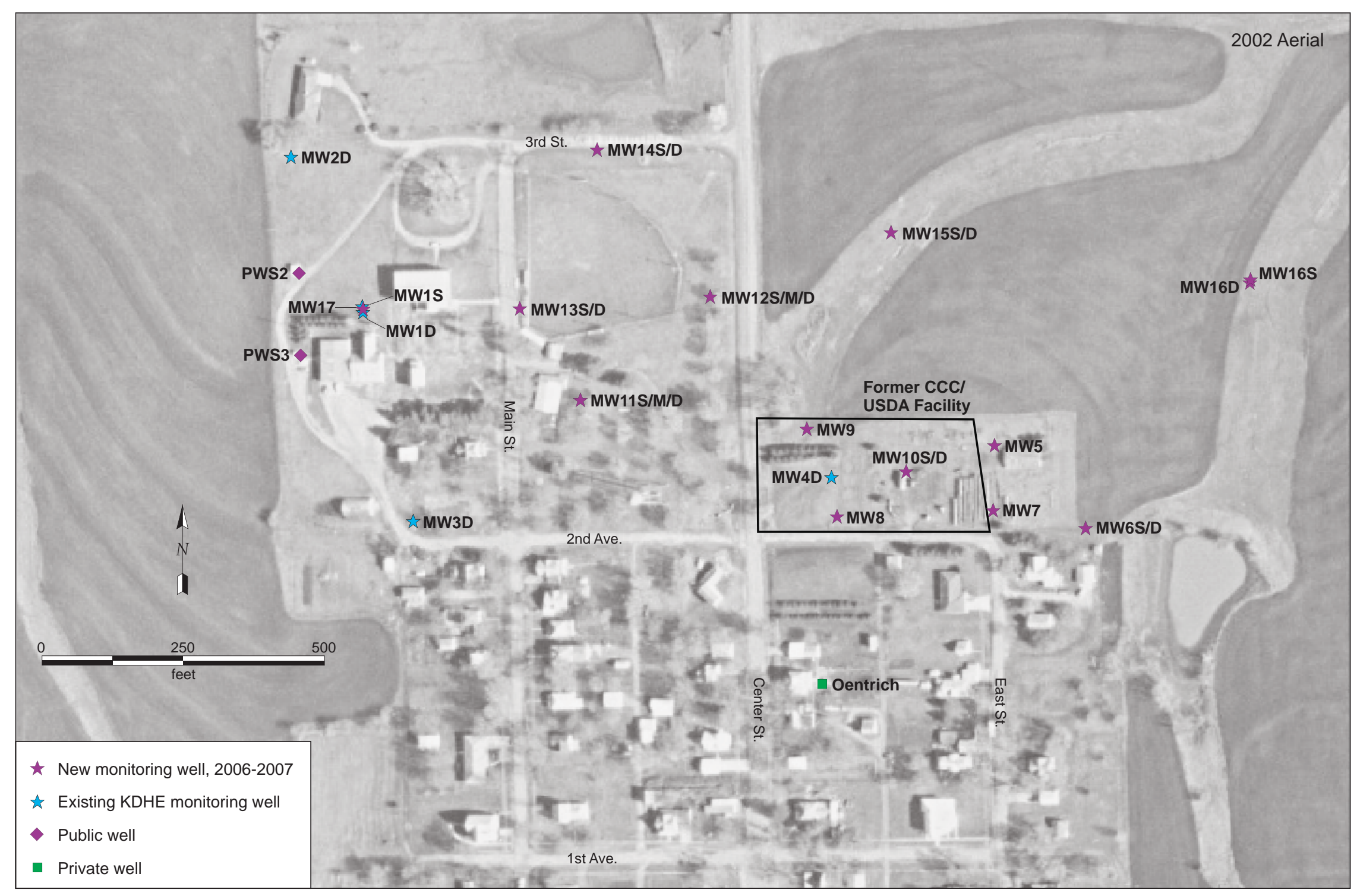

FIGURE 1.1 Groundwater sampling locations at Barnes in July 2008. Source of photograph: NAPP (2002). 


\section{Sampling and Analysis Activities}

\subsection{Measurement of Groundwater Levels}

The groundwater sampling event at Barnes on July 9-12, 2008, involved 28 monitoring wells (MW1S, MW1D, MW2D, MW3D, MW4D, MW5, MW6S, MW6D, MW7, MW8, MW9, MW10S, MW10D, MW11S, MW11M, MW11D, MW12S, MW12M, MW12D, MW13S, MW13D, MW14S, MW14D, MW15S, MW15D, MW16S, MW16D, MW17). All of the well locations are shown in Figure 1.1. A chronological summary of the field activities is in Appendix A, Table A.

Before implementation of the low-flow sampling described in Section 2.2, a hand-held water level indicator was used to measure the depth to groundwater and the total depth of each well, to within $0.01 \mathrm{ft}$, from the top of the well casing. Monitoring wells MW1S and MW12S were measured but were found to be dry and consequently could not be sampled. Two public water supply wells (PWS2 and PWS3) and one private well (Oentrich) were sampled but could not be measured because of well construction issues. The hand-measured water level data are presented in Section 3.1 and discussed in Section 3.3.

In addition to the manual water level measurements, data recorders have been gathering long-term data on the groundwater elevation and gradient at monitoring wells MW1D, MW2D, MW3D, MW4D, MW7, MW9, MW14D, MW15D, and MW16D and at the Oentrich private well (Figure 2.1). The data loggers record water levels continuously at 30-min intervals. During the current monitoring period, the data loggers were downloaded on July 9, 2008.

During the download on July 9, 2008, problems were discovered that had caused the following data recorders to stop working:

- The data recorder in well MW3D was found to be damaged, probably because of water infiltrating into the well vault. The recorder was subsequently removed and replaced.

- The battery for the data recorder in well MW7 had failed prematurely on June 25, 2008. The battery was replaced. 
- The data recorder at the Oentrich private well was found to have been removed on May 9, 2008. The recorder was removed when the property owner repaired the well pump and installed a new sanitary seal that the limits access to the well. The data recorder could not be replaced.

Currently, nine recorders are installed and working properly (all locations in Figure 2.1 except for the Oentrich well). The automatically recorded groundwater level data are presented and discussed in Section 3.1.

\subsection{Monitoring Well Sampling and Analyses}

After measurement of water levels, low-flow groundwater sampling techniques, according to U.S. Environmental Protection Agency (EPA) guidelines (Puls and Barcelona 1996), were used to purge and sample the monitoring wells. The Oentrich well and the public water supply wells were sampled at their respective faucets. The Oentrich well was pumped for 5 min before sampling; the public water supply wells were pumped for $0.5 \mathrm{~h}$ (Table A.1 in Appendix A).

The low-flow sampling of monitoring wells involved the use of a bladder pump and field measurement equipment designed to determine when representative formation water was entering the well casing. Stabilization of formation water in the screened area of the well was determined by measuring the static water levels and monitoring the levels of $\mathrm{pH}$, temperature, specific conductivity, oxidation-reduction potential (ORP), and dissolved oxygen (DO) during pumping.

The following procedure was followed for each well sampled:

1. A bladder pump was inserted into the well to a depth midway between the top and bottom of the screen. To minimize disturbance of the solids that are typically present at the bottom of a well, care was taken not to lower the equipment or pump to the bottom of the well screen. 
2. The pumping rate for the bladder pump was set to ensure that minimal drawdown occurred in each well during pumping. The rate was monitored by measuring the static water level continuously throughout pumping.

3. Poly tubing was used to connect the bladder pump and to an in-line flow cell. Formation parameters, including $\mathrm{pH}$, temperature, specific conductivity, ORP, and DO, were measured continuously in the in-line flow cell during pumping. Measurements were recorded every 4 min until 3 successive measurements for each parameter were within a range indicating that the formation water was stable. The range for formation stabilization varies for each parameter, as follows: $\mathrm{pH}$, within 0.1 ; temperature, within $3 \%$; specific conductivity, within $3 \%$; ORP, within $10 \mathrm{mV}$; and DO, within $10 \%$.

4. After formation stabilization occurred, the poly tubing was disconnected from the in-line flow cell, and a representative groundwater sample was transferred from the poly tubing into laboratory-approved containers.

5. Poly tubing for each well was kept and dedicated for reuse at that well. In addition, pumping rate data were recorded for each well as a reference for subsequent sampling events.

The sequence of activities during the July 2008 well sampling event is summarized in Appendix A, Table A.1. The field measurements are in Appendix A, Table A.2.

Groundwater samples designated for analyses for volatile organic compounds (VOCs) were collected in appropriate laboratory containers, labeled, packaged, and chilled to $4^{\circ} \mathrm{C}$ by placement in ice-filled coolers. The samples were shipped via an overnight delivery service to the Applied Geosciences and Environmental Management (AGEM) Laboratory at Argonne for VOCs analyses with EPA Method 524.2 (EPA 1995). Aliquots of selected samples (chosen in the field) were also shipped to TestAmerica Laboratories, Inc., South Burlington, Vermont, for verification VOCs analyses according to EPA Contract Laboratory Program protocols.

The analytical results are presented and discussed in Section 3.2. 


\subsection{Handling and Disposal of Investigation-Derived Waste}

Purge water generated as potentially contaminated investigation-derived waste was containerized on-site in 55-gal drums. The accumulated purge water was sampled and analyzed for VOCs (including ethylene dibromide) and nitrates. The analytical results indicated no detectable concentrations of carbon tetrachloride, chloroform, or ethylene dibromide. Nitrate was present at $3.7 \mathrm{mg} / \mathrm{L}$. With the approval of the KDHE (2008a), the water was discharged on-site on July 31, 2008.

\subsection{Quality Control for Sample Collection, Handling, and Analysis}

Quality assurance/quality control procedures followed during the July 2008 monitoring event are described in detail in the Master Work Plan (Argonne 2002). The results are summarized as follows:

- Sample collection and handling activities were monitored by the documentation of samples as they were collected and the use of chain-ofcustody forms and custody seals to ensure sample integrity during handling and shipment.

- Samples designated for VOCs analyses were received with custody seals intact and at the appropriate preservation temperature. All samples sent to the AGEM Laboratory were analyzed within the required holding times.

- Quality control samples collected to monitor sample-handling activities (trip blanks and equipment rinsates) and method blanks analyzed with the samples to monitor analytical methodologies were all free of carbon tetrachloride and chloroform contamination.

- Groundwater samples were analyzed for VOCs at the AGEM Laboratory by the purge-and-trap method and a gas chromatograph-mass spectrometer system. Calibration checks analyzed with each sample delivery group were required to be within $\pm 20 \%$ of the standard. Surrogate standard determinations 
performed on samples and blanks were within the specified range of $80-120 \%$ for all samples, in either the initial analysis or a successful reanalysis.

- Results from the AGEM Laboratory for dual analyses of the groundwater samples are in Appendix B, Table B.1. The results of the dual analyses compare well, indicating consistency in the sampling and analytical methodologies. Analytical results for quality control samples are also in Appendix B, Table B.1.

- In accordance with the procedures defined in the Master Work Plan (Argonne 2002), five groundwater samples were submitted to a second laboratory (TestAmerica) for verification analysis according to the protocols of the EPA's Contract Laboratory Program. Documentation is in Appendix C. The results from the two laboratories compare well over the range of contaminant concentrations detected, with average relative percent difference values of $10.7 \%$ for carbon tetrachloride and $12.0 \%$ for chloroform. Methylene chloride was not detected by either laboratory. The trace detection of carbon tetrachloride in the sample from public well PWS3 was confirmed in verification analysis. 


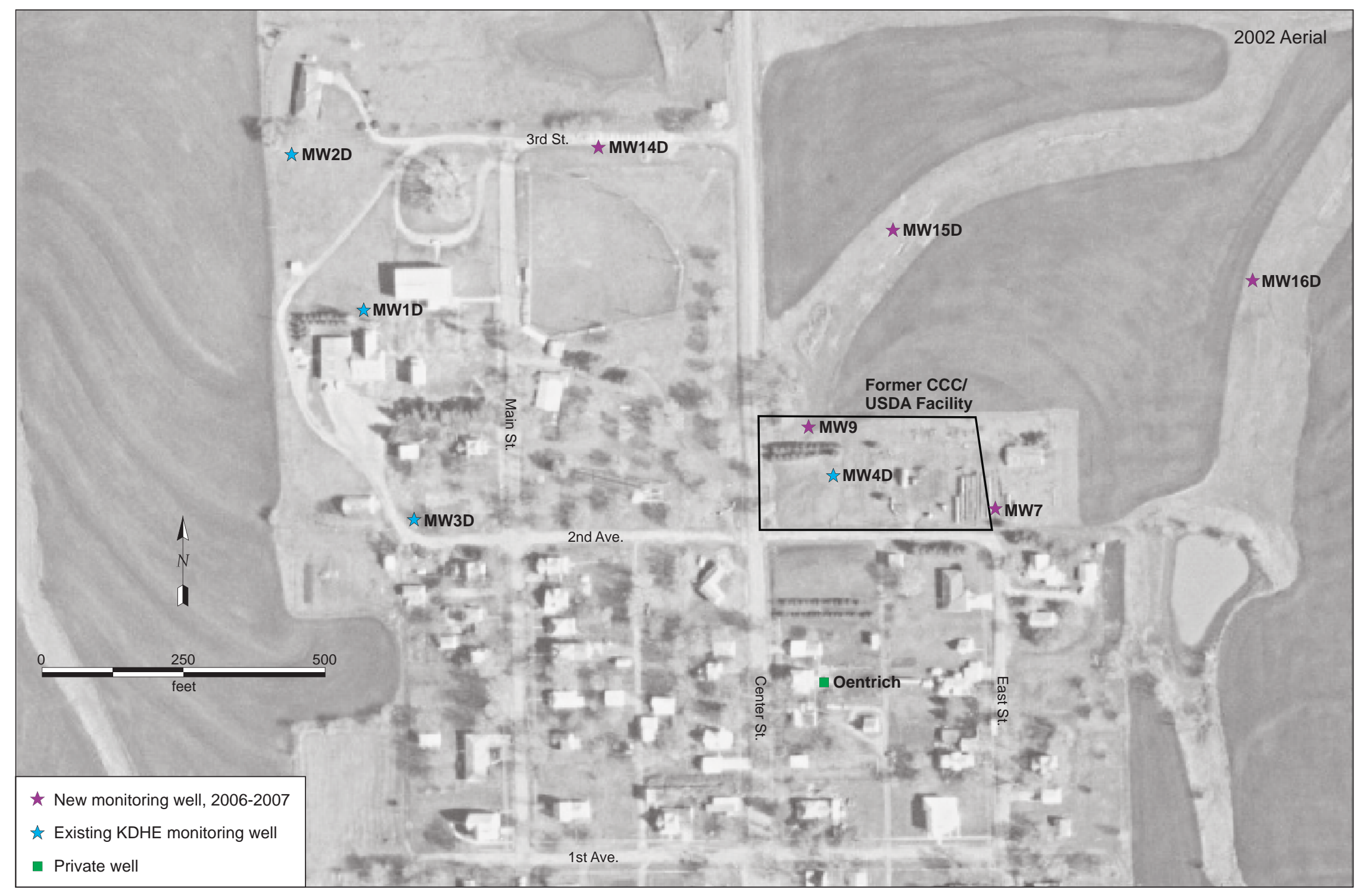

FIGURE 2.1 Wells at Barnes equipped with data loggers for water level monitoring, as of December 19, 2007, and May 9, 2008. Source of photograph: NAPP (2002). 


\section{Results and Discussion}

\subsection{Groundwater Level Data}

The manual water level measurements taken during sampling on July 9-12, 2008, are in Table 3.1. These measurements are discussed in Section 3.3. Table 3.1 also includes manual water level measurements made in May 2007-April 2008.

Figure 3.1 presents water level contours for December 19, 2007, and May 9, 2008, for the network of recording transducers installed for long-term water level monitoring at Barnes. The network on those dates included wells MW1D, MW2D, MW3D, MW4D, MW7, MW9, MW14D, MW15D, MW16D, and the Oentrich private well. The Oentrich well was removed from the network later on May 9, 2008, when the property owner replaced the pump and installed a new sanitary seal that limits access to the well (see Section 2.1).

Extensive documentation of the potentiometric surface at Barnes during the targeted investigation (Argonne 2008a) and subsequent monitoring events indicated that operation of the public water supply wells strongly influences the groundwater flow direction. The more recent contours for the network, for May 9, 2008 (Figure 3.1), continue to show that flow directions are significantly different before pumping begins (top) versus during pumping (bottom).

The flow directions shown for May 9, 2008 (Figure 3.1), are easterly prior to pumping of the public wells and northwesterly during periods of pumping. Previous data (for example, the contours for December 19, 2007, also in Figure 3.1) have indicated a northeasterly direction prior to pumping and a northwesterly direction during pumping. The apparent change during non-pumping hours in the present monitoring period might have resulted from the absence of data from the southern control point at MW3D. During the data download on July 9, 2008, the data logger in MW3D was found to be damaged and not working. The data could not be recovered. The recorder has now been replaced (Section 2.1). With control reestablished at MW3D, subsequent sampling events should either confirm the apparent change in flow direction or demonstrate that the absence of this important data collection point affected the interpretation of groundwater flow direction.

The hydrographs in Figure 3.2 summarize data for the period January 1, 2008, to July 9, 2008, for the recording transducers. As indicated in Section 2.1, the recorder in well MW3D 
malfunctioned, and thus no trace for this well appears in Figure 3.2. In addition, the battery in the well MW7 recorder failed on June 25, 2008, and the recorder for the Oentrich well was removed on May 9, 2008. The traces for these two wells end on these respective dates.

The water level data for well MW15D indicate a persistent pattern of higher groundwater levels. This pattern of higher water levels in a deeper well might indicate that vertical hydraulic gradients are present.

The patterns in Figure 3.2 are similar to patterns recorded in 2007 during the targeted investigation and in subsequent monitoring events (Argonne 2008a,b,c). The hydrographs show responses to pumping of the public water supply wells that are characterized by drawdowns of as much as $2.25 \mathrm{ft}$ during pumping, with almost immediate rebound when pumping stops. The most prominent drawdowns are consistently observed in wells MW1D and MW2D, which are the closest monitoring points to the public wells.

In 2007, water levels at Barnes rose by approximately 7-8 ft (Argonne 2008a,b). During January 2008, the levels remained relatively stable. Subsequently, the levels increased through July 9, 2008, showing an overall increase of 6.5-11 ft since January 2008 (Figure 3.2).

\subsection{Analytical Results for Volatile Organic Compounds in Groundwater Samples and Lateral Distribution of the Contaminants}

The analytical data for VOCs in the groundwater samples collected in July 2008 are in Table 3.2, together with data for the previous sampling events at Barnes. The lateral distribution of carbon tetrachloride in groundwater in the July-August 2006 and March-April 2007 sampling events is illustrated in Figure 3.3, while the November 2007 and March 2008 results are illustrated in Figure 3.4. The July 2008 distribution is illustrated in Figure 3.5, with the March 2008 data for comparison. The overall carbon tetrachloride distribution patterns are similar for the five events.

Overall, carbon tetrachloride was detected in July 2008 in 21 of the 29 groundwater samples collected (at 14 of the 20 distinct monitoring locations; Figure 3.5 and Table 3.2). Carbon tetrachloride concentrations in the 29 samples were as follows: 
- Not detected at an instrument detection limit of $0.1 \mu \mathrm{g} / \mathrm{L}$ in 8 samples (MW2D, MW3D, MW5, MW6S, MW11S, MW15D, MW16S, and MW16D).

- Trace levels below the method quantitation limit of $1.0 \mu \mathrm{g} / \mathrm{L}$ in 9 samples (MW1D, MW6D, MW11D, MW12D, MW14D, MW17, PWS2, PWS3, and the Oentrich private well).

- Concentrations of $1.0-4.9 \mu \mathrm{g} / \mathrm{L}$ in 5 samples (MW7, MW9, MW10D, MW11M, and MW15S).

- Concentrations at or above the RBSL value of $5.0 \mu \mathrm{g} / \mathrm{L}$ in 7 samples (MW4D, MW8, MW10S, MW12M, MW13S, MW13D, and MW14S).

The maximum carbon tetrachloride concentration detected was $49 \mu \mathrm{g} / \mathrm{L}$ (at MW10S, on the former CCC/USDA property).

In November 2007 and March 2008 (Figure 3.6), trace to low concentrations of chloroform were found in association with the more elevated carbon tetrachloride concentrations. In July 2008, chloroform concentrations ranging from trace levels to $4.2 \mu \mathrm{g} / \mathrm{L}$ (at MW12M) were detected in 12 of the 29 groundwater samples (at 9 of the 20 distinct sampling locations; Table 3.2 and Figure 3.7). As in the November 2007 and March 2008 sampling events, in July 2008 chloroform occurred in association with the more elevated carbon tetrachloride levels identified (at MW4D, MW8, MW10S, MW12M, MW13S, MW13D, and MW14S in July 2008).

The lateral distribution of carbon tetrachloride in groundwater in July 2008 was similar to the distribution observed during previous sampling events in 2007 and 2008, with a slight increase in contaminant migration near the public water supply wells. These wells showed trace concentrations of carbon tetrachloride, below the method quantitation limit (estimated at $0.8 \mu \mathrm{g} / \mathrm{L}$ in PWS2 and $0.2 \mu \mathrm{g} / \mathrm{L}$ in PWS3), during the July 2008 sampling event. This marks the first observation since July 2000 (PWS2) or March 2007 (PWS3) of detectable concentrations of carbon tetrachloride in these wells.

The most significant changes noted in carbon tetrachloride distribution since the March 2008 sampling were observed in wells MW4D and MW13D. The carbon tetrachloride concentrations decreased from March 2008 to July 2008 in wells MW4D (on the former 
CCC/USDA property; from $18 \mu \mathrm{g} / \mathrm{L}$ to $9.4 \mu \mathrm{g} / \mathrm{L}$ ) and MW13D (northwest of the former CCC/USDA property and adjacent to the high school; from $11 \mu \mathrm{g} / \mathrm{L}$ to $5.9 \mu \mathrm{g} / \mathrm{L}$ ).

In the sentinel wells, as defined previously (Section 5.1 of Argonne 2008a), carbon tetrachloride was detected at trace concentrations below the method quantitation limit of $1.0 \mu \mathrm{g} / \mathrm{L}$ in wells MW1D, MW6D, MW14D, and MW17. No carbon tetrachloride was detected (at an instrument detection limit of $0.1 \mu \mathrm{g} / \mathrm{L}$ ) in sentinel wells MW2D, MW3D, MW6S, MW15D, MW16S, and MW16D. Sentinel wells that showed slight increases in concentrations of carbon tetrachloride include MW14S and MW15S.

\subsection{Vertical Distribution of Carbon Tetrachloride and Evaluation of Data to Distinguish Aquifer Zones by Depth}

The designations "S," "M," and "D" in monitoring well names were assigned at the time of well installation. They indicate shallow, medium, and deep screens (relative depths) in an individual well, rather than the aquifer zone screened. After the March 2008 monitoring, a detailed evaluation of the hand-measured water levels (Table 3.1) and VOCs data (Table 3.2) was conducted in an attempt to differentiate aquifer zones by depth. The data collected in July 2008 were evaluated similarly. The data from both events suggest at least three vertically distinct zones within the aquifer. The aquifer zones are as follows:

- Shallow Aquifer Zone. The only monitoring wells believed to be completed in the shallow zone are MW1S, MW11S, and MW12S. Wells MW1S and MW12S were dry during the March and July 2008 sampling events; well MW11S showed a water level elevation of approximately 1,312 ft AMSL in the July event.

- Intermediate Aquifer Zone. The intermediate zone is believed to be represented by monitoring wells MW10S, MW11M, MW12M, MW13S, and MW17. Water level elevations measured manually for these wells in July 2008 were approximately 1,255-1,258 ft AMSL.

- Deep Aquifer Zone. Monitoring wells believed to represent the deep zone include MW1D, MW2D, MW3D, MW4D, MW5, MW6S, MW6D, MW7, MW8, MW9, MW10D, MW11D, MW12D, MW13D, MW14S, MW14D, 
MW15S, MW15D, MW16S, and MW16D. The water level elevations measured manually in these monitoring wells in July 2008 were approximately 1,229-1,239 ft AMSL. On the basis of its hand-measured water level in November 2007 (approximately 1,220 ft AMSL [Table 3.2] and the automatic measurements [Figure 3.2]), the Oentrich well is also considered to be screened in the deep aquifer zone. All of the wells equipped with data loggers for water level monitoring are screened in the deep zone.

The vertical distribution of the carbon tetrachloride in groundwater indicates that the highest concentrations were detected in the intermediate zone, at wells MW10S (49 $\mu \mathrm{g} / \mathrm{L})$, MW12M $(27 \mu \mathrm{g} / \mathrm{L})$, and MW13S $(17 \mu \mathrm{g} / \mathrm{L})$. The deep-zone wells at these locations showed lower concentrations $(3.9 \mu / \mathrm{L}$ at MW10D, a trace [estimated at $0.7 \mu \mathrm{g} / \mathrm{L}$ ] at MW12D, and $5.9 \mu \mathrm{g} / \mathrm{L}$ at MW13D). Figures 3.8 and 3.9 illustrated the interpreted contaminant distributions in the intermediate and deep zones, respectively. The shallow-zone well (MW11S) continued to show no detectable concentrations of carbon tetrachloride (Table 3.2 and Figure 3.5). 
TABLE 3.1 Hand-measured water levels at Barnes, November 2007, December 2007, March 2008, and July 2008.

\begin{tabular}{|c|c|c|c|c|c|c|c|c|c|}
\hline \multirow[b]{3}{*}{ Well } & \multirow{3}{*}{$\begin{array}{l}\text { Reference } \\
\text { Elevation } \\
\text { (ft AMSL) }\end{array}$} & \multicolumn{8}{|c|}{ Water Level on Date Indicated } \\
\hline & & \multicolumn{2}{|c|}{$11 / 18-19 / 07$} & \multicolumn{2}{|c|}{$12 / 19 / 07$} & \multicolumn{2}{|c|}{ 3/4-10/08 } & \multicolumn{2}{|c|}{ 7/9-12/08 } \\
\hline & & $\mathrm{ft} \mathrm{TOC}^{\mathrm{a}}$ & ft AMSL & $\mathrm{ft} \mathrm{TOC}^{\mathrm{a}}$ & ft AMSL & ft $\mathrm{TOC}^{\mathrm{a}}$ & ft AMSL & ft TOC $^{\mathrm{a}}$ & ft AMSL \\
\hline \multicolumn{10}{|c|}{ Shallow aquifer zone } \\
\hline $\begin{array}{l}\text { MW1S } \\
\text { MW11S } \\
\text { MW12S }\end{array}$ & $\begin{array}{l}1351.58 \\
1336.58 \\
1327.46\end{array}$ & $\begin{array}{l}\text { Dry } \\
29.20 \\
\text { Dry }\end{array}$ & $\begin{array}{c}- \\
1307.38 \\
-\end{array}$ & Dry & - & $\begin{array}{c}\text { Dry } \\
27.70 \\
\text { Dry }\end{array}$ & $\begin{array}{c}- \\
1308.88 \\
-\end{array}$ & $\begin{array}{l}\text { Dry } \\
24.80 \\
\text { Dry }\end{array}$ & $\begin{array}{c}- \\
1311.78 \\
-\end{array}$ \\
\hline \multicolumn{10}{|c|}{ Intermediate aquifer zone } \\
\hline $\begin{array}{l}\text { MW10S } \\
\text { MW11M } \\
\text { MW12M } \\
\text { MW13S } \\
\text { MW17 }\end{array}$ & $\begin{array}{l}1331.33 \\
1336.51 \\
1327.46 \\
1342.36 \\
1351.77\end{array}$ & $\begin{array}{r}77.81 \\
82.33 \\
74.50 \\
92.23 \\
102.68\end{array}$ & $\begin{array}{l}1253.52 \\
1254.18 \\
1252.96 \\
1250.13 \\
1249.09\end{array}$ & & & $\begin{array}{r}77.47 \\
82.65 \\
74.77 \\
92.10 \\
101.75\end{array}$ & $\begin{array}{l}1253.86 \\
1253.86 \\
1252.69 \\
1250.26 \\
1250.02\end{array}$ & $\begin{array}{l}73.40 \\
78.85 \\
70.10 \\
87.00 \\
96.60\end{array}$ & $\begin{array}{l}1257.93 \\
1257.66 \\
1257.36 \\
1255.36 \\
1255.17\end{array}$ \\
\hline \multicolumn{10}{|c|}{ Deep aquifer zone } \\
\hline MW1D & 1351.33 & 124.89 & 1226.44 & 125.54 & 1225.79 & 127.66 & 1223.67 & 119.40 & 1231.93 \\
\hline MW2D & 1348.85 & 122.56 & 1226.29 & 123.20 & 1225.65 & 125.55 & 1223.30 & 117.15 & 1231.70 \\
\hline MW3D & 1345.99 & 126.25 & 1219.74 & 120.09 & 1225.90 & 121.90 & 1224.09 & 113.30 & 1232.69 \\
\hline MW4D & 1326.32 & 101.39 & 1224.93 & 100.54 & 1225.78 & 101.74 & 1224.58 & 93.60 & 1232.72 \\
\hline MW5 & 1327.20 & 102.78 & 1224.42 & & & 102.00 & 1225.20 & 93.80 & 1233.40 \\
\hline MW6S & 1323.13 & 96.10 & 1227.03 & & & 94.50 & 1228.63 & 88.10 & 1235.03 \\
\hline MW6D & 1323.15 & 98.50 & 1224.65 & & & 98.50 & 1224.65 & 89.50 & 1233.65 \\
\hline MW7 & 1329.91 & 105.50 & 1224.41 & 104.48 & 1225.43 & 105.62 & 1224.29 & 97.50 & 1232.41 \\
\hline MW8 & 1330.06 & 105.17 & 1224.89 & & & 104.38 & 1225.68 & 95.75 & 1234.31 \\
\hline MW9 & 1321.86 & 97.20 & 1224.66 & 96.25 & 1225.61 & 96.40 & 1225.46 & 87.65 & 1234.21 \\
\hline MW10D & 1331.33 & 106.22 & 1225.11 & & & 106.36 & 1224.97 & 97.30 & 1234.03 \\
\hline MW11D & 1336.53 & 112.46 & 1224.07 & & & 110.50 & 1226.03 & 102.10 & 1234.43 \\
\hline MW12D & 1327.52 & 102.00 & 1225.52 & & & 103.30 & 1224.22 & 93.70 & 1233.82 \\
\hline MW13D & 1342.37 & 117.83 & 1224.54 & & & 118.19 & 1224.18 & 107.90 & 1234.47 \\
\hline MW14S & 1332.69 & 106.75 & 1225.94 & & & 106.95 & 1225.74 & 99.40 & 1233.29 \\
\hline MW14D & 1332.74 & 107.10 & 1225.64 & 107.23 & 1225.51 & 106.95 & 1225.79 & 101.00 & 1231.74 \\
\hline MW15S & 1309.34 & 84.33 & 1225.01 & & & 84.66 & 1224.68 & 80.30 & 1229.04 \\
\hline MW15D & 1309.29 & 70.20 & 1239.09 & 80.84 & 1228.45 & 80.80 & 1228.49 & 70.30 & 1238.99 \\
\hline MW16S & 1299.47 & 75.30 & 1224.17 & & & 75.50 & 1223.97 & 67.35 & 1232.12 \\
\hline MW16D & 1299.52 & 74.50 & 1225.02 & 74.62 & 1224.90 & 75.00 & 1224.52 & 66.30 & 1233.22 \\
\hline Oentrich $^{b}$ & 1336.93 & $\mathrm{NM}^{\mathrm{C}}$ & & 116.55 & 1220.38 & NM & NM & NM & NM \\
\hline
\end{tabular}

a TOC, top of casing.

b The Oentrich well water level was measured from the concrete at the top of the well vault. The value shown was corrected by $5.5 \mathrm{ft}$ to give a measured depth from the top of the casing.

c NM, not measured (sampled from faucet). 
TABLE 3.2 Analytical results from the AGEM Laboratory for volatile organic compounds in groundwater samples collected at Barnes, July 2006 to July 2008.

\begin{tabular}{|c|c|c|c|c|c|c|}
\hline \multirow[b]{2}{*}{ Location } & \multirow[b]{2}{*}{$\begin{array}{l}\text { Screen } \\
\text { Interval } \\
\text { (ft BGL) }\end{array}$} & \multirow[b]{2}{*}{ Sample } & \multirow[b]{2}{*}{$\begin{array}{l}\text { Sample } \\
\text { Date }\end{array}$} & \multicolumn{3}{|c|}{ Concentration $(\mu \mathrm{g} / \mathrm{L})$} \\
\hline & & & & $\begin{array}{c}\text { Carbon } \\
\text { Tetrachloride }\end{array}$ & Chloroform & $\begin{array}{c}\text { Methylene } \\
\text { Chloride }\end{array}$ \\
\hline \multicolumn{7}{|c|}{ Previously existing KDHE monitoring wells } \\
\hline MW1S & 13.3-23.3 & $\begin{array}{l}\text { Not sampled (well dry) } \\
\text { Not sampled (well dry) } \\
\text { Not sampled (well dry) } \\
\text { Not sampled (well dry) } \\
\text { Not sampled (well dry) }\end{array}$ & $\begin{array}{r}7 / 19 / 06 \\
4 / 4 / 07 \\
11 / 18 / 07 \\
3 / 4 / 08 \\
7 / 9 / 08\end{array}$ & $\begin{array}{l}- \\
- \\
- \\
- \\
-\end{array}$ & $\begin{array}{l}- \\
- \\
- \\
- \\
-\end{array}$ & $\begin{array}{l}- \\
- \\
- \\
- \\
-\end{array}$ \\
\hline MW1D & $139.85-159.4$ & $\begin{array}{l}\text { BAMW1D-W-21688 } \\
\text { BAMW1D-W-22565 } \\
\text { BAMW1D-W-22593 } \\
\text { BAMW1D-W-22627 } \\
\text { BAMW1D-W-22668 }\end{array}$ & $\begin{array}{r}7 / 19 / 06 \\
4 / 4 / 07 \\
11 / 18 / 07 \\
3 / 4 / 08 \\
7 / 9 / 08\end{array}$ & $\begin{array}{l}1.0 \\
1.2 \\
N D \\
0.2 \mathrm{Jb} \\
0.2 \mathrm{~J}\end{array}$ & $\begin{array}{l}N D^{a} \\
N D \\
N D \\
N D \\
N D\end{array}$ & $\begin{array}{l}\text { ND } \\
\text { ND } \\
\text { ND } \\
\text { ND } \\
\text { ND }\end{array}$ \\
\hline MW2D & $133.26-152.93$ & $\begin{array}{l}\text { BAMW2D-W-21687 } \\
\text { BAMW2D-W-22564 } \\
\text { BAMW2D-W-22594 } \\
\text { BAMW2D-W-22628 } \\
\text { BAMW2D-W-22669 }\end{array}$ & $\begin{array}{r}7 / 19 / 06 \\
4 / 4 / 07 \\
11 / 18 / 07 \\
3 / 7 / 08 \\
7 / 10 / 08\end{array}$ & $\begin{array}{l}\text { ND } \\
\text { ND } \\
\text { ND } \\
\text { ND } \\
\text { ND }\end{array}$ & $\begin{array}{l}\text { ND } \\
\text { ND } \\
\text { ND } \\
\text { ND } \\
\text { ND }\end{array}$ & $\begin{array}{l}\text { ND } \\
\text { ND } \\
\text { ND } \\
\text { ND } \\
\text { ND }\end{array}$ \\
\hline MW3D & $133.02-152.73$ & $\begin{array}{l}\text { BAMW3D-W-21686 } \\
\text { BAMW3D-W-22567 } \\
\text { BAMW3D-W-22595 } \\
\text { BAMW3D-W-22629 } \\
\text { BAMW3D-W-22670 }\end{array}$ & $\begin{array}{r}7 / 19 / 06 \\
4 / 4 / 07 \\
11 / 19 / 07 \\
3 / 7 / 08 \\
7 / 10 / 08\end{array}$ & $\begin{array}{l}\text { ND } \\
\text { ND } \\
\text { ND } \\
\text { ND } \\
\text { ND }\end{array}$ & $\begin{array}{l}\text { ND } \\
\text { ND } \\
\text { ND } \\
\text { ND } \\
\text { ND }\end{array}$ & $\begin{array}{l}\text { ND } \\
\text { ND } \\
\text { ND } \\
\text { ND } \\
\text { ND }\end{array}$ \\
\hline MW4D & $98.38-118.22$ & $\begin{array}{l}\text { BAMW4D-W-21690 } \\
\text { BAMW4D-W-22583 } \\
\text { BAMW4D-W-22596 } \\
\text { BAMW4D-W-22642 } \\
\text { BAMW4D-W-22671 }\end{array}$ & $\begin{array}{r}7 / 20 / 06 \\
4 / 6 / 07 \\
11 / 19 / 07 \\
3 / 9 / 08 \\
7 / 12 / 08\end{array}$ & $\begin{array}{c}2.1 \\
3.5 \\
1.7 \\
18 \\
9.4\end{array}$ & $\begin{array}{l}\text { ND } \\
0.1 \mathrm{~J} \\
0.4 \mathrm{~J} \\
0.4 \mathrm{~J} \\
0.5 \mathrm{~J}\end{array}$ & $\begin{array}{l}\text { ND } \\
\text { ND } \\
\text { ND } \\
\text { ND } \\
\text { ND }\end{array}$ \\
\hline CCC/USL & ells installed dur & ng the 2006-2007 investi & & & & \\
\hline MW5 & $110-120$ & $\begin{array}{l}\text { BAMW5-W-22589 } \\
\text { BAMW5-W-22597 } \\
\text { BAMW5-W-22637 } \\
\text { BAMW5-W-22672 }\end{array}$ & $\begin{array}{r}4 / 6 / 07 \\
11 / 19 / 07 \\
3 / 8 / 08 \\
7 / 11 / 08\end{array}$ & $\begin{array}{l}0.6 \mathrm{~J} \\
0.6 \mathrm{~J} \\
0.7 \mathrm{~J} \\
N D\end{array}$ & $\begin{array}{l}\text { ND } \\
\text { ND } \\
\text { ND } \\
\text { ND }\end{array}$ & $\begin{array}{l}\text { ND } \\
\text { ND } \\
\text { ND } \\
\text { ND }\end{array}$ \\
\hline MW6S & $90.5-100.5$ & $\begin{array}{l}\text { Not sampled (well dry) } \\
\text { BAMW6S-W-22598 } \\
\text { BAMW6S-W-22635 } \\
\text { BAMW6S-W-22673 }\end{array}$ & $\begin{array}{r}4 / 4 / 07 \\
11 / 19 / 07 \\
3 / 8 / 08 \\
7 / 11 / 08\end{array}$ & $\begin{array}{l}0.3 \mathrm{~J} \\
0.4 \mathrm{~J} \\
\mathrm{ND}\end{array}$ & $\begin{array}{l}\text { ND } \\
\text { ND } \\
\text { ND }\end{array}$ & $\begin{array}{l}\text { ND } \\
\text { ND } \\
\text { ND }\end{array}$ \\
\hline MW6D & $105-115$ & $\begin{array}{l}\text { BAMW6D-W-22573 } \\
\text { BAMW6D-W-22599 } \\
\text { BAMW6D-W-22636 } \\
\text { BAMW6D-W-22674 }\end{array}$ & $\begin{array}{r}4 / 5 / 07 \\
11 / 19 / 07 \\
3 / 8 / 08 \\
7 / 11 / 08\end{array}$ & $\begin{array}{l}\text { ND } \\
0.5 \mathrm{~J} \\
0.8 \mathrm{~J} \\
0.9 \mathrm{~J}\end{array}$ & $\begin{array}{l}\text { ND } \\
\text { ND } \\
\text { ND } \\
\text { ND }\end{array}$ & $\begin{array}{l}\text { ND } \\
\text { ND } \\
\text { ND } \\
\text { ND }\end{array}$ \\
\hline
\end{tabular}


TABLE 3.2 (Cont.)

\begin{tabular}{|c|c|c|c|c|c|c|}
\hline \multirow[b]{2}{*}{ Location } & \multirow{2}{*}{$\begin{array}{l}\text { Screen } \\
\text { Interval } \\
\text { (ft BGL) }\end{array}$} & \multirow[b]{2}{*}{ Sample } & \multirow[b]{2}{*}{$\begin{array}{c}\text { Sample } \\
\text { Date }\end{array}$} & \multicolumn{3}{|c|}{ Concentration $(\mu / L)$} \\
\hline & & & & $\begin{array}{c}\text { Carbon } \\
\text { Tetrachloride }\end{array}$ & Chloroform & $\begin{array}{c}\text { Methylene } \\
\text { Chloride }\end{array}$ \\
\hline
\end{tabular}

CCC/USDA wells installed during the 2006-2007 investigation (cont.)

\begin{tabular}{|c|c|c|c|c|c|}
\hline MW7 & $116-126$ & $\begin{array}{l}\text { BAMW7-W-22588 } \\
\text { BAMW7-W-22600 } \\
\text { BAMW7-W-22643 } \\
\text { BAMW7-W-22675 }\end{array}$ & $\begin{array}{r}4 / 6 / 07 \\
11 / 19 / 07 \\
3 / 9 / 08 \\
7 / 12 / 08\end{array}$ & $\begin{array}{l}1.0 \\
2.6 \\
2.8 \\
1.7\end{array}$ & $\begin{array}{l}\text { ND } \\
\text { ND } \\
\text { ND } \\
\text { ND }\end{array}$ \\
\hline MW8 & $110-120$ & $\begin{array}{l}\text { BAMW8-W-22584 } \\
\text { BAMW8-W-22601 } \\
\text { BAMW8-W-22652 } \\
\text { BAMW8-W-22676 }\end{array}$ & $\begin{array}{r}4 / 6 / 07 \\
11 / 19 / 07 \\
3 / 10 / 08 \\
7 / 11 / 08\end{array}$ & $\begin{array}{l}14 \\
23 \\
19 \\
21\end{array}$ & $\begin{array}{l}0.7 \mathrm{~J} \\
0.6 \mathrm{~J} \\
0.6 \mathrm{~J} \\
0.6 \mathrm{~J}\end{array}$ \\
\hline MW9 & $100-110$ & $\begin{array}{l}\text { BAMW9-W-22582 } \\
\text { BAMW9-W-22602 } \\
\text { BAMW9-W-22647 } \\
\text { BAMW9-W-22678 }\end{array}$ & $\begin{array}{r}4 / 5 / 07 \\
11 / 19 / 07 \\
3 / 9 / 08 \\
7 / 11 / 08\end{array}$ & $\begin{array}{l}1.0 \\
7.7 \\
3.0 \\
1.3\end{array}$ & $\begin{array}{l}\text { ND } \\
0.6 \mathrm{~J} \\
0.3 \mathrm{~J} \\
0.3 \mathrm{~J}\end{array}$ \\
\hline MW10S & $93-103$ & $\begin{array}{l}\text { BAMW10S-W-22586 } \\
\text { BAMW10S-W-22603 } \\
\text { BAMW10S-W-22649 } \\
\text { BAMW10S-W-22679 }\end{array}$ & $\begin{array}{r}4 / 6 / 07 \\
11 / 19 / 07 \\
3 / 10 / 08 \\
7 / 11 / 08\end{array}$ & $\begin{array}{l}20 \\
11 \\
56 \\
49\end{array}$ & $\begin{array}{l}1.4 \\
0.7 \mathrm{~J} \\
2 \\
1.8\end{array}$ \\
\hline MW10D & $115-125$ & $\begin{array}{l}\text { BAMW10D-W-22585 } \\
\text { BAMW10D-W-22604 } \\
\text { BAMW10D-W-22646 } \\
\text { BAMW10D-W-22680 }\end{array}$ & $\begin{array}{r}4 / 6 / 07 \\
11 / 19 / 07 \\
3 / 9 / 08 \\
7 / 11 / 08\end{array}$ & $\begin{array}{l}2.4 \\
6.3 \\
5.7 \\
3.9\end{array}$ & $\begin{array}{l}0.2 \mathrm{~J} \\
0.5 \mathrm{~J} \\
0.5 \mathrm{~J} \\
0.7 \mathrm{~J}\end{array}$ \\
\hline MW11S & $40-50$ & $\begin{array}{l}\text { BAMW11S-W-22570 } \\
\text { BAMW11S-W-22605 } \\
\text { BAMW11S-W-22630 } \\
\text { BAMW11S-W-22681 }\end{array}$ & $\begin{array}{r}4 / 4 / 07 \\
11 / 19 / 07 \\
3 / 5 / 08 \\
7 / 10 / 08\end{array}$ & $\begin{array}{l}\text { ND } \\
\text { ND } \\
\text { ND } \\
\text { ND }\end{array}$ & $\begin{array}{l}1.1 \\
0.6 \mathrm{~J} \\
0.6 \mathrm{~J} \\
0.4 \mathrm{~J}\end{array}$ \\
\hline MW11M & $90-100$ & $\begin{array}{l}\text { BAMW11M-W-22572 } \\
\text { BAMW11M-W-22606 } \\
\text { BAMW11M-W-22644 } \\
\text { BAMW11M-W-22682 }\end{array}$ & $\begin{array}{r}4 / 5 / 07 \\
11 / 19 / 07 \\
3 / 6 / 08 \\
7 / 10 / 08\end{array}$ & $\begin{array}{l}\text { ND } \\
3.7 \\
2.4 \\
2.4\end{array}$ & $\begin{array}{l}\text { ND } \\
\text { ND } \\
0.5 \mathrm{~J} \\
0.7 \mathrm{~J}\end{array}$ \\
\hline MW11D & $125-135$ & $\begin{array}{l}\text { BAMW11D-W-22571 } \\
\text { BAMW11D-W-22607 } \\
\text { BAMW11D-W-22639 } \\
\text { BAMW11D-W-22683 }\end{array}$ & $\begin{array}{r}4 / 4 / 07 \\
11 / 19 / 07 \\
3 / 5 / 08 \\
7 / 10 / 08\end{array}$ & $\begin{array}{l}1.1 \\
0.8 \mathrm{~J} \\
0.4 \mathrm{~J} \\
0.9 \mathrm{~J}\end{array}$ & $\begin{array}{l}\text { ND } \\
\text { ND } \\
\text { ND } \\
\text { ND }\end{array}$ \\
\hline MW12S & $43-50$ & $\begin{array}{l}\text { Not sampled (well dry) } \\
\text { Not sampled (well dry) } \\
\text { Not sampled (well dry) } \\
\text { Not sampled (well dry) }\end{array}$ & $\begin{array}{r}4 / 5 / 07 \\
11 / 19 / 07 \\
3 / 10 / 08 \\
7 / 10 / 08\end{array}$ & & \\
\hline MW12M & $90-100$ & $\begin{array}{l}\text { BAMW12M-W-22580 } \\
\text { BAMW12M-W-22609 } \\
\text { BAMW12M-W-22651 } \\
\text { BAMW12M-W-22685 }\end{array}$ & $\begin{array}{r}4 / 5 / 07 \\
11 / 19 / 07 \\
3 / 10 / 08 \\
7 / 10 / 08\end{array}$ & $\begin{array}{l}20 \\
18 \\
18 \\
27\end{array}$ & $\begin{array}{l}4.2 \\
5.1 \\
2.6 \\
4.2\end{array}$ \\
\hline
\end{tabular}


TABLE 3.2 (Cont.)

\begin{tabular}{|c|c|c|c|c|c|c|}
\hline \multirow[b]{2}{*}{ Location } & \multirow[b]{2}{*}{$\begin{array}{l}\text { Screen } \\
\text { Interval } \\
\text { (ft BGL) }\end{array}$} & \multirow[b]{2}{*}{ Sample } & \multirow[b]{2}{*}{$\begin{array}{c}\text { Sample } \\
\text { Date }\end{array}$} & \multicolumn{3}{|c|}{ Concentration $(\mu / L)$} \\
\hline & & & & $\begin{array}{c}\text { Carbon } \\
\text { Tetrachloride }\end{array}$ & Chloroform & $\begin{array}{c}\text { Methylene } \\
\text { Chloride }\end{array}$ \\
\hline
\end{tabular}

CCC/USDA wells installed during the 2006-2007 investigation (cont.)

\begin{tabular}{|c|c|c|c|c|c|}
\hline MW12D & $115-125$ & $\begin{array}{l}\text { BAMW12D-W-22576 } \\
\text { BAMW12D-W-22610 } \\
\text { BAMW12D-W-22641 } \\
\text { BAMW12D-W-22686 }\end{array}$ & $\begin{array}{r}4 / 5 / 07 \\
11 / 18 / 07 \\
3 / 9 / 08 \\
7 / 11 / 08\end{array}$ & $\begin{array}{l}0.6 \mathrm{~J} \\
1.6 \\
1.0 \\
0.7 \mathrm{~J}\end{array}$ & $\begin{array}{l}\text { ND } \\
\text { ND } \\
\text { ND } \\
\text { ND }\end{array}$ \\
\hline MW13S & $112-122$ & $\begin{array}{l}\text { BAMW13S-W-22575 } \\
\text { BAMW13S-W-22611 } \\
\text { BAMW13S-W-22650 } \\
\text { BAMW13S-W-22687 }\end{array}$ & $\begin{array}{r}4 / 5 / 07 \\
11 / 19 / 07 \\
3 / 10 / 08 \\
7 / 9 / 08\end{array}$ & $\begin{array}{l}21 \\
17 \\
17 \\
17\end{array}$ & $\begin{array}{l}1.6 \\
1.8 \\
1.5 \\
1.9\end{array}$ \\
\hline MW13D & $127-137$ & $\begin{array}{l}\text { BAMW13D-W-22574 } \\
\text { BAMW13D-W-22612 } \\
\text { BAMW13D-W-22645 } \\
\text { BAMW13D-W-22688 }\end{array}$ & $\begin{array}{r}4 / 5 / 07 \\
11 / 19 / 07 \\
3 / 9 / 08 \\
7 / 9 / 08\end{array}$ & $\begin{array}{c}3.5 \\
5.9 \\
11 \\
5.9\end{array}$ & $\begin{array}{l}0.4 \mathrm{~J} \\
0.2 \mathrm{~J} \\
1.1 \\
0.9 \mathrm{~J}\end{array}$ \\
\hline MW14S & $108-118$ & $\begin{array}{l}\text { BAMW14S-W-22569 } \\
\text { BAMW14S-W-22613 } \\
\text { BAMW14S-W-22640 } \\
\text { BAMW14S-W-22689 }\end{array}$ & $\begin{array}{r}4 / 4 / 07 \\
11 / 18 / 07 \\
3 / 8 / 08 \\
7 / 10 / 08\end{array}$ & $\begin{array}{l}0.9 \mathrm{~J} \\
1.2 \\
4.3 \\
5.6\end{array}$ & $\begin{array}{l}\text { ND } \\
\text { ND } \\
0.3 \mathrm{~J} \\
0.3 \mathrm{~J}\end{array}$ \\
\hline MW14D & $123-133$ & $\begin{array}{l}\text { BAMW14D-W-22568 } \\
\text { BAMW14D-W-22614 } \\
\text { BAMW14D-W-22638 } \\
\text { BAMW14D-W-22690 }\end{array}$ & $\begin{array}{r}4 / 4 / 07 \\
11 / 18 / 07 \\
3 / 8 / 08 \\
7 / 10 / 08\end{array}$ & $\begin{array}{l}1.2 \\
0.6 \mathrm{~J} \\
0.7 \mathrm{~J} \\
0.5 \mathrm{~J}\end{array}$ & $\begin{array}{l}\text { ND } \\
\text { ND } \\
\text { ND } \\
\text { ND }\end{array}$ \\
\hline MW15S & $88-98$ & $\begin{array}{l}\text { BAMW15S-W-22560 } \\
\text { BAMW15S-W-22615 } \\
\text { BAMW15S-W-22648 } \\
\text { BAMW15S-W-22691 }\end{array}$ & $\begin{array}{r}4 / 4 / 07 \\
11 / 18 / 07 \\
3 / 10 / 08 \\
7 / 12 / 08\end{array}$ & $\begin{array}{l}1.5 \\
8.7 \\
1.8 \\
2.2\end{array}$ & $\begin{array}{l}\text { ND } \\
0.4 \mathrm{~J} \\
0.2 \mathrm{~J} \\
0.3 \mathrm{~J}\end{array}$ \\
\hline MW15D & $105-115$ & $\begin{array}{l}\text { BAMW15D-W-22561 } \\
\text { BAMW15D-W-22616 } \\
\text { BAMW15D-W-22631 } \\
\text { BAMW15D-W-22692 }\end{array}$ & $\begin{array}{r}4 / 4 / 07 \\
11 / 18 / 07 \\
3 / 8 / 08 \\
7 / 12 / 08\end{array}$ & $\begin{array}{l}\text { ND } \\
\text { ND } \\
0.2 \mathrm{~J} \\
\text { ND }\end{array}$ & $\begin{array}{l}\text { ND } \\
\text { ND } \\
\text { ND } \\
\text { ND }\end{array}$ \\
\hline MW16S & $76-86$ & $\begin{array}{l}\text { BAMW16S-W-22563 } \\
\text { BAMW16S-W-22617 } \\
\text { BAMW16S-W-22632 } \\
\text { BAMW16S-W-22693 }\end{array}$ & $\begin{array}{r}4 / 4 / 07 \\
11 / 19 / 07 \\
3 / 7 / 08 \\
7 / 11 / 08\end{array}$ & $\begin{array}{l}\text { ND } \\
\text { ND } \\
0.4 \mathrm{~J} \\
\text { ND }\end{array}$ & $\begin{array}{l}\text { ND } \\
\text { ND } \\
\text { ND } \\
\text { ND }\end{array}$ \\
\hline MW16D & $90-100$ & $\begin{array}{l}\text { BAMW16D-W-22562 } \\
\text { BAMW16D-W-22618 } \\
\text { BAMW16D-W-22633 } \\
\text { BAMW16D-W-22694 }\end{array}$ & $\begin{array}{r}4 / 4 / 07 \\
11 / 19 / 07 \\
3 / 7 / 08 \\
7 / 11 / 08\end{array}$ & $\begin{array}{l}\text { ND } \\
\text { ND } \\
\text { ND } \\
\text { ND }\end{array}$ & $\begin{array}{l}\text { ND } \\
\text { ND } \\
\text { ND } \\
\text { ND }\end{array}$ \\
\hline MW17 & $120-130$ & $\begin{array}{l}\text { BAMW17D-W-22566 } \\
\text { BAMW17D-W-22619 } \\
\text { BAMW17-W-22634 } \\
\text { BAMW17-W-22695 }\end{array}$ & $\begin{array}{r}4 / 4 / 07 \\
11 / 19 / 07 \\
3 / 5 / 08 \\
7 / 9 / 08\end{array}$ & $\begin{array}{l}\text { ND } \\
\text { ND } \\
0.3 \mathrm{~J} \\
0.4 \mathrm{~J}\end{array}$ & $\begin{array}{l}\text { ND } \\
\text { ND } \\
\text { ND } \\
\text { ND }\end{array}$ \\
\hline
\end{tabular}


TABLE 3.2 (Cont.)

\begin{tabular}{|c|c|c|c|c|c|c|}
\hline \multirow[b]{2}{*}{ Location } & \multirow[b]{2}{*}{$\begin{array}{l}\text { Screen } \\
\text { Interval } \\
\text { (ft BGL) }\end{array}$} & \multirow[b]{2}{*}{ Sample } & \multirow[b]{2}{*}{$\begin{array}{l}\text { Sample } \\
\text { Date }\end{array}$} & \multicolumn{3}{|c|}{ Concentration $(\mu / L)$} \\
\hline & & & & $\begin{array}{c}\text { Carbon } \\
\text { Tetrachloride }\end{array}$ & Chloroform & $\begin{array}{l}\text { Methylene } \\
\text { Chloride }\end{array}$ \\
\hline \multicolumn{7}{|c|}{ Private wells } \\
\hline Oentrich & 150 & $\begin{array}{l}\text { BAOENT-W-21693 } \\
\text { BAOENT-W-21713 } \\
\text { BAOENTRICH-W-22579 } \\
\text { BAOENTRICH-W-22622 } \\
\text { BAOENTRICH-W-22654 } \\
\text { BAOENTRICH-W-22695 }\end{array}$ & $\begin{array}{r}7 / 20 / 06 \\
8 / 2 / 06 \\
4 / 5 / 07 \\
11 / 19 / 07 \\
3 / 6 / 08 \\
7 / 11 / 08\end{array}$ & $\begin{array}{l}0.3 \mathrm{~J} \\
0.6 \mathrm{~J} \\
0.6 \mathrm{~J} \\
0.8 \mathrm{~J} \\
1.3 \\
0.3 \mathrm{~J}\end{array}$ & $\begin{array}{l}\text { ND } \\
\text { ND } \\
\text { ND } \\
\text { ND } \\
\text { ND } \\
\text { ND }\end{array}$ & $\begin{array}{l}\text { ND } \\
\text { ND } \\
\text { ND } \\
\text { ND } \\
\text { ND } \\
\text { ND }\end{array}$ \\
\hline Sedivy & 138 & $\begin{array}{l}\text { BACW-W-21849 } \\
\text { BASED2-W-21913 }\end{array}$ & $\begin{array}{l}8 / 22 / 06 \\
9 / 13 / 06\end{array}$ & $\begin{array}{l}\text { ND } \\
\text { ND }\end{array}$ & $\begin{array}{l}\text { ND } \\
\text { ND }\end{array}$ & $\begin{array}{l}\text { ND } \\
\text { ND }\end{array}$ \\
\hline Sedivy1 & 90 & Not sampled (well dry) & $9 / 13 / 06$ & - & - & - \\
\hline \multicolumn{7}{|c|}{ Public water supply wells } \\
\hline PWS2 & 155 & $\begin{array}{l}\text { BAPWS2-W-22510 } \\
\text { BAPW2-W-22578 } \\
\text { BAPW2-W-22620 } \\
\text { BAPWS2-W-22655 } \\
\text { BAPWS2-W-22696 }\end{array}$ & $\begin{array}{r}3 / 9 / 07 \\
4 / 5 / 07 \\
11 / 20 / 07 \\
3 / 6 / 08 \\
7 / 11 / 08\end{array}$ & $\begin{array}{l}\text { ND } \\
\text { ND } \\
\text { ND } \\
\text { ND } \\
0.8 \mathrm{~J}\end{array}$ & $\begin{array}{l}\text { ND } \\
\text { ND } \\
\text { ND } \\
\text { ND } \\
\text { ND }\end{array}$ & $\begin{array}{l}\text { ND } \\
\text { ND } \\
\text { ND } \\
\text { ND } \\
\text { ND }\end{array}$ \\
\hline PWS3 & 160 & $\begin{array}{l}\text { BAPWS3-W-22511 } \\
\text { BAPW3-W-22577 } \\
\text { BAPW3-W-22621 } \\
\text { BAPWS3-W-22656 } \\
\text { BAPWS3-W-22697 }\end{array}$ & $\begin{array}{r}3 / 9 / 07 \\
4 / 5 / 07 \\
11 / 20 / 07 \\
3 / 6 / 08 \\
7 / 11 / 08\end{array}$ & $\begin{array}{l}0.2 \mathrm{~J} \\
\mathrm{ND} \\
\mathrm{ND} \\
\mathrm{ND} \\
0.2 \mathrm{~J}\end{array}$ & $\begin{array}{l}\text { ND } \\
\text { ND } \\
\text { ND } \\
\text { ND } \\
\text { ND }\end{array}$ & $\begin{array}{l}\text { ND } \\
\text { ND } \\
\text { ND } \\
\text { ND } \\
\text { ND }\end{array}$ \\
\hline
\end{tabular}

a ND, contaminant not detected at an instrument detection limit of $0.1 \mu \mathrm{g} / \mathrm{L}$.

b Qualifier $\mathrm{J}$ indicates an estimated concentration below the purge-and-trap method quantitation limit of $1.0 \mu \mathrm{g} / \mathrm{L}$. 

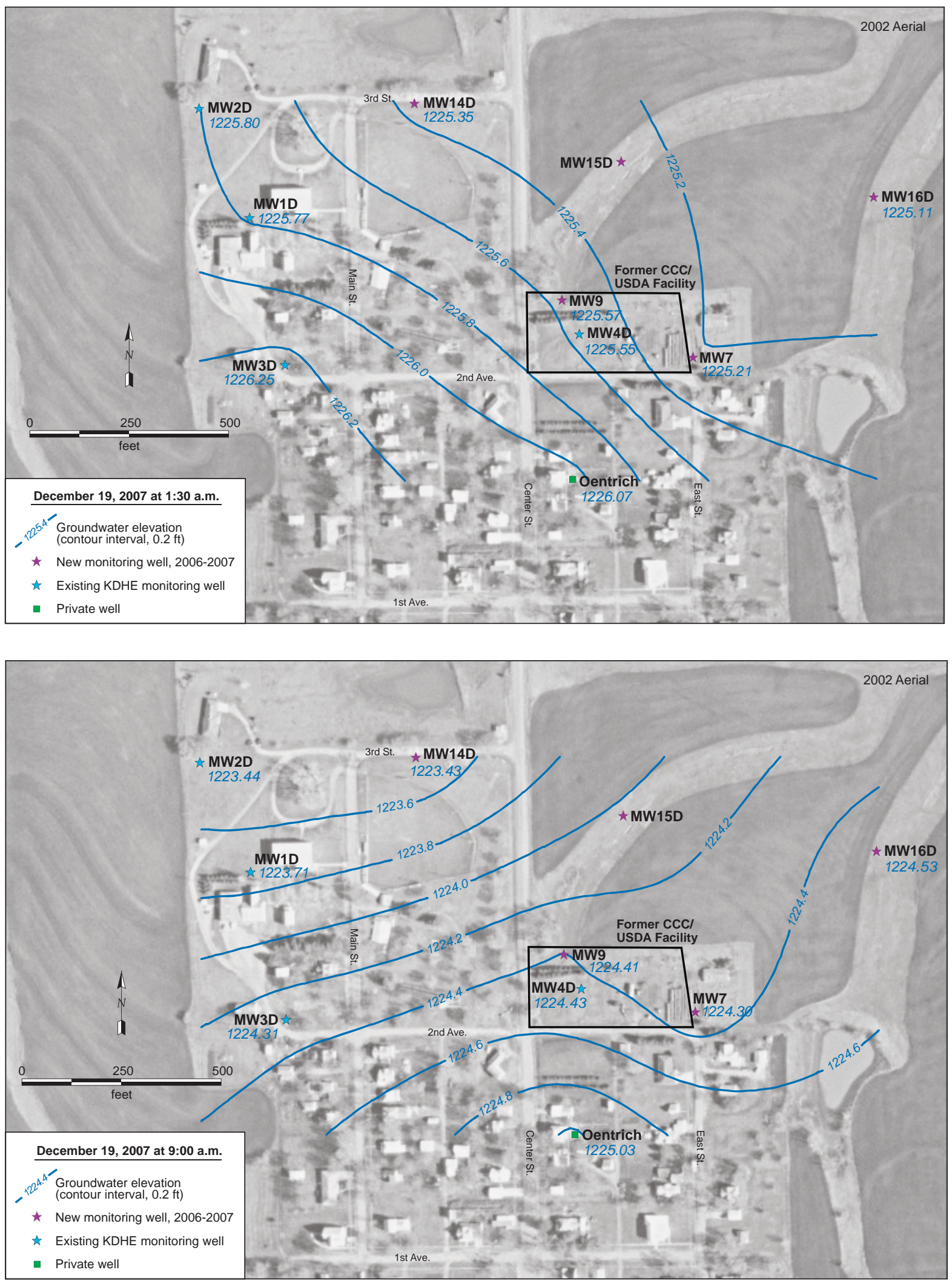

FIGURE 3.1 Potentiometric surface maps depicting the groundwater flow direction at Barnes in December 2007 and May 2008, before pumping of the public water supply wells (top panels) and during pumping (bottom panels). Source of photograph: NAPP (2002). 

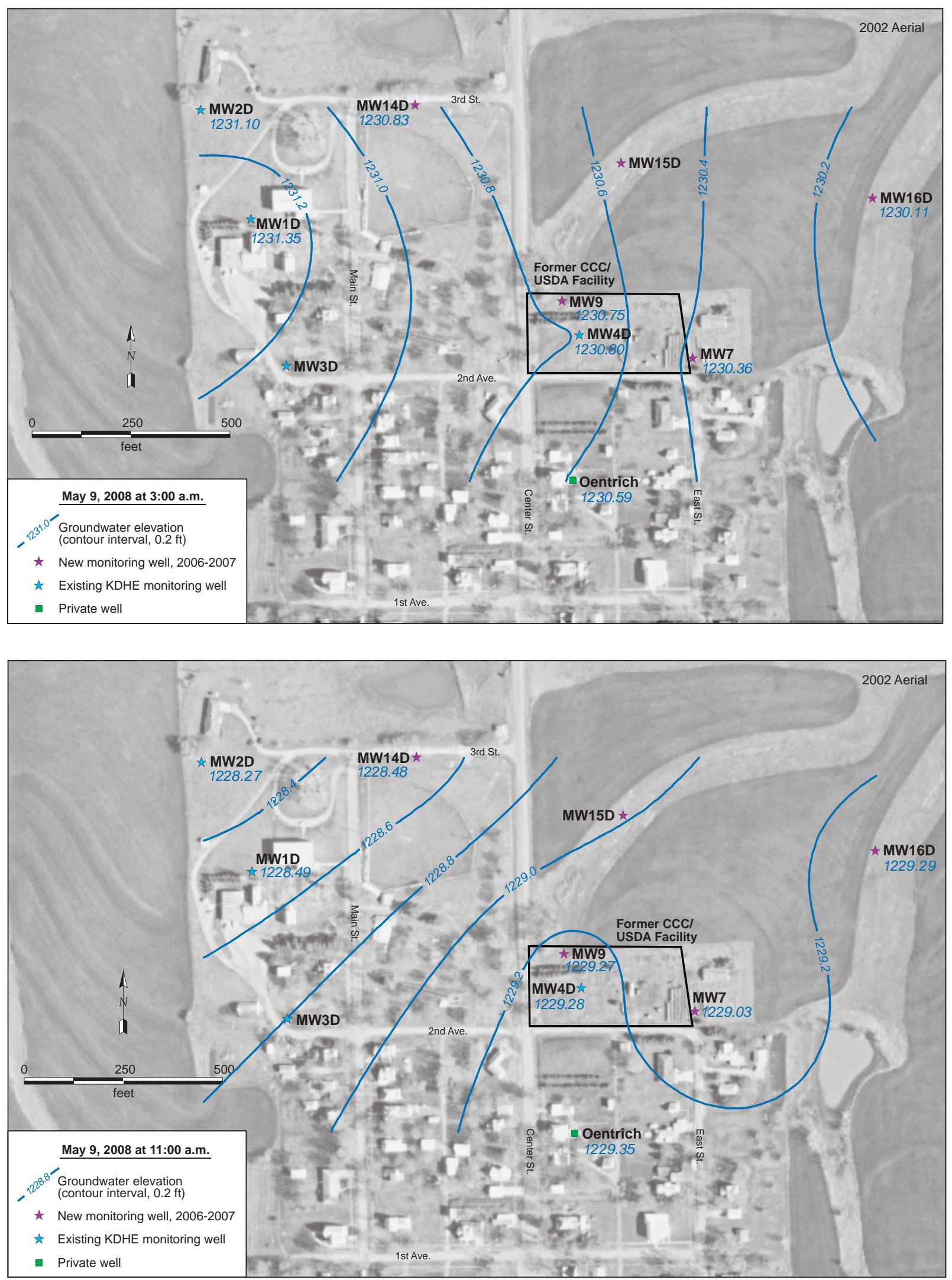

FIGURE 3.1 (Cont.) 

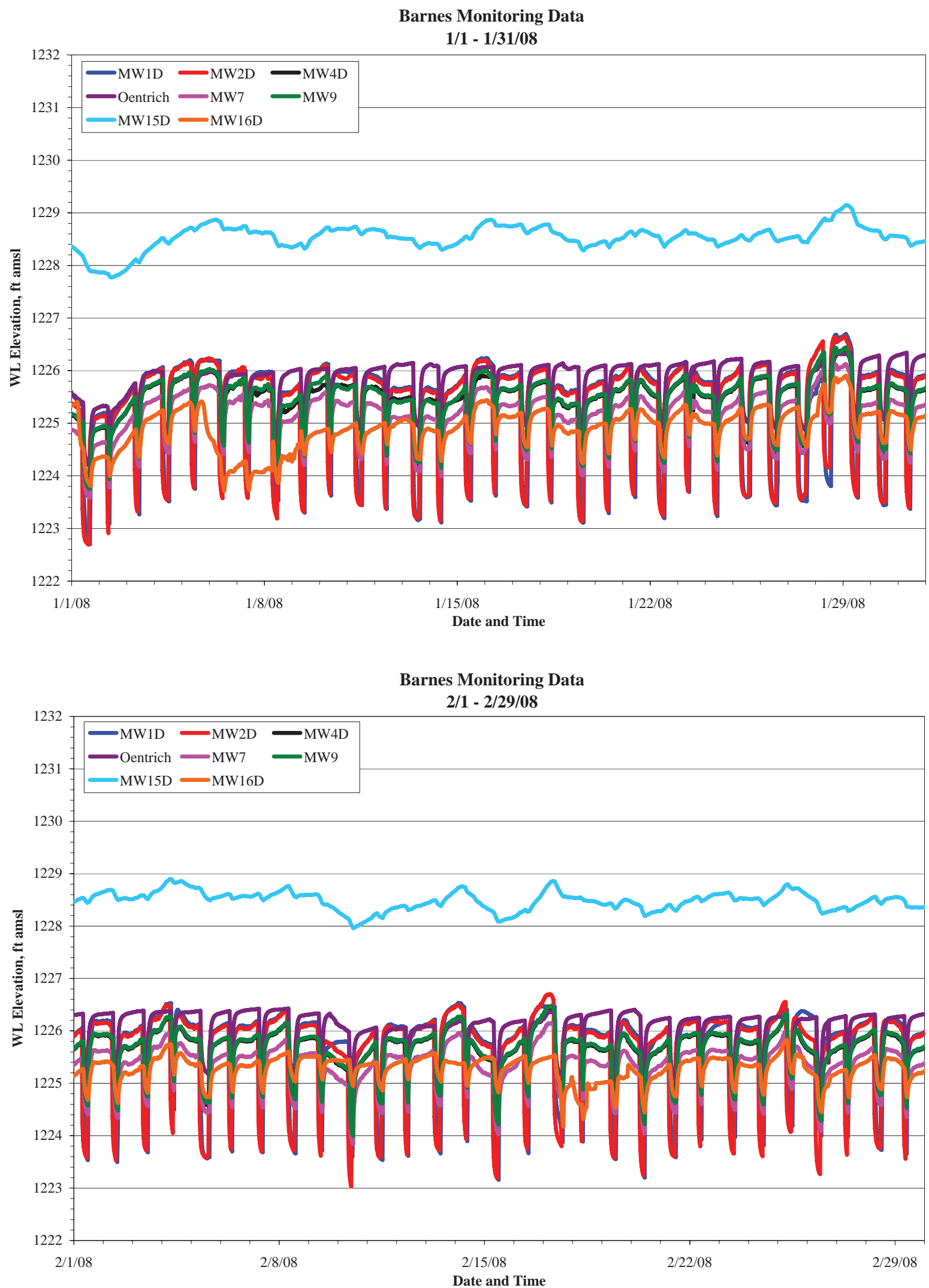

FIGURE 3.2 Hydrographs summarizing monthly results of long-term water level monitoring in wells at Barnes, January to July 2008. 

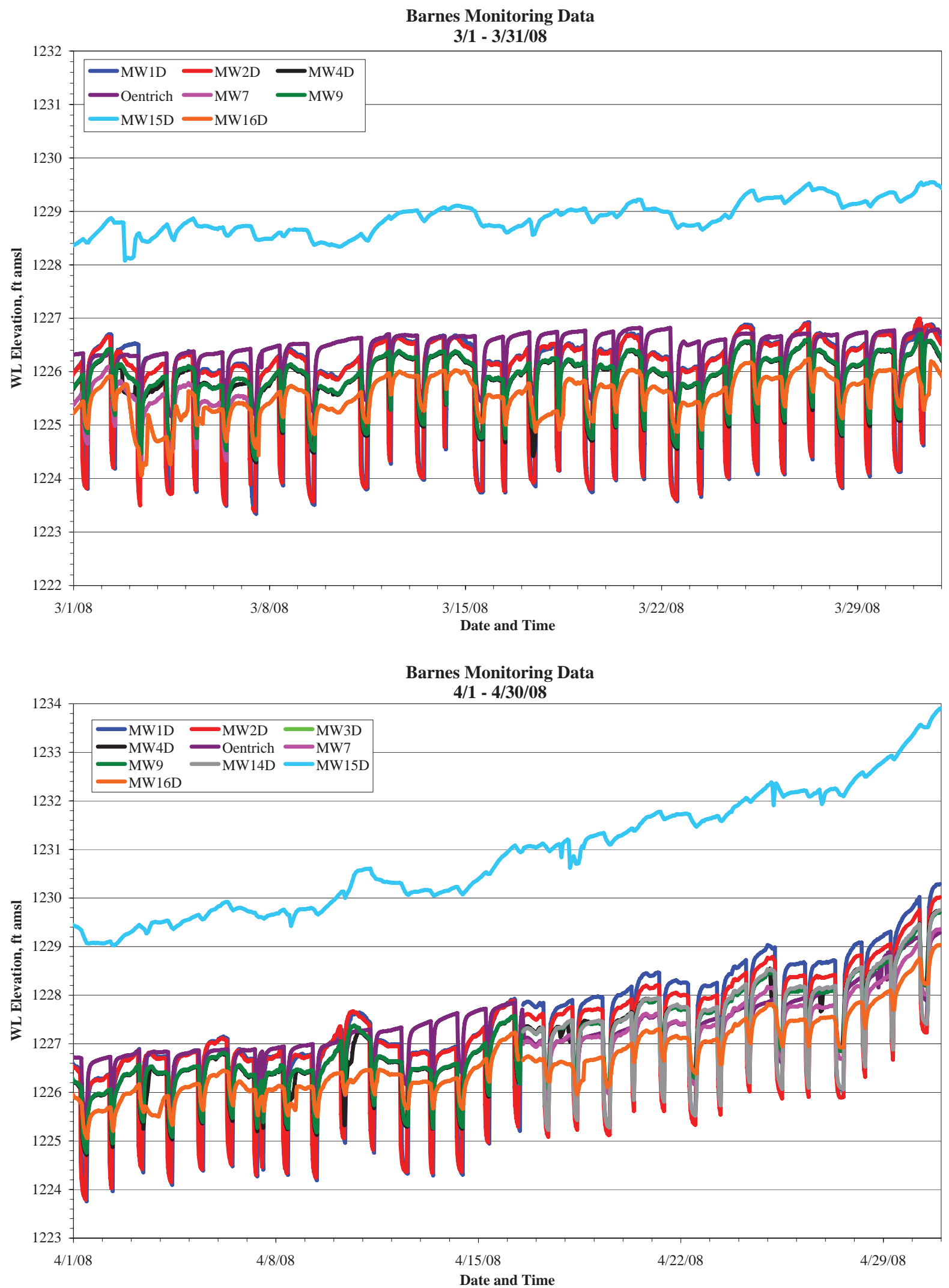

FIGURE 3.2 (Cont.) 

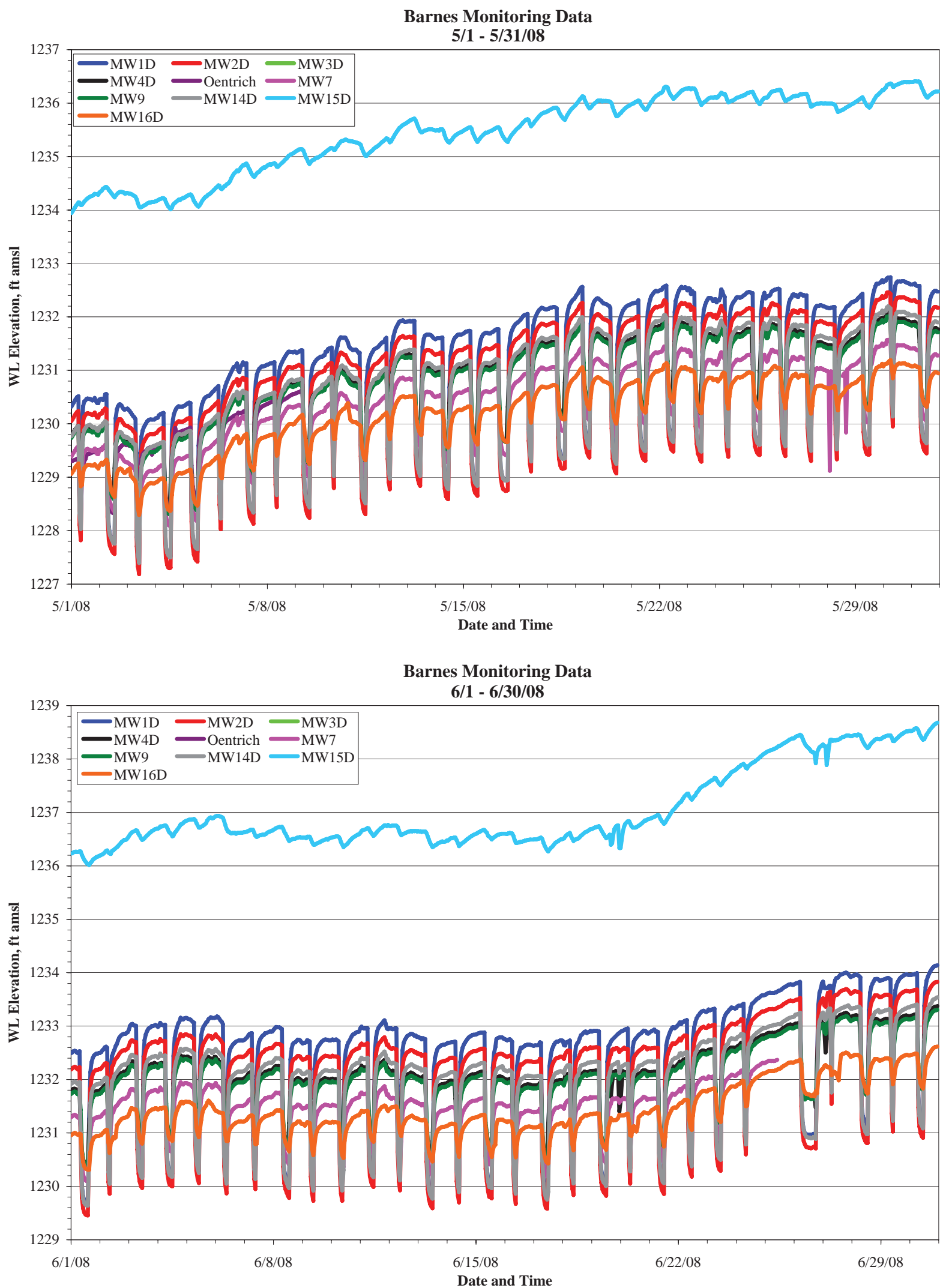

FIGURE 3.2 (Cont.) 


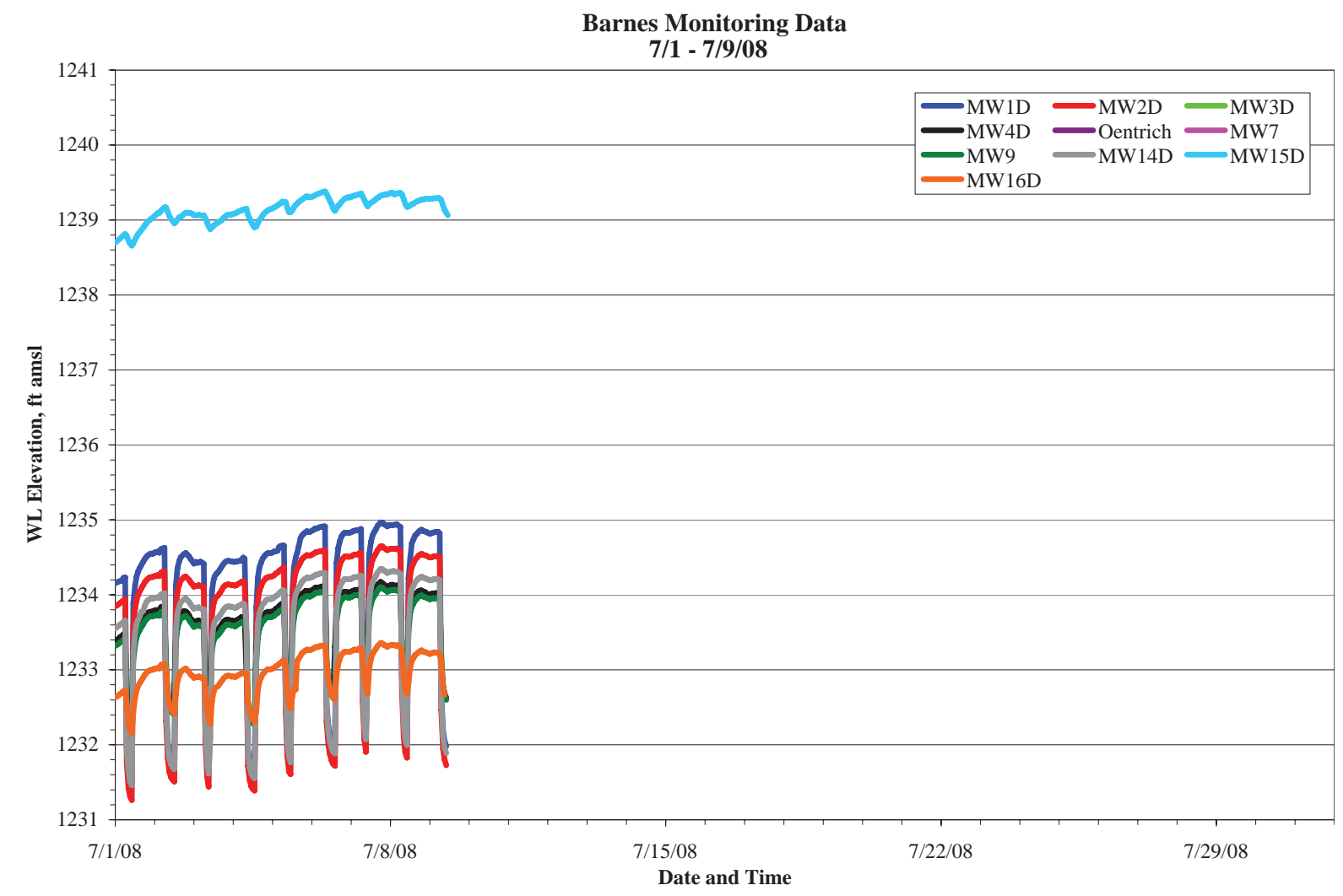

FIGURE 3.2 (Cont.) 


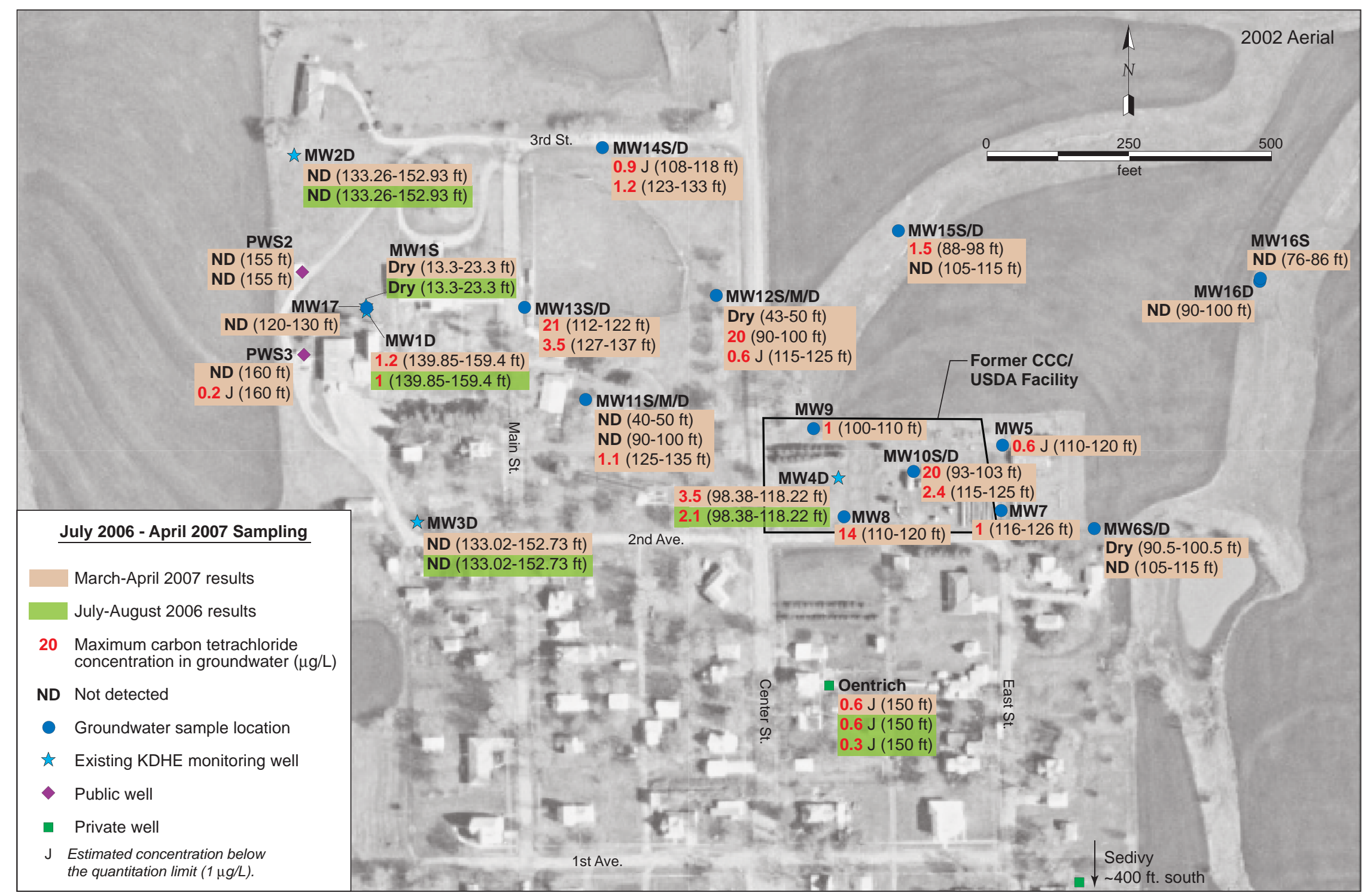

FIGURE 3.3 Analytical results for carbon tetrachloride in groundwater samples collected at Barnes in July-August 2006 and March-April 2007. Source of photograph: NAPP (2002). 


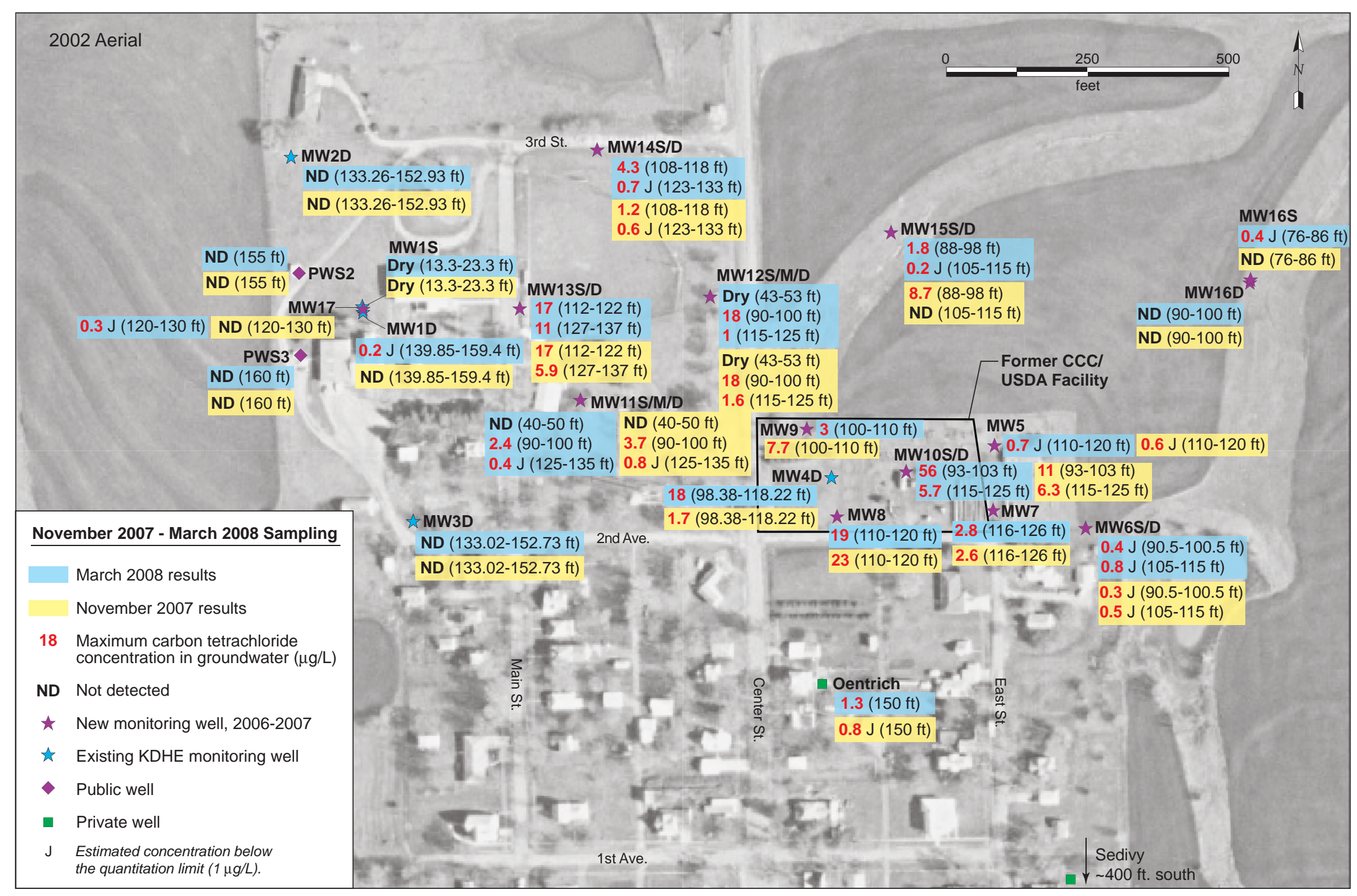

FIGURE 3.4 Analytical results for carbon tetrachloride in groundwater samples collected at Barnes in November 2007 and March 2008. Source of photograph: NAPP (2002). 


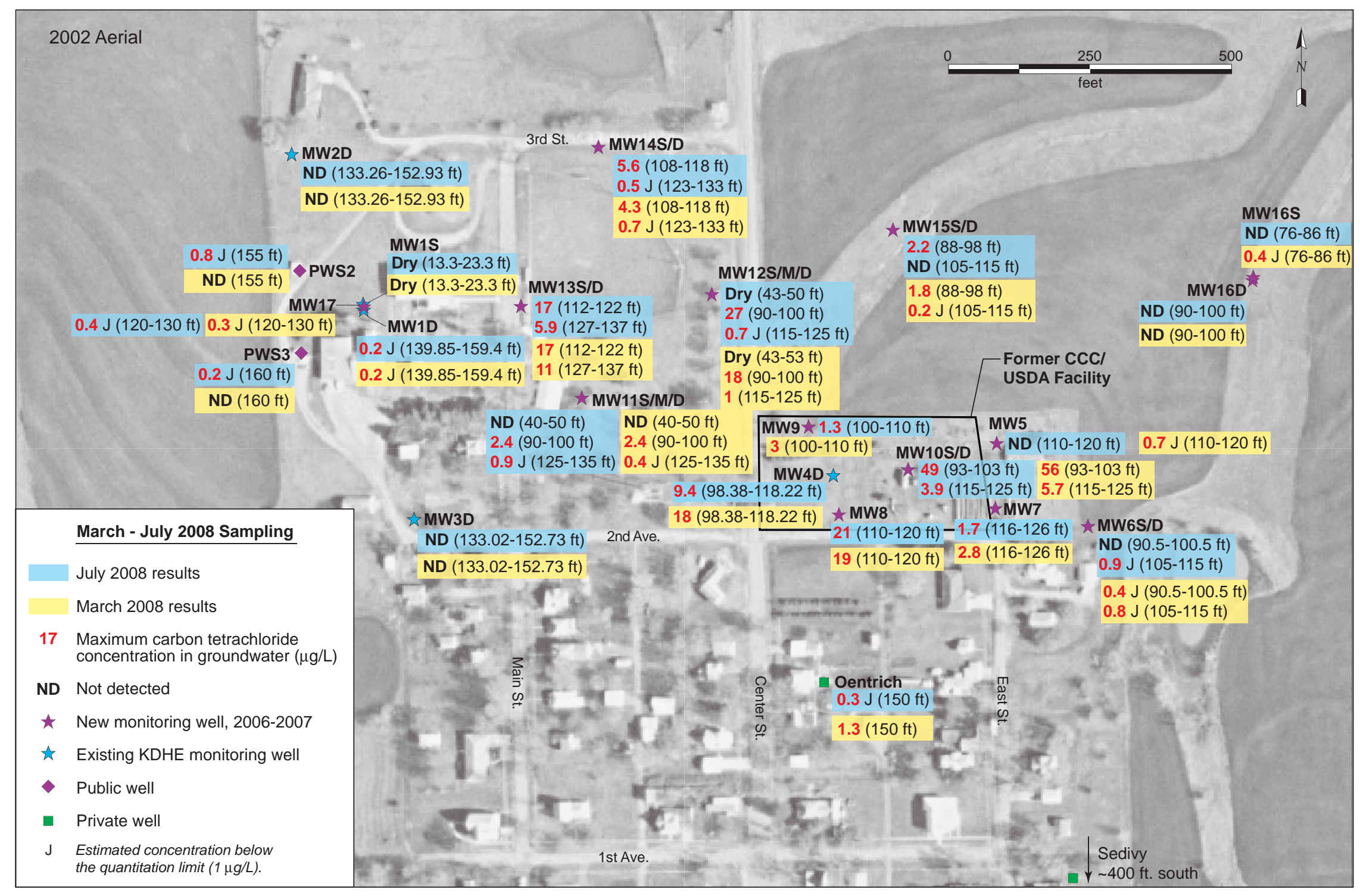

FIGURE 3.5 Analytical results for carbon tetrachloride in groundwater samples collected at Barnes in March 2008 and July 2008 . Source of photograph: NAPP (2002). 


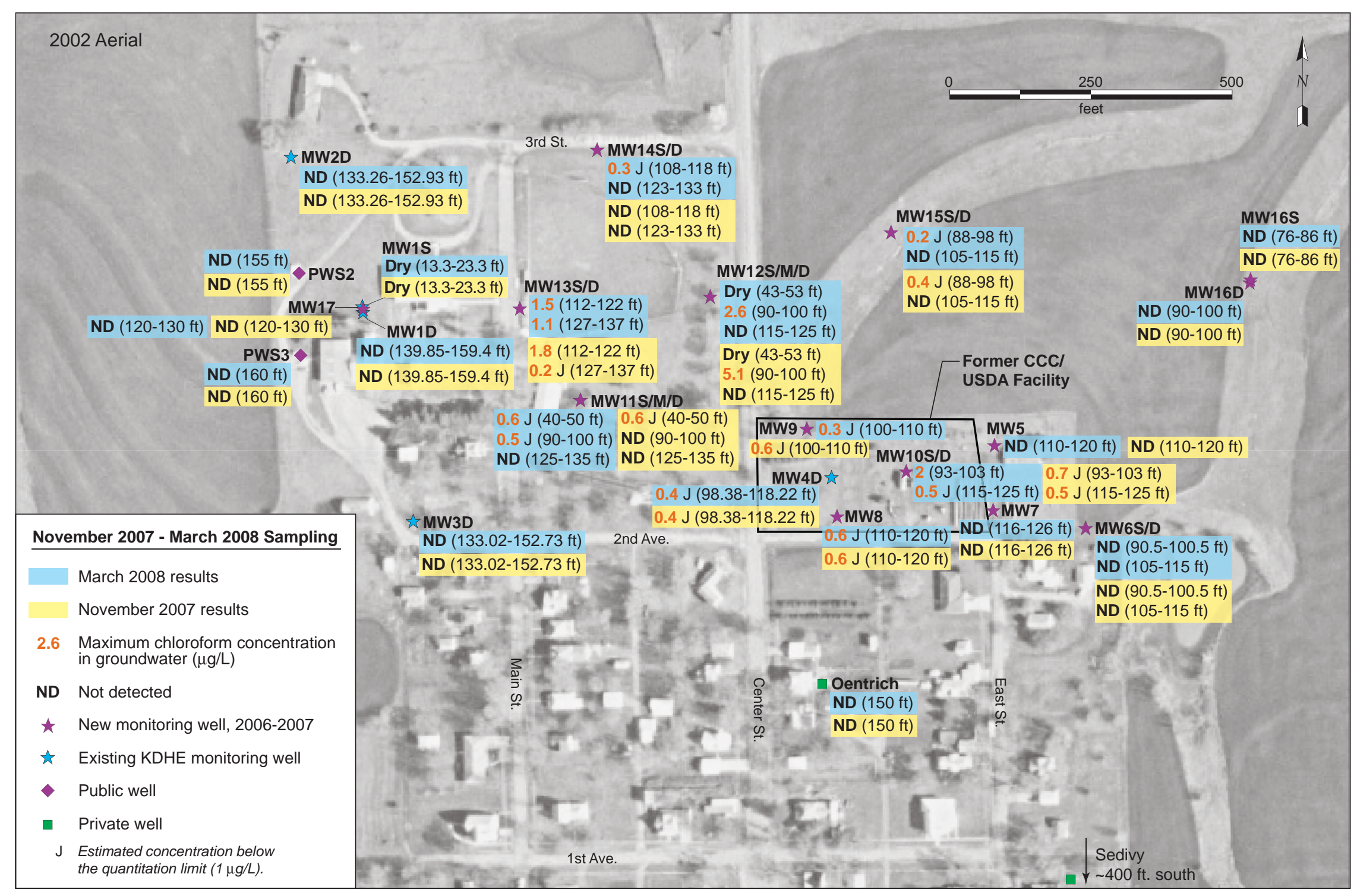

FIGURE 3.6 Analytical results for chloroform in groundwater samples collected at Barnes in November 2007 and March 2008. Source of photograph: NAPP (2002). 


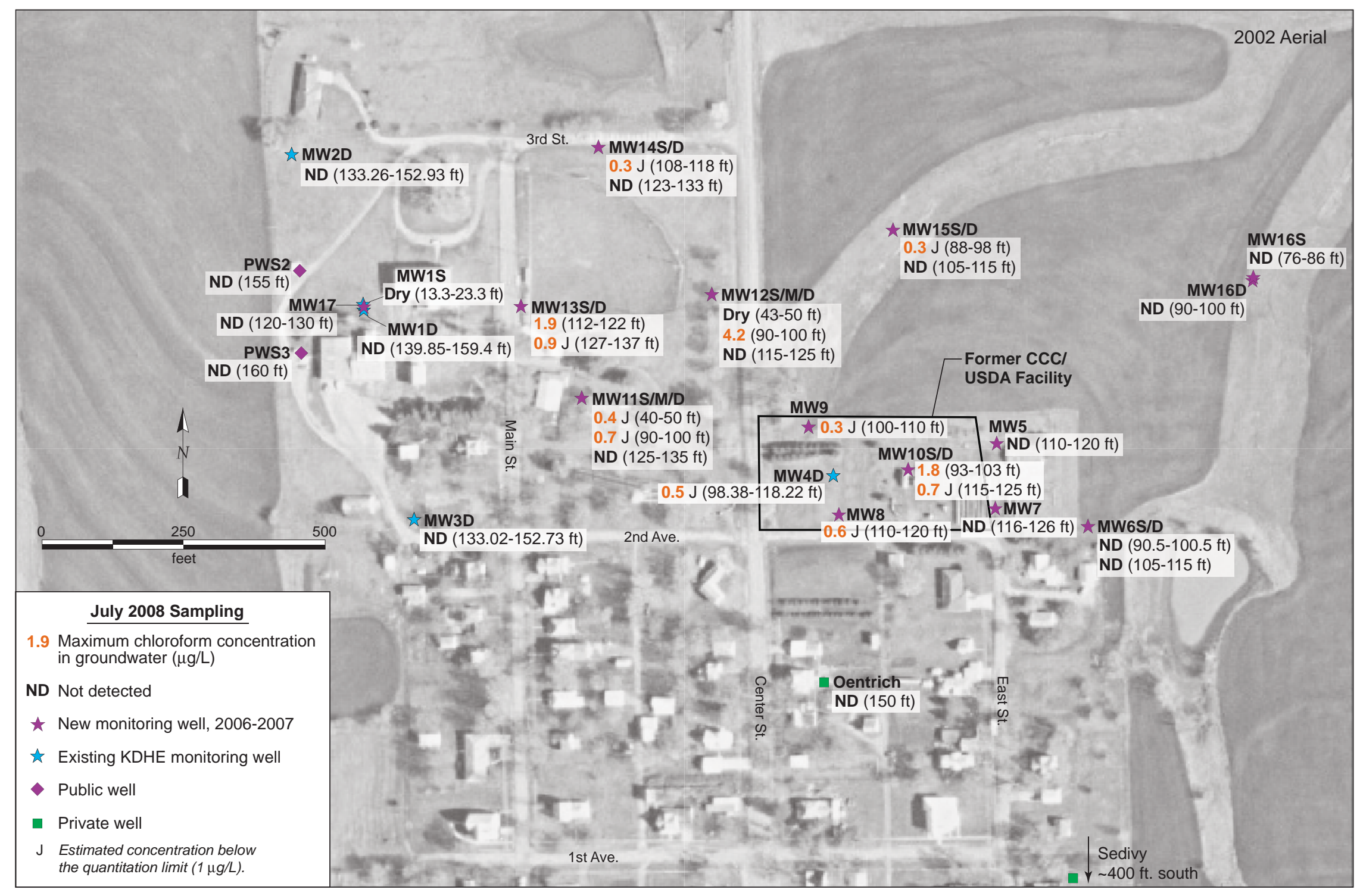

FIGURE 3.7 Analytical results for chloroform in groundwater samples collected at Barnes in July 2008. Source of photograph: NAPP (2002). 


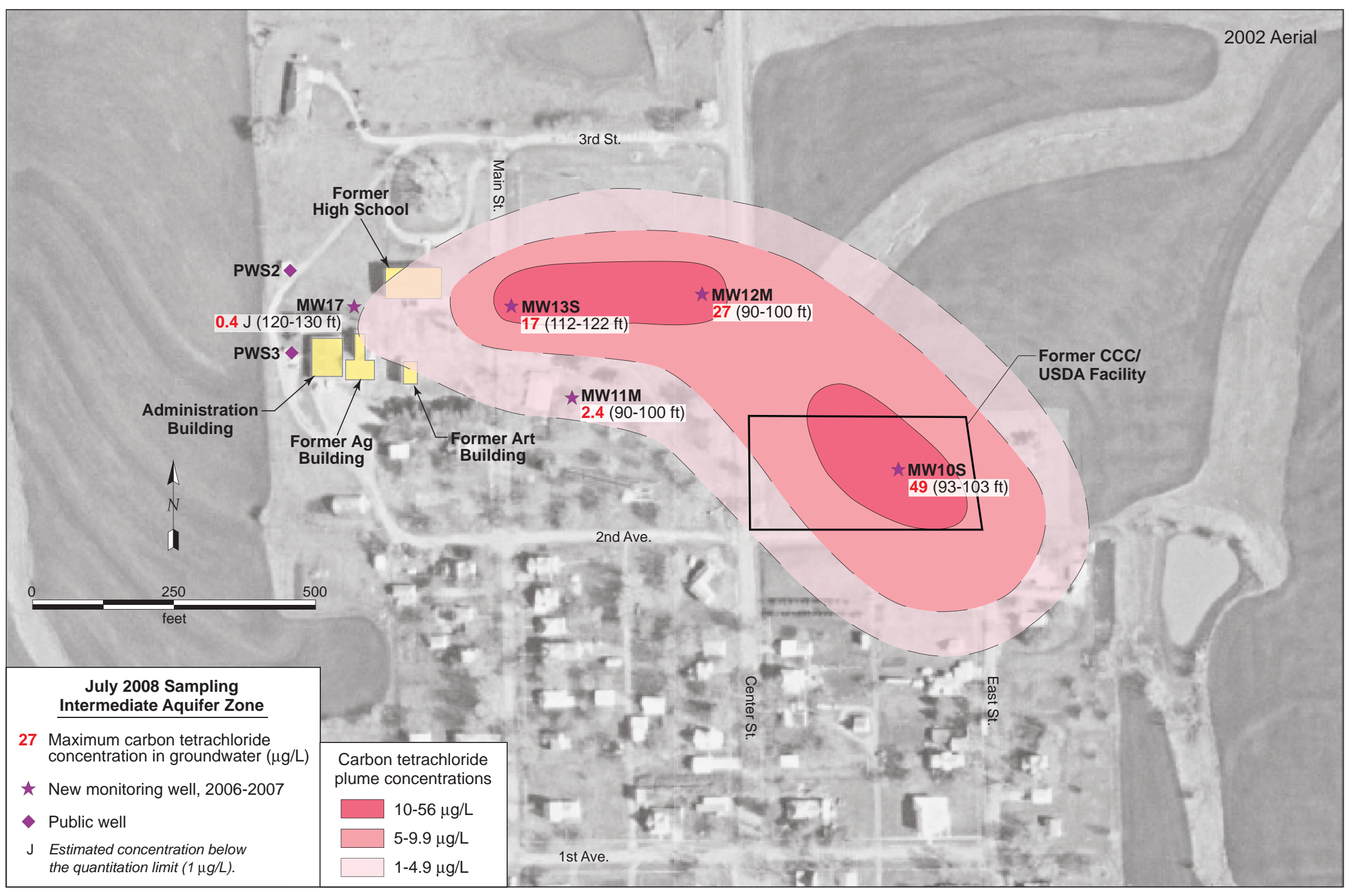

FIGURE 3.8 Interpreted carbon tetrachloride plume in July 2008 in wells screened in the intermediate aquifer zone (approximately 1,255-1,258 ft AMSL). Source of photograph: NAPP (2002). 


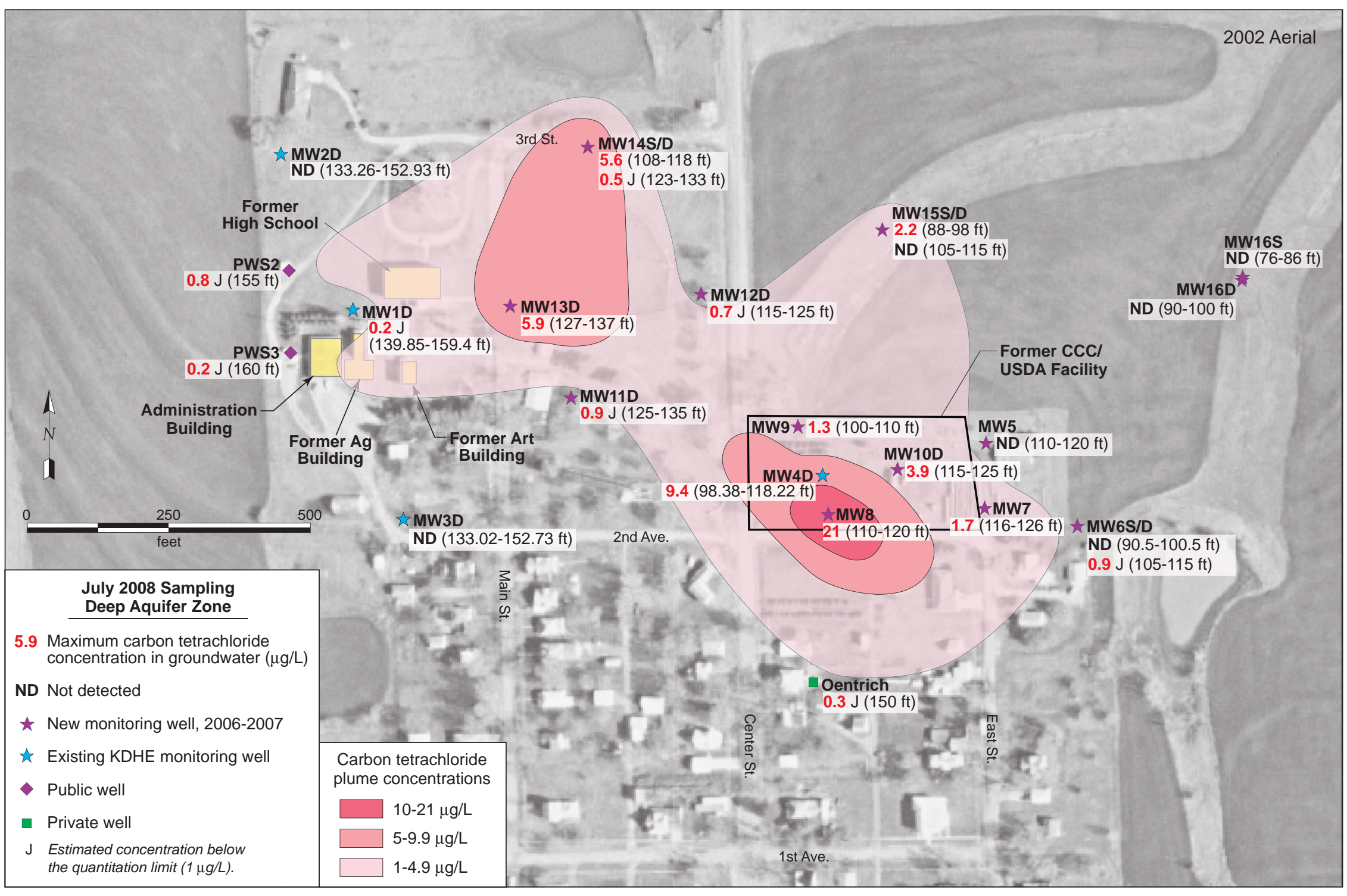

FIGURE 3.9 Interpreted carbon tetrachloride plume in July 2008 in wells screened in the deep aquifer zone (approximately 1,229-1,239 ft AMSL). Source of photograph: NAPP (2002). 


\section{Conclusions and Ongoing Tasks}

\subsection{Conclusions}

The findings of the July 2008 monitoring event at Barnes support the following conclusions:

- Measurements of groundwater levels obtained manually and through the use of automatic recorders have consistently indicated that the flow direction is strongly influenced by pumping of the public water supply wells. The results (in the absence of data for the southern control point, MW3D) indicate

- An apparent groundwater flow direction to the east when the public wells are not pumping and

- A northwesterly groundwater flow trend when the public wells are pumping.

- Overall, the lateral distribution of carbon tetrachloride in groundwater in July 2008 was similar to the distribution during previous sampling events. The most significant change was the trace detections of carbon tetrachloride in public wells PWS2 and PWS3. This marks the first observation since July 2000 (PWS2) or March 2007 (PWS3) of detectable concentrations of carbon tetrachloride in these wells. Protection of these wells is the driving force for the monitoring program at Barnes.

- Analysis of manual water level measurements and carbon tetrachloride concentrations continued to suggest that three vertically distinguishable aquifer zones are present at Barnes: a shallow zone with a potentiometric surface at approximately $1,312 \mathrm{ft}$ AMSL, an intermediate zone at approximately 1,255-1,258 ft AMSL, and a deep zone at approximately 1,229-1,239 ft AMSL. All monitoring wells equipped with data loggers for water level measurements are screened in the deep aquifer zone. 
- Compared to the March 2008 data, the July 2008 data indicate notable decreases in carbon tetrachloride concentrations in two monitoring wells (MW4D and MW13D). Well MW4D is on the former CCC/USDA property, and well MW13D is adjacent to the high school. Both wells are screened in the deep aquifer zone.

- The vertical distribution of the carbon tetrachloride in groundwater continues to indicate that the highest concentrations occurred in the intermediate aquifer zone. Lower concentrations were detected in the deep aquifer zone, and no carbon tetrachloride was detected in the shallow zone.

\subsection{Ongoing Tasks}

Ongoing tasks related to Barnes are as follows:

- Quarterly monitoring will continue, as recommended in the targeted investigation report (Argonne 2008a).

- In an effort to evaluate the effect of pumping of the public water supply wells on the contaminant plume, an attempt will be made to collect the next set of groundwater samples and water level measurements on a schedule coincident with the well pumping schedule.

- In cooperation with the city, daily pumping rates are being recorded over a short time period to provide information for further evaluation of the aquifer characteristics. When this additional information is available, an attempt will be made to model the effects of the pumping, including rates and frequency, on the distribution of carbon tetrachloride in the subsurface. It is anticipated that this effort will include estimation of the capture zone for the pumping public water supply wells.

- The conceptual contingent interim measure work plan requested by the KDHE (2008b) for protection of the public water supply wells is currently being developed. Cost estimates have been obtained for treatment at the wellhead 
through use of carbon filtration or air stripping. The CCC/USDA and Argonne have also contacted Washington County Rural Water District \#2 to determine the feasibility of connecting the city of Barnes to that water supply. 


\section{References}

Argonne, 2002, Final Master Work Plan: Environmental Investigations at Former CCC/USDA Facilities in Kansas, 2002 Revision, ANL/ER/TR-02/004, prepared for the Commodity Credit Corporation, U.S. Department of Agriculture, Washington, D.C., by Argonne National Laboratory, Argonne, Illinois, December.

Argonne, 2008a, Final Report: Results of the 2007-2007 Investigation of Potential Contamination at the Former CCC/USDA facility in Barnes, Kansas, ANL/EVS/AGEM/TR-0712, prepared for the Commodity Credit Corporation, U.S. Department of Agriculture, Washington, D.C., by Argonne National Laboratory, Argonne, Illinois, August.

Argonne, 2008b, November 2007 Monitoring Results for Barnes, Kansas, ANL/EVS/AGEM/TR-08-04, prepared for the Commodity Credit Corporation, U.S. Department of Agriculture, Washington, D.C., by Argonne National Laboratory, Argonne, Illinois, February.

Argonne, 2008c, March 2008 Monitoring Results for Barnes, Kansas, ANL/EVS/AGEM/TR-0811, prepared for the Commodity Credit Corporation, U.S. Department of Agriculture, Washington, D.C., by Argonne National Laboratory, Argonne, Illinois, August.

EPA, 1995, Method 524.2: Measurement of Purgeable Organic Compounds in Water by Capillary Column Gas Chromatography/Mass Spectrometry, Revision 4.1, edited by J.W. Munch, National Exposure Research Laboratory, Office of Research and Development, U.S. Environmental Protection Agency, Cincinnati, Ohio.

KDHE, 2008a, electronic mail message from E. McWilliams (Bureau of Environmental Remediation, Kansas Department of Health and Environment, Topeka, Kansas) to L. Larsen (Larsen and Associates, Inc., Lawrence, Kansas), July 31.

KDHE, 2008b, letter from C. Carey (Bureau of Environmental Remediation, Kansas Department of Health and Environment, Topeka, Kansas) to C. Roe (Commodity Credit Corporation, U.S. Department of Agriculture, Washington, D.C.), regarding the former CCC/USDA facility at Barnes, March 13. 
NAPP, 2002, aerial image NAPP 12897 222, National Aerial Photography Program, U.S. Geological Survey (available from Kansas Geospatial Community Commons [http://www.kansasgis.org/]), April 9.

Puls, R.W., and Barcelona, M.J., 1996, "Low-Flow (Minimal Drawdown) Ground-Water Sampling Procedures," EPA/540/S-95/504, in Ground Water Issue, Superfund Technology Support Center for Ground Water, National Risk Management Research Laboratory, U.S. Environmental Protection Agency, Ada, Oklahoma, April (www.epa.gov/tio/tsp/download/ lwflw2a.pdf). 


\section{Appendix A:}

\section{Sampling Activities and Field Measurements} at Barnes in July 2008 
TABLE A.1 Sequence of sampling activities at Barnes in July 2008. ${ }^{a}$

\begin{tabular}{|c|c|c|c|c|c|c|c|c|}
\hline $\begin{array}{l}\text { Sample } \\
\text { Date }\end{array}$ & Time & Location & Sample & Type & $\begin{array}{l}\text { Depthc } \\
(\mathrm{ft} \text { TOC) }\end{array}$ & $\begin{array}{l}\text { Chain of } \\
\text { Custody }\end{array}$ & $\begin{array}{l}\text { Shipping } \\
\text { Date }\end{array}$ & Sample Description \\
\hline $7 / 9 / 08$ & $12: 00$ & MW1S & - & - & 13.3-23.3 & - & - & Well dry. \\
\hline $7 / 9 / 08$ & $12: 24$ & MW1D & BAMW1D-W-22668 & MW & $139.85-159.4$ & 3579 & $7 / 10 / 08$ & $\begin{array}{l}\text { Depth to water }=119.4 \mathrm{ft} \text { TOC. Depth of } 2 \text {-in. well }= \\
159.4 \mathrm{ft} \text { TOC. Sample collected by using low-flow } \\
\text { bladder pump after purging of } 5 \mathrm{~L} \text {. }\end{array}$ \\
\hline $7 / 9 / 08$ & $13: 46$ & MW17 & BAMW17-W-22695 & MW & $120-130$ & 3579 & $7 / 10 / 08$ & $\begin{array}{l}\text { Depth to water }=96.6 \mathrm{ft} \text { TOC. Depth of } 2 \text {-in. well }= \\
130 \mathrm{t} \text { TOC. Sample collected by using low-flow } \\
\text { bladder pump after purging of } 7 \mathrm{~L} \text {. }\end{array}$ \\
\hline 7/9/08 & $16: 43$ & MW13S & BAMW13S-W-22687 & MW & $112-122$ & 3579 & $7 / 10 / 08$ & $\begin{array}{l}\text { Depth to water }=87 \mathrm{ft} \mathrm{TOC} \text {. Depth of } 2 \text {-in. well }=122 \mathrm{ft} \\
\text { TOC. Sample collected by using low-flow bladder } \\
\text { pump after purging of } 6 \mathrm{~L} \text {. }\end{array}$ \\
\hline $7 / 9 / 08$ & $17: 53$ & MW13D & BAMW13D-W-22688 & MW & $127-137$ & 3579 & $7 / 10 / 08$ & $\begin{array}{l}\text { Depth to water }=107.9 \mathrm{ft} \text { TOC. Depth of } 2 \text {-in. well = } \\
137 \mathrm{ft} \mathrm{TOC.} \mathrm{Sample} \mathrm{collected} \mathrm{by} \mathrm{using} \mathrm{low-flow} \\
\text { bladder pump after purging of } 7 \mathrm{~L} \text {. }\end{array}$ \\
\hline $7 / 10 / 08$ & $10: 50$ & MW3D & BAMW3D-W-22670 & MW & $133.02-152.73$ & 3579 & $7 / 10 / 08$ & $\begin{array}{l}\text { Depth to water }=113.3 \mathrm{ft} \mathrm{TOC} \text {. Depth of } 2 \text {-in. well = } \\
152.73 \mathrm{ft} \text { TOC. Sample collected by using low-flow } \\
\text { bladder pump after purging of } 6 \mathrm{~L} \text {. }\end{array}$ \\
\hline $7 / 10 / 08$ & $11: 38$ & MW2D & BAMW2D-W-22669 & MW & $133.26-152.93$ & 3579 & $7 / 10 / 08$ & $\begin{array}{l}\text { Depth to water }=117.15 \mathrm{ft} \text { TOC. Depth of } 2 \text {-in. well = } \\
152.93 \mathrm{ft} \text { TOC. Sample collected by using low-flow } \\
\text { bladder pump after purging of } 10.5 \mathrm{~L} \text {. }\end{array}$ \\
\hline $7 / 10 / 08$ & $11: 39$ & MW2D & BAMW2DDUP-W-22698 & MW & $133.26-152.93$ & 3579 & $7 / 10 / 08$ & Replicate of sample BAMW2D-W-22669. \\
\hline $7 / 10 / 08$ & $14: 22$ & MW14D & BAMW14D-W-22690 & MW & $123-133$ & 3579 & $7 / 10 / 08$ & $\begin{array}{l}\text { Depth to water = } 101 \mathrm{ft} \mathrm{TOC} \text {. Depth of } 2 \text {-in. well = } \\
133 \mathrm{ftTOC} \text {. Sample collected by using low-flow } \\
\text { bladder pump after purging of } 8.5 \mathrm{~L} \text {. }\end{array}$ \\
\hline $7 / 10 / 08$ & $14: 32$ & MW11S & BAMW11S-W-22681 & MW & $40-50$ & 3579 & $7 / 10 / 08$ & $\begin{array}{l}\text { Depth to water }=24.8 \mathrm{ft} \text { TOC. Depth of } 1 \mathrm{in} \text {. well = } \\
54 \mathrm{ft} \text { TOC. Sample collected by using low-flow } \\
\text { bladder pump after purging of } 3 \mathrm{~L} \text {. }\end{array}$ \\
\hline $7 / 10 / 08$ & $15: 56$ & MW14S & BAMW14S-W-22689 & MW & $108-118$ & 3579 & $7 / 10 / 08$ & $\begin{array}{l}\text { Depth to water }=99.4 \mathrm{ft} \text { TOC. Depth of } 2 \text {-in. well = } \\
118 \mathrm{ft} \text { TOC. Sample collected by using low-flow } \\
\text { bladder pump after purging of } 9 \mathrm{~L} \text {. Aliquots also } \\
\text { collected for verification analysis. }\end{array}$ \\
\hline $7 / 10 / 08$ & $16: 45$ & MW11M & BAMW11M-W-22682 & MW & $90-100$ & 3579 & $7 / 10 / 08$ & $\begin{array}{l}\text { Depth to water }=78.85 \mathrm{ft} \mathrm{TOC} \text {. Depth of } 2 \text {-in. well }= \\
100 \mathrm{ft} \text { TOC. Sample collected by using low-flow } \\
\text { bladder pump after purging of } 6 \mathrm{~L} \text {. }\end{array}$ \\
\hline $7 / 10 / 08$ & $17: 00$ & MW12S & - & - & $43-53$ & - & - & Well dry. \\
\hline $7 / 10 / 08$ & $17: 44$ & MW12M & BAMW12M-W-22685 & MW & $90-100$ & 3579 & $7 / 10 / 08$ & $\begin{array}{l}\text { Depth to water }=70.1 \mathrm{ft} \mathrm{TOC} \text {. Depth of } 2 \text {-in. well = } \\
100 \mathrm{ft} \text { TOC. Sample collected by using low-flow } \\
\text { bladder pump after purging of } 8 \mathrm{~L} \text {. }\end{array}$ \\
\hline
\end{tabular}


TABLE A.1 (Cont.)

\begin{tabular}{|c|c|c|c|c|c|c|c|c|}
\hline $\begin{array}{l}\text { Sample } \\
\text { Date }\end{array}$ & Time & Location & Sample & Type $^{\text {b }}$ & $\begin{array}{c}\text { Depthc } \\
\text { (ft TOC) }\end{array}$ & $\begin{array}{l}\text { Chain of } \\
\text { Custody }\end{array}$ & $\begin{array}{l}\text { Shipping } \\
\text { Date }\end{array}$ & Sample Description \\
\hline 7/10/08 & $18: 21$ & MW11D & BAMW11D-W-22683 & MW & $125-135$ & 3579 & $7 / 10 / 08$ & $\begin{array}{l}\text { Depth to water }=102.1 \mathrm{ft} \mathrm{TOC} \text {. Depth of } 2 \text {-in. well }= \\
135 \mathrm{ft} \text { TOC. Sample collected by using low-flow } \\
\text { bladder pump. }\end{array}$ \\
\hline 7/10/08 & $18: 33$ & QC & BAQCTB-W-22701 & TB & - & 3579 & $7 / 10 / 08$ & $\begin{array}{l}\text { Trip blank sent to the AGEM Laboratory for organic } \\
\text { analyses with water samples listed on chain-of- } \\
\text { custody form (COC) } 3579 \text {. }\end{array}$ \\
\hline 7/11/08 & $8: 56$ & PWS3 & BAPWS3-W-22697 & PW & 160 & 3580 & $7 / 12 / 08$ & $\begin{array}{l}\text { Well was pumped for } 0.5 \mathrm{~h} \text { and then sampled from the } \\
\text { tap in the well shed. }\end{array}$ \\
\hline 7/11/08 & 9:04 & PWS2 & BAPWS2-W-22696 & PW & 155 & 3580 & $7 / 12 / 08$ & $\begin{array}{l}\text { Well was pumped for } 0.5 \mathrm{~h} \text { and then sampled from the } \\
\text { tap in the well shed. }\end{array}$ \\
\hline $7 / 11 / 08$ & $10: 12$ & MW16D & BAMW16D-W-22694 & MW & $90-100$ & 3580 & $7 / 12 / 08$ & $\begin{array}{l}\text { Depth to water }=66.3 \mathrm{ft} \mathrm{TOC} \text {. Depth of } 2 \text {-in. well }= \\
99.6 \mathrm{ft} \text { TOC. Sample collected by using low-flow } \\
\text { bladder pump after purging of } 5.5 \mathrm{~L} \text {. }\end{array}$ \\
\hline $7 / 11 / 08$ & $10: 54$ & MW5 & BAMW5-W-22672 & MW & $110-120$ & 3580 & $7 / 12 / 08$ & $\begin{array}{l}\text { Depth to water }=93.8 \mathrm{ft} \mathrm{TOC} \text {. Depth of } 2 \text {-in. well }= \\
120 \mathrm{ft} \text { TOC. Sample collected by using low-flow } \\
\text { bladder pump after purging of } 8 \mathrm{~L} \text {. }\end{array}$ \\
\hline $7 / 11 / 08$ & $11: 31$ & MW16S & BAMW16S-W-22693 & MW & $76-86$ & 3580 & $7 / 12 / 08$ & $\begin{array}{l}\text { Depth to water }=67.35 \mathrm{ft} \mathrm{TOC} \text {. Depth of } 2 \text {-in. well }= \\
90 \mathrm{ft} \text { TOC. Sample collected by using low-flow } \\
\text { bladder pump after purging of } 10.5 \mathrm{~L} \text {. }\end{array}$ \\
\hline $7 / 11 / 08$ & $12: 10$ & MW6D & BAMW6D-W-22674 & MW & $105-115$ & 3580 & $7 / 12 / 08$ & $\begin{array}{l}\text { Depth to water }=89.5 \mathrm{ft} \text { TOC. Depth of } 2 \text {-in. well }= \\
115 \mathrm{ft} \mathrm{TOC.} \mathrm{Sample} \mathrm{collected} \mathrm{by} \mathrm{using} \mathrm{low-flow} \\
\text { bladder pump after purging of } 5 \mathrm{~L} \text {. }\end{array}$ \\
\hline $7 / 11 / 08$ & 14:09 & MW12D & BAMW12D-W-22686 & MW & $115-125$ & 3580 & $7 / 12 / 08$ & $\begin{array}{l}\text { Depth to water }=93.7 \mathrm{ft} \text { TOC. Depth of } 2 \text {-in. well }= \\
125 \mathrm{ft} \mathrm{TOC.} \mathrm{Sample} \mathrm{collected} \mathrm{by} \mathrm{using} \mathrm{low-flow} \\
\text { bladder pump after purging of } 5.5 \mathrm{~L} \text {. Aliquots also } \\
\text { collected for verification analysis. }\end{array}$ \\
\hline $7 / 11 / 08$ & 14:22 & MW6S & BAMW6S-W-22673 & MW & $90.5-100.5$ & 3580 & $7 / 12 / 08$ & $\begin{array}{l}\text { Depth to water }=88.1 \mathrm{ft} \text { TOC. Depth of } 2 \text {-in. well }= \\
100.5 \mathrm{ft} \mathrm{TOC.} \mathrm{Sample} \mathrm{collected} \mathrm{by} \mathrm{using} \mathrm{low-flow} \\
\text { bladder pump after purging of } 6 \mathrm{~L} \text {. }\end{array}$ \\
\hline $7 / 11 / 08$ & $15: 56$ & MW10S & BAMW10S-W-22679 & MW & $93-103$ & 3580 & 7/12/08 & $\begin{array}{l}\text { Depth to water }=73.4 \mathrm{ft} \text { TOC. Depth of } 2 \text {-in. well }= \\
103 \mathrm{ft} \mathrm{TOC.} \mathrm{Sample} \mathrm{collected} \mathrm{by} \mathrm{using} \mathrm{low-flow} \\
\text { bladder pump after purging of } 6.3 \mathrm{~L} \text {. Aliquots also } \\
\text { collected for verification analysis. }\end{array}$ \\
\hline $7 / 11 / 08$ & 16:04 & QC & BAQCIR-W-22700 & $\mathrm{RI}$ & - & 3580 & $7 / 12 / 08$ & $\begin{array}{l}\text { Rinsate of decontaminated bladder pump after } \\
\text { collection of sample BAMW10S-W-22679. }\end{array}$ \\
\hline
\end{tabular}


TABLE A.1 (Cont.)

\begin{tabular}{|c|c|c|c|c|c|c|c|c|}
\hline $\begin{array}{l}\text { Sample } \\
\text { Date }\end{array}$ & Time & Location & Sample & Type $^{b}$ & $\begin{array}{l}\text { Depthc } \\
\text { (ft TOC) }\end{array}$ & $\begin{array}{l}\text { Chain of } \\
\text { Custody }\end{array}$ & $\begin{array}{l}\text { Shipping } \\
\text { Date }\end{array}$ & Sample Description \\
\hline $7 / 11 / 08$ & $16: 20$ & MW8 & BAMW8-W-22676 & MW & $110-120$ & 3580 & $7 / 12 / 08$ & $\begin{array}{l}\text { Depth to water }=95.75 \mathrm{ft} \text { TOC. Depth of } 2 \text {-in. well = } \\
124 \mathrm{ft} \text { TOC. Sample collected by using low-flow } \\
\text { bladder pump after purging of } 6 \text { L. Aliquots also } \\
\text { collected for verification analysis. }\end{array}$ \\
\hline $7 / 11 / 08$ & $16: 40$ & QC & BAQCIR-W-22701 & $\mathrm{RI}$ & - & 3580 & $7 / 12 / 08$ & $\begin{array}{l}\text { Rinsate of decontaminated bladder pump after } \\
\text { collection of sample BAMW8-W-22676. }\end{array}$ \\
\hline $7 / 11 / 08$ & $17: 58$ & MW10D & BAMW10D-W-22680 & MW & $115-125$ & 3580 & $7 / 12 / 08$ & $\begin{array}{l}\text { Depth to water }=97.3 \mathrm{ft} \text { TOC. Depth of } 2 \text {-in. well }= \\
125 \mathrm{ft} \text { TOC. Sample collected by using low-flow } \\
\text { bladder pump after purging of } 5 \mathrm{~L} \text {. }\end{array}$ \\
\hline $7 / 11 / 08$ & $18: 15$ & Oentrich & BAOENTRICH-W-22695 & DW & 150 & 3580 & $7 / 12 / 08$ & $\begin{array}{l}\text { Hydrant in back yard was allowed to run for } 5 \mathrm{~min} \text {, } \\
\text { then sampled. }\end{array}$ \\
\hline $7 / 11 / 08$ & $18: 17$ & MW9 & BAMW9-W-22678 & MW & $100-110$ & 3580 & $7 / 12 / 08$ & $\begin{array}{l}\text { Depth to water }=87.65 \mathrm{ft} \mathrm{TOC} \text {. Depth of } 2 \text {-in. well = } \\
120 \mathrm{ft} \text { TOC. Sample collected by using low-flow } \\
\text { bladder pump after purging of } 6 \mathrm{~L} \text {. }\end{array}$ \\
\hline $7 / 12 / 08$ & $10: 30$ & MW7 & BAMW7-W-22675 & MW & $116-126$ & 3581 & $7 / 12 / 08$ & $\begin{array}{l}\text { Depth to water }=97.5 \mathrm{ft} \text { TOC. Depth of } 2 \text {-in. well = } \\
126 \mathrm{ft} \text { TOC. Sample collected by using low-flow } \\
\text { bladder pump after purging of } 9.5 \mathrm{~L} \text {. }\end{array}$ \\
\hline $7 / 12 / 08$ & $10: 32$ & MW15D & BAMW15D-W-22692 & MW & $105-115$ & 3581 & $7 / 12 / 08$ & $\begin{array}{l}\text { Depth to water }=70.3 \mathrm{ft} \text { TOC. Depth of } 2 \text {-in. well = } \\
115 \mathrm{ft} \text { TOC. Sample collected by using low-flow } \\
\text { bladder pump after purging of } 7 \mathrm{~L} \text {. }\end{array}$ \\
\hline $7 / 12 / 08$ & $12: 00$ & QC & BAQCTB-W-22702 & TB & - & 3581 & $7 / 12 / 08$ & $\begin{array}{l}\text { Trip blank with sent to the AGEM Laboratory for } \\
\text { organic analyses with water samples listed on } \\
\text { COCs } 3580 \text { and } 3581 \text {; also sent to TestAmerica for } \\
\text { verification organic analysis with samples listed on } \\
\text { COC } 3583 \text {. }\end{array}$ \\
\hline $7 / 12 / 08$ & $12: 02$ & MW15S & BAMW15S-W-22691 & MW & $88-98$ & 3581 & $7 / 12 / 08$ & $\begin{array}{l}\text { Depth to water }=80.3 \mathrm{ft} \text { TOC. Depth of } 2 \text {-in. well = } \\
98 \mathrm{ft} \text { TOC. Sample collected by using low-flow } \\
\text { bladder pump after purging of } 9 \mathrm{~L} .\end{array}$ \\
\hline $7 / 12 / 08$ & $12: 14$ & MW4D & BAMW4D-W-22671 & MW & $\begin{array}{l}98.38- \\
118.22\end{array}$ & 3581 & $7 / 12 / 08$ & $\begin{array}{l}\text { Depth to water }=93.6 \mathrm{ft} \text { TOC. Depth of } 2 \text {-in. well = } \\
118.22 \mathrm{ft} \text { TOC. Sample collected by using low-flow } \\
\text { bladder pump after purging of } 5.5 \mathrm{~L} \text {. }\end{array}$ \\
\hline $7 / 16 / 08$ & $12: 15$ & QC & BNWW & BT & - & Pace & $7 / 16 / 08$ & $\begin{array}{l}\text { Sample of waste purge water generated during the } \\
\text { July } 2008 \text { sampling event. }\end{array}$ \\
\hline $7 / 16 / 08$ & $12: 20$ & QC & BNCNTB & TB & - & Pace & $7 / 16 / 08$ & $\begin{array}{l}\text { Trip blank sent to Pace Analytical Services with } \\
\text { wastewater sample for VOCs, ethylene dibromide, } \\
\text { and nitrate analyses. }\end{array}$ \\
\hline
\end{tabular}


TABLE A.1 (Cont.)

a All samples collected were water.

b Sample types: BT, wastewater; DW, domestic well; MW, monitoring well; PW, public water supply well; RI, rinsate; TB, trip blank

c Depth is in feet below the top of the well casing.

d NR, not recorded. 
TABLE A.2 Field measurements for groundwater samples collected at Barnes, July 2006 to July 2008 .

\begin{tabular}{|c|c|c|c|c|c|c|c|}
\hline Well & $\begin{array}{l}\text { Screen Interval } \\
\quad(\mathrm{ft} B G L)\end{array}$ & $\begin{array}{l}\text { Sample } \\
\text { Date }\end{array}$ & $\begin{array}{c}\text { Temperature } \\
\left({ }^{\circ} \mathrm{C}\right)\end{array}$ & $\mathrm{pH}$ & $\begin{array}{l}\text { Conductivity } \\
(\mu \mathrm{S} / \mathrm{cm})\end{array}$ & $\begin{array}{c}\text { Dissolved } \\
\text { Oxygen } \\
(\mathrm{mg} / \mathrm{L})\end{array}$ & $\begin{array}{c}\text { Oxidation } \\
\text { Reduction } \\
\text { Potential } \\
(\mathrm{mV})\end{array}$ \\
\hline \multicolumn{8}{|c|}{ Previously existing KDHE monitoring wells } \\
\hline \multirow[t]{5}{*}{ MW1S } & 13.3-23.3 & $7 / 19 / 06^{a}$ & - & - & - & - & - \\
\hline & & $4 / 4 / 07^{a}$ & - & - & - & - & - \\
\hline & & $11 / 18 / 07^{a}$ & - & - & - & - & - \\
\hline & & $3 / 4 / 08^{a}$ & - & - & - & - & - \\
\hline & & $7 / 9 / 08^{a}$ & - & - & - & - & - \\
\hline \multirow[t]{5}{*}{ MW1D } & $139.85-159.4$ & $7 / 19 / 06$ & 22.8 & 7.15 & 945 & - & - \\
\hline & & $4 / 4 / 07$ & 15.7 & 6.30 & 855 & - & - \\
\hline & & $11 / 18 / 07$ & 12.7 & 7.62 & 712 & - & - \\
\hline & & $3 / 4 / 08$ & 5.5 & 7.22 & 1167 & 11.6 & 244.2 \\
\hline & & $7 / 9 / 08$ & 18.0 & 7.05 & 992 & 16.2 & 97.5 \\
\hline \multirow[t]{5}{*}{ MW2D } & $133.26-152.93$ & $7 / 19 / 06$ & 24.7 & 7.72 & 946 & - & - \\
\hline & & $4 / 4 / 07$ & 15.1 & 6.32 & 887 & - & - \\
\hline & & $11 / 18 / 07$ & 12.1 & 6.96 & 1448 & - & - \\
\hline & & $3 / 7 / 08$ & 6.5 & 7.22 & 1198 & 4.61 & 196.5 \\
\hline & & $7 / 10 / 08$ & 18.4 & 6.91 & 1163 & 5.03 & 155.3 \\
\hline \multirow[t]{4}{*}{ MW3D } & $133.02-152.73$ & $7 / 19 / 06$ & 23.0 & 7.06 & 976 & - & - \\
\hline & & $4 / 4 / 07$ & 15.6 & 6.37 & 989 & - & - \\
\hline & & $11 / 19 / 07$ & 10.5 & 7.16 & 1093 & - & - \\
\hline & & $\begin{array}{r}3 / 7 / 08 \\
7 / 10 / 08\end{array}$ & $\begin{array}{r}8.2 \\
19.8\end{array}$ & $\begin{array}{l}7.09 \\
6.99\end{array}$ & $\begin{array}{l}1195 \\
1177\end{array}$ & $\begin{array}{l}5.34 \\
13.8\end{array}$ & $\begin{array}{l}254.8 \\
109.9\end{array}$ \\
\hline \multirow[t]{5}{*}{ MW4D } & $98.38-118.22$ & $7 / 20 / 06$ & 23.5 & 6.26 & 968 & - & - \\
\hline & & $4 / 6 / 07$ & 11.3 & 6.21 & 1018 & - & - \\
\hline & & $11 / 19 / 07$ & 15.7 & 6.98 & 1022 & - & - \\
\hline & & $3 / 9 / 08$ & 11.5 & 7.14 & 859 & 6.57 & 201.2 \\
\hline & & $7 / 12 / 08$ & 14.4 & 6.94 & 1001 & 6.77 & 148.7 \\
\hline
\end{tabular}

CCC/USDA wells installed during 2006-2007 investigation

\begin{tabular}{|c|c|c|c|c|c|c|c|}
\hline \multirow[t]{4}{*}{ MW5 } & \multirow[t]{4}{*}{$110-120$} & $4 / 6 / 07$ & 13.9 & 6.17 & 1705 & - & - \\
\hline & & $11 / 19 / 07$ & 15.2 & 6.74 & 3070 & - & - \\
\hline & & $3 / 8 / 08$ & 9.9 & 6.76 & 2770 & 0.66 & 123.2 \\
\hline & & $7 / 11 / 08$ & 18.8 & 6.66 & 2930 & 1.32 & 36.6 \\
\hline
\end{tabular}


TABLE A.2 (Cont.)

\begin{tabular}{|c|c|c|c|c|c|c|c|}
\hline Well & $\begin{array}{l}\text { Screen Interval } \\
\quad(\mathrm{ft} B G L)\end{array}$ & $\begin{array}{l}\text { Sample } \\
\text { Date }\end{array}$ & $\begin{array}{c}\text { Temperature } \\
\left({ }^{\circ} \mathrm{C}\right)\end{array}$ & $\mathrm{pH}$ & $\begin{array}{c}\text { Conductivity } \\
(\mu \mathrm{S} / \mathrm{cm})\end{array}$ & $\begin{array}{c}\text { Dissolved } \\
\text { Oxygen } \\
(\mathrm{mg} / \mathrm{L})\end{array}$ & $\begin{array}{c}\text { Oxidatior } \\
\text { Reductio } \\
\text { Potentia } \\
(\mathrm{mV})\end{array}$ \\
\hline \multicolumn{8}{|c|}{ CCC/USDA wells installed during 2006-2007 investigation (cont.) } \\
\hline \multirow[t]{4}{*}{ MW6S } & $90.5-100.5$ & $4 / 4 / 07^{a}$ & - & - & - & - & - \\
\hline & & $11 / 19 / 07$ & 12.0 & 7.60 & 723 & - & - \\
\hline & & 3/8/08 & 4.7 & 7.77 & 673 & 6.72 & 272.2 \\
\hline & & $7 / 11 / 08$ & 28.2 & 7.61 & 753 & 9.85 & 92.4 \\
\hline \multirow[t]{4}{*}{ MW6D } & $105-115$ & $4 / 5 / 07$ & 6.2 & 6.11 & 936 & - & - \\
\hline & & $11 / 19 / 07$ & 13.6 & 7.00 & 1103 & - & - \\
\hline & & $3 / 8 / 08$ & 9.1 & 7.15 & 908 & 5.56 & 241.0 \\
\hline & & $7 / 11 / 08$ & 19.8 & 7.05 & 999 & 12.8 & 99.9 \\
\hline \multirow[t]{4}{*}{ MW7 } & $116-126$ & $4 / 6 / 07$ & 14.1 & 6.30 & 1051 & - & - \\
\hline & & $11 / 19 / 07$ & 14.6 & 7.16 & 890 & - & - \\
\hline & & $3 / 9 / 08$ & 13.1 & 7.10 & 1068 & 4.24 & 186.4 \\
\hline & & $7 / 12 / 08$ & 14.4 & 6.95 & 1238 & 4.36 & 98.3 \\
\hline \multirow[t]{4}{*}{ MW8 } & $110-120$ & $4 / 6 / 07$ & 12.1 & 6.23 & 974 & - & - \\
\hline & & $11 / 19 / 07$ & 14.6 & 7.03 & 909 & - & - \\
\hline & & 3/10/08 & 13.1 & 7.09 & 961 & 6.71 & 182.1 \\
\hline & & $7 / 11 / 08$ & 18.6 & 6.38 & 1049 & 6.19 & 152.2 \\
\hline \multirow[t]{4}{*}{ MW9 } & $100-110$ & $4 / 5 / 07$ & 12.9 & 6.20 & 976 & - & - \\
\hline & & $11 / 19 / 07$ & 16.5 & 7.21 & 1066 & - & - \\
\hline & & $3 / 9 / 08$ & 11.2 & 7.07 & 928 & 5.80 & 239.0 \\
\hline & & $7 / 11 / 08$ & 17.7 & 6.58 & 1010 & 5.63 & 188.7 \\
\hline \multirow[t]{4}{*}{ MW10S } & $93-103$ & $4 / 6 / 07$ & 13.2 & 6.36 & 1004 & - & - \\
\hline & & $11 / 19 / 07$ & 14.5 & 7.22 & 942 & - & - \\
\hline & & 3/10/08 & 12.7 & 7.08 & 912 & 5.18 & 176.1 \\
\hline & & $7 / 11 / 08$ & 17.3 & 6.91 & 975 & 12.8 & 118.6 \\
\hline \multirow[t]{4}{*}{ MW10D } & $115-125$ & $4 / 6 / 07$ & 12.1 & 6.21 & 992 & - & - \\
\hline & & $11 / 19 / 07$ & 14.5 & 7.42 & 1175 & - & - \\
\hline & & $3 / 9 / 08$ & 13.7 & 7.01 & 1024 & 5.07 & 235.9 \\
\hline & & $7 / 11 / 08$ & 17.4 & 6.78 & 1090 & 12.6 & 117.1 \\
\hline \multirow[t]{4}{*}{ MW11S } & $40-50$ & $4 / 4 / 07$ & 12.8 & 6.14 & 1027 & - & - \\
\hline & & $11 / 19 / 07$ & 11.2 & 7.15 & 1174 & - & - \\
\hline & & $3 / 5 / 08$ & 9.4 & 6.81 & 1122 & 2.26 & 240.8 \\
\hline & & $7 / 10 / 08$ & 19.5 & 6.47 & 1224 & 1.86 & 166.2 \\
\hline
\end{tabular}


TABLE A.2 (Cont.)

\begin{tabular}{|c|c|c|c|c|c|c|c|}
\hline Well & $\begin{array}{l}\text { Screen Interval } \\
\quad(\mathrm{ft} B G L)\end{array}$ & $\begin{array}{l}\text { Sample } \\
\text { Date }\end{array}$ & $\begin{array}{c}\text { Temperature } \\
\left({ }^{\circ} \mathrm{C}\right)\end{array}$ & $\mathrm{pH}$ & $\begin{array}{l}\text { Conductivity } \\
(\mu \mathrm{S} / \mathrm{cm})\end{array}$ & $\begin{array}{c}\text { Dissolved } \\
\text { Oxygen } \\
(\mathrm{mg} / \mathrm{L})\end{array}$ & $\begin{array}{c}\text { Oxidation } \\
\text { Reduction } \\
\text { Potential } \\
\quad(\mathrm{mV})\end{array}$ \\
\hline \multicolumn{8}{|c|}{ CCC/USDA wells installed during 2006-2007 investigation (cont.) } \\
\hline \multirow[t]{4}{*}{ MW11M } & $90-100$ & $4 / 5 / 07$ & 7.5 & 7.60 & 1097 & - & - \\
\hline & & $11 / 19 / 07$ & 11.9 & 7.17 & 1144 & - & - \\
\hline & & $3 / 6 / 08$ & 10.8 & 7.06 & 997 & 2.65 & 254.1 \\
\hline & & $7 / 10 / 08$ & 31.9 & 7.08 & 1124 & 3.88 & 148.9 \\
\hline \multirow[t]{4}{*}{ MW11D } & $125-135$ & $4 / 4 / 07$ & 13.8 & 6.18 & 990 & - & - \\
\hline & & $11 / 19 / 07$ & 13.1 & 7.22 & 987 & - & - \\
\hline & & $3 / 5 / 08$ & 6.04 & 7.06 & 872 & 6.85 & 252.0 \\
\hline & & $7 / 10 / 08$ & 17.5 & 6.25 & 957 & 7.14 & 176.6 \\
\hline \multirow[t]{4}{*}{ MW12S } & $43-53$ & $4 / 5 / 07^{a}$ & - & - & - & - & - \\
\hline & & $11 / 19 / 07^{a}$ & - & - & - & - & - \\
\hline & & $3 / 10 / 08^{a}$ & - & - & - & - & - \\
\hline & & $7 / 10 / 08^{a}$ & - & - & - & - & - \\
\hline \multirow[t]{4}{*}{ MW12M } & $90-100$ & $4 / 5 / 07$ & 12.6 & 6.42 & 867 & - & - \\
\hline & & $11 / 19 / 07$ & 14.9 & 7.13 & 835 & - & - \\
\hline & & 3/10/08 & 12.6 & 7.13 & 665 & 1.81 & 211.5 \\
\hline & & 7/10/08 & 16.9 & 7.09 & 878 & 8.17 & 87.1 \\
\hline \multirow[t]{4}{*}{ MW12D } & $115-125$ & $4 / 5 / 07$ & 14 & 6.36 & 930 & - & - \\
\hline & & $11 / 18 / 07$ & 15.6 & 6.95 & 571 & - & - \\
\hline & & $3 / 9 / 08$ & 8.8 & 7.13 & 881 & 5.25 & 237.3 \\
\hline & & $7 / 11 / 08$ & 19.9 & 6.01 & 987 & 4.72 & 196.5 \\
\hline \multirow[t]{4}{*}{ MW13S } & $112-122$ & $4 / 5 / 07$ & 9.8 & 6.42 & 946 & - & - \\
\hline & & $11 / 19 / 07$ & 16.5 & 7.21 & 893 & - & - \\
\hline & & 3/10/08 & 12.2 & 7.13 & 810 & 6.21 & 199.3 \\
\hline & & 7/9/08 & 17.4 & 6.99 & 875 & 7.72 & 115.7 \\
\hline \multirow[t]{4}{*}{ MW13D } & $127-137$ & $4 / 5 / 07$ & 14.9 & 6.25 & 397 & - & - \\
\hline & & $11 / 19 / 07$ & 17 & 7.00 & 763 & - & - \\
\hline & & $3 / 9 / 08$ & 13.1 & 7.09 & 758 & 5.95 & 212.9 \\
\hline & & $7 / 9 / 08$ & 18.6 & 7.07 & 848 & 18.1 & 56.9 \\
\hline \multirow[t]{4}{*}{ MW14S } & 108-118 & $4 / 4 / 07$ & 13.4 & 6.50 & 704 & - & - \\
\hline & & $11 / 18 / 07$ & 12.9 & 7.26 & 966 & - & - \\
\hline & & $3 / 8 / 08$ & 13.2 & 7.20 & 729 & 6.59 & 207.8 \\
\hline & & $7 / 10 / 08$ & 17.4 & 7.16 & 775 & 16.4 & 86.7 \\
\hline
\end{tabular}


TABLE A.2 (Cont.)

\begin{tabular}{|c|c|c|c|c|c|c|c|}
\hline Well & $\begin{array}{l}\text { Screen Interval } \\
\quad(\mathrm{ft} \mathrm{BGL})\end{array}$ & $\begin{array}{l}\text { Sample } \\
\text { Date }\end{array}$ & $\begin{array}{c}\text { Temperature } \\
\left({ }^{\circ} \mathrm{C}\right)\end{array}$ & $\mathrm{pH}$ & $\begin{array}{l}\text { Conductivity } \\
(\mu \mathrm{S} / \mathrm{cm})\end{array}$ & $\begin{array}{c}\text { Dissolved } \\
\text { Oxygen } \\
\text { (mg/L) }\end{array}$ & $\begin{array}{c}\text { Oxidation } \\
\text { Reduction } \\
\text { Potential } \\
(\mathrm{mV})\end{array}$ \\
\hline \multicolumn{8}{|c|}{ CCC/USDA wells installed during 2006-2007 investigation (cont.) } \\
\hline \multirow[t]{4}{*}{ MW14D } & $123-133$ & $4 / 4 / 07$ & 14.7 & 6.34 & 932 & - & - \\
\hline & & $11 / 18 / 07$ & 13.2 & 7.47 & 739 & - & - \\
\hline & & $3 / 8 / 08$ & 12.0 & 7.06 & 1424 & 1.95 & 282.4 \\
\hline & & $7 / 10 / 08$ & 17.7 & 7.07 & 1459 & 14.4 & 85.7 \\
\hline \multirow[t]{4}{*}{ MW15S } & $88-98$ & $4 / 4 / 07$ & 13.1 & 8.03 & 854 & - & - \\
\hline & & $11 / 18 / 07$ & 13.9 & - & 1883 & - & - \\
\hline & & $3 / 10 / 08$ & 12.1 & 8.67 & 697 & 5.49 & 173.2 \\
\hline & & $7 / 12 / 08$ & 14.0 & 8.88 & 660 & 11.6 & 93.7 \\
\hline \multirow[t]{4}{*}{ MW15D } & $105-115$ & $4 / 4 / 07$ & 14.8 & 6.15 & 2980 & - & - \\
\hline & & $11 / 18 / 07$ & 13.1 & 6.85 & 2190 & - & - \\
\hline & & $3 / 8 / 08$ & 9.0 & 6.85 & 2912 & 0.57 & 130.6 \\
\hline & & $7 / 12 / 08$ & 14.1 & 6.80 & 3067 & 1.05 & 89.6 \\
\hline \multirow[t]{4}{*}{ MW16S } & $76-86$ & $4 / 4 / 07$ & 12.8 & 6.35 & 1708 & - & - \\
\hline & & $11 / 19 / 07$ & 15.0 & 6.94 & 1616 & - & - \\
\hline & & $3 / 7 / 08$ & 7.3 & 6.96 & 1968 & 3.45 & 183.5 \\
\hline & & $7 / 11 / 08$ & 18.8 & 6.71 & 2883 & 1.13 & 52.0 \\
\hline \multirow[t]{4}{*}{ MW16D } & $90-100$ & $4 / 4 / 07$ & 14.1 & 6.17 & 2910 & - & - \\
\hline & & $11 / 19 / 07$ & 12.5 & 6.78 & 2400 & - & - \\
\hline & & $3 / 7 / 08$ & 7.0 & 6.86 & 2866 & 0.5 & 140.0 \\
\hline & & $7 / 11 / 08$ & 18.9 & 6.64 & 3134 & 0.41 & 31.5 \\
\hline \multirow[t]{4}{*}{ MW17 } & $120-130$ & $4 / 4 / 07$ & 16.0 & 6.44 & 861 & - & - \\
\hline & & $11 / 19 / 07$ & 8.3 & 7.15 & 610 & - & - \\
\hline & & $3 / 5 / 08$ & 5.4 & 7.12 & 804 & 7.02 & 239.4 \\
\hline & & $7 / 9 / 08$ & 17.5 & 7.11 & 843 & 20.6 & 89.4 \\
\hline
\end{tabular}

Private wells

Oentrich

150

$\begin{array}{rclr}7 / 20 / 06 & - & - & - \\ 8 / 2 / 06 & - & - & - \\ 4 / 5 / 07 & - & - & - \\ 11 / 19 / 07 & 12.1 & 8.26 & 1830 \\ 3 / 6 / 08 & - & - & - \\ 7 / 11 / 08 & - & - & -\end{array}$


TABLE A.2 (Cont.)

\begin{tabular}{|c|c|c|c|c|c|c|c|}
\hline Well & $\begin{array}{l}\text { Screen Interval } \\
\quad(\mathrm{ft} B G \mathrm{~L})\end{array}$ & $\begin{array}{l}\text { Sample } \\
\text { Date }\end{array}$ & $\begin{array}{c}\text { Temperature } \\
\left({ }^{\circ} \mathrm{C}\right)\end{array}$ & $\mathrm{pH}$ & $\begin{array}{l}\text { Conductivity } \\
(\mu \mathrm{S} / \mathrm{cm})\end{array}$ & $\begin{array}{c}\text { Dissolved } \\
\text { Oxygen } \\
(\mathrm{mg} / \mathrm{L})\end{array}$ & $\begin{array}{c}\text { Oxidation } \\
\text { Reduction } \\
\text { Potential } \\
\text { (mV) }\end{array}$ \\
\hline \multicolumn{8}{|c|}{ Private wells (cont.) } \\
\hline \multirow[t]{2}{*}{ Sedivy } & 138 & $8 / 22 / 06$ & - & - & - & - & - \\
\hline & & $9 / 13 / 06$ & 22.5 & 6.57 & 739 & - & - \\
\hline
\end{tabular}

a Not sampled; well dry. 


\section{Appendix B:}

\section{Results from the AGEM Laboratory for Dual Analyses} of Samples Collected at Barnes in July 2008 and for Quality Control Samples 
TABLE B.1 Analytical results for samples and replicates collected at Barnes in July 2008 and for quality control samples.

\begin{tabular}{|c|c|c|c|c|c|c|c|}
\hline \multirow[b]{2}{*}{ Location } & \multirow[b]{2}{*}{ Sample } & \multirow[b]{2}{*}{$\begin{array}{c}\text { Sample } \\
\text { Date }\end{array}$} & \multirow[b]{2}{*}{$\begin{array}{c}\text { Depth } \\
\text { (ft BGL) }\end{array}$} & \multicolumn{3}{|c|}{ Concentration $(\mu \mathrm{g} / \mathrm{L})$} & \multirow[b]{2}{*}{$\begin{array}{c}\text { Analysis } \\
\text { Type }\end{array}$} \\
\hline & & & & $\begin{array}{c}\text { Carbon } \\
\text { Tetrachloride }\end{array}$ & Chloroform & $\begin{array}{l}\text { Methylene } \\
\text { Chloride }\end{array}$ & \\
\hline MW2D & BAMW2D-W-22669 & $7 / 10 / 08$ & $133.26-152.93$ & $N D^{a}$ & ND & ND & Primary \\
\hline MW2D & BAMW2DDUP-W-22698 & $7 / 10 / 08$ & $133.26-152.93$ & ND & ND & ND & Replicate \\
\hline MW9 & BAMW9-W-22678 & $7 / 11 / 08$ & $100-110$ & 1.3 & $0.3 \mathrm{~J}^{\mathrm{b}}$ & ND & Primary \\
\hline MW9 & BAMW9-W-22678DUP & $7 / 11 / 08$ & $100-110$ & 1.3 & $0.3 \mathrm{~J}$ & ND & Duplicate \\
\hline MW11D & BAMW11D-W-22683 & $7 / 10 / 08$ & $125-135$ & $0.9 \mathrm{~J}$ & ND & ND & Primary \\
\hline MW11D & BAMW11D-W-22683DUP & $7 / 10 / 08$ & $125-135$ & $0.8 \mathrm{~J}$ & ND & ND & Duplicate \\
\hline PWS2 & BAPWS2-W-22696 & $7 / 11 / 08$ & 155 & $0.8 \mathrm{~J}$ & ND & ND & Primary \\
\hline PWS2 & BAPWS2-W-22696DUP & $7 / 11 / 08$ & 155 & $0.7 \mathrm{~J}$ & ND & ND & Duplicate \\
\hline QC & BAQCIR-W-22700 & $7 / 11 / 08$ & - & ND & ND & ND & Rinsate after MW10S \\
\hline QC & BAQCIR-W-22701 & $7 / 11 / 08$ & - & ND & ND & ND & Rinsate after MW8 \\
\hline QC & BAQCTB-W-22701 & $7 / 10 / 08$ & - & ND & ND & ND & Trip blank \\
\hline QC & BAQCTB-W-22702 & $7 / 12 / 08$ & - & ND & ND & ND & Trip blank \\
\hline
\end{tabular}

a ND, contaminant not detected at an instrument detection limit of $0.1 \mu \mathrm{g} / \mathrm{L}$.

b Qualifier J indicates an estimated concentration below the method quantitation limit of $1.0 \mu \mathrm{g} / \mathrm{L}$. 


\section{Appendix C:}

Sample Documentation from TestAmerica Laboratories, Inc. 


\section{TestAmerica \\ THE LEADER IN ENYIRONMENTAL TESTING}

July 29,2008

TestAmerica Laboratories, Inc.

Mr. Clyde Dennis

Argonne National Laboratory

9700 S. Cass Avenue

Building 203, Office B149

Argonne, IL 60439

Re: Laboratory Project No. 21005

Case: BARNES; SDG: 126520

Dear Mr. Dennis:

Enclosed are analytical results for samples that were received by TestAmerica Burlington on July $14^{\text {th }}, 2008$. Laboratory identification numbers were assigned, and designated as follows:

\begin{tabular}{|c|c|}
\hline Lab ID & $\begin{array}{l}\text { Client } \\
\text { Sample ID }\end{array}$ \\
\hline
\end{tabular}

Received: $07 / 14 / 08$ ETR No: 126520

$\begin{array}{llll}759578 & \text { BAMW14S-W-22689 } & 07 / 10 / 08 & \text { WATER } \\ 759579 & \text { BAMW8-W-22676 } & 07 / 11 / 08 & \text { WATER } \\ 759580 & \text { BAMW12D-W-22686 } & 07 / 11 / 08 & \text { WATER } \\ 759581 & \text { BAMW10S-W-22679 } & 07 / 11 / 08 & \text { WATER } \\ 759582 & \text { BAPWS3-W-22697 } & 07 / 11 / 08 & \text { WATER } \\ 759583 & \text { BAQCTB-W-22702 } & 07 / 12 / 08 & \text { WATER } \\ 759854 & \text { VHBLK01 } & 07 / 14 / 08 & \text { WATER }\end{array}$

Documentation of the condition of the samples at the time of their receipt and any exception to the laboratory's Sample Acceptance Policy is documented in the Sample Handling section of this submittal. The samples, as received, were not acid preserved. On that basis the laboratory did provide for the analytical work to be performed within seven days of sample collection.

In order to accommodate field length limitations in processing the data summary forms, the laboratory did, in certain instances, abbreviate the sample identifier. The electronically formatted data provides for the full sample identifier.

\section{SOM01.2 Volatile Organics (Trace Level Water)}

A storage blank was prepared for volatile organics analysis, and stored in association with the storage of the sample. That storage blank, identified as VHBLK01, was carried through the holding period with the samples, and analyzed.

\section{1}

30 Community Drive Suite 11 South Burlington, VT 05403 tel 802.660 .1990 fax 802.660 .1919 www.testamericainc.com 
Sample BAMW10S-W-22679 was analyzed at a 5.2-fold dilution, based on the results of preliminary screening. An additional, more concentrated analysis was performed on the sample in order to provide for a lower reporting of possible target analyte constituents that were not identified in the primary analysis. That analysis was performed without a dilution. Both sets of results for the analysis of sample BAMW10S-W-22679 are included in this submittal. Each of the analyses associated with the sample set exhibited an acceptable internal standard performance. There was an acceptable recovery of each deuterated monitoring compound (DMC) in the analysis of the method blank and the analysis of the storage blank. The analysis of the samples in this sample set did meet the technical acceptance criteria specific to DMC recoveries, although not all DMC recoveries were within the control range in each analysis. The technical acceptance criteria does provide for the recovery of up to three DMCs to fall outside of the control range in the analysis of field samples. With the exception of that performed on sample BAQCTB-W22702 , the recoveries of 2-butanone- $d_{5}$ and 2 -hexanone- $d_{5}$ were elevated in the analysis of each of the field samples. Matrix spike and matrix spike duplicate analyses were not performed on the samples in this sample set. Trace concentrations of acetone and 1,2,3-trichlorobenzene were identified in the analysis of the method blank associated with the analytical work. The derived concentration of each compound in that analysis was below the established reporting limit, and the analysis did meet the technical acceptance criteria for a compliant method blank acquisition. The analysis of the storage blank associated with the sample set was free of target analyte contamination. Present in the storage blank and method blank analyses was a non-target constituent that represented a compound that is related to either the DMC formulation or to column bleed. The fact that the presence of this compound is not within the laboratory's control is at issue. The derived results for that compound have been qualified with an " $X$ " qualifier to reflect the source of the contamination. An instrument blank was analyzed following the more concentrated analysis of sample BAMW10S-W-22679. A trace concentration of acetone was identified in that analysis at a concentration below the established reporting limit, and the analysis did meet the technical acceptance criteria for a compliant instrument blank acquisition.

The responses for each target analyte met the relative standard deviation criterion in the initial calibration. The response for each target analyte met the percent difference criterion in the continuing calibration check acquisition. The response for each target analyte met the 50.0 percent difference criterion in the closing calibration check acquisition. In the initial calibration and the calibration check acquisitions, the response for acetone did not meet the minimum relative response criterion of 0.010 in each instance. The relative response for acetone was above 0.009 in all instances.

The primary quantitation mass for methylcyclohexane that is specified in the Statement of Work is mass 83 . The laboratory did identify a contribution to mass 83 from 1,2-dichloropropane-d $d_{6}$, one of the deuterated monitoring compounds (DMCs). The laboratory did change the primary quantitation mass assignment to mass 55 for the quantification of methylcyclohexane.

Manual integration was employed in deriving certain of the analytical results. The values that have been derived from manual integration are qualified on the quantitation reports. Extracted ion current profiles for each manual integration are included in the data package, and further documented in the Sample Preparation section of this submittal.

Any reference within this report to Severn Trent Laboratories, Inc. or STL, should be understood to refer to TestAmerica Laboratories, Inc. (formerly known as Severn Trent Laboratories, Inc.) 


\section{TestAmerica}

July 29,2008

THE LEADER IN ENVIRONMENTAL TESTING

Mr. Clyde Dennis

Page 3 of 3

The analytical results associated with the samples presented in this test report were generated under a quality system that adheres to requirements specified in the NELAC standard. Release of the data in this test report and any associated electronic deliverables is authorized by the Laboratory Director's designee as verified by the following signature.

If there are any questions regarding this submittal, please contact me at 802 660-1990.

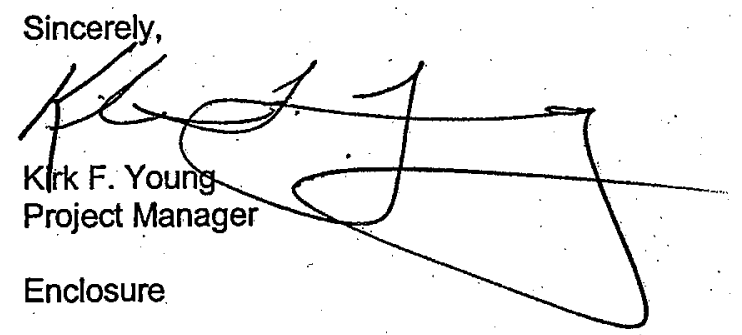




\section{TestAmerica Burlington Data Qualifier Definitions}

\section{Organic}

U: Compound analyzed but not detected at a concentration above the reporting limit.

$\mathrm{J}: \quad$ Estimated value.

$\mathrm{N}$ : Indicates presumptive evidence of a compound. This flag is used only for tentatively identified compounds (TICs) where the identification of a compound is based on a mass spectral library search.

P: $\quad$ SW-846: The relative percent difference for detected concentrations between two GC columns is greater than $40 \%$. Unless otherwise specified the higher of the two values is reported on the Form $\mathrm{I}$.

CLP SOW: Greater than $25 \%$ difference for detected concentrations between two GC columns. Unless otherwise specified the lower of the two values is reported on the Form I.

C: Pesticide result whose identification has been confirmed by GC/MS.

B: Analyte is found in the sample and the associated method blank. The flag is used for tentatively identified compounds as well as positively identified compounds.

E: Compounds whose concentrations exceed the upper limit of the calibration range of the instrument for that specific analysis.

D: Concentrations identified from analysis of the sample at a secondary dilution.

A: Tentatively identified compound is a suspected aldol conden sation product.

$X, Y, Z$ : Laboratory defined flags that may be used alone or combined, as needed. If used, the description of the flag is defined in the project narrative.

\section{Inorganic/Metals}

E: $\quad$ Reported value is estimated due to the presence of interference.

N: Matrix spike sample recovery is not within control limits.

* Duplicate sample analysis is not within control limits.

B: The result reported is less than the reporting limit but greater than the instrument detection limit.

U: Analyte was analyzed for but not detected above the reporting limit.

Method Codes:

P ICP-AES

MS ICP-MS

CV Cold Vapor AA

AS Semi-Automated Spectrophotometric

FQA009:02.18.08:4

TestAmerica Burlington 


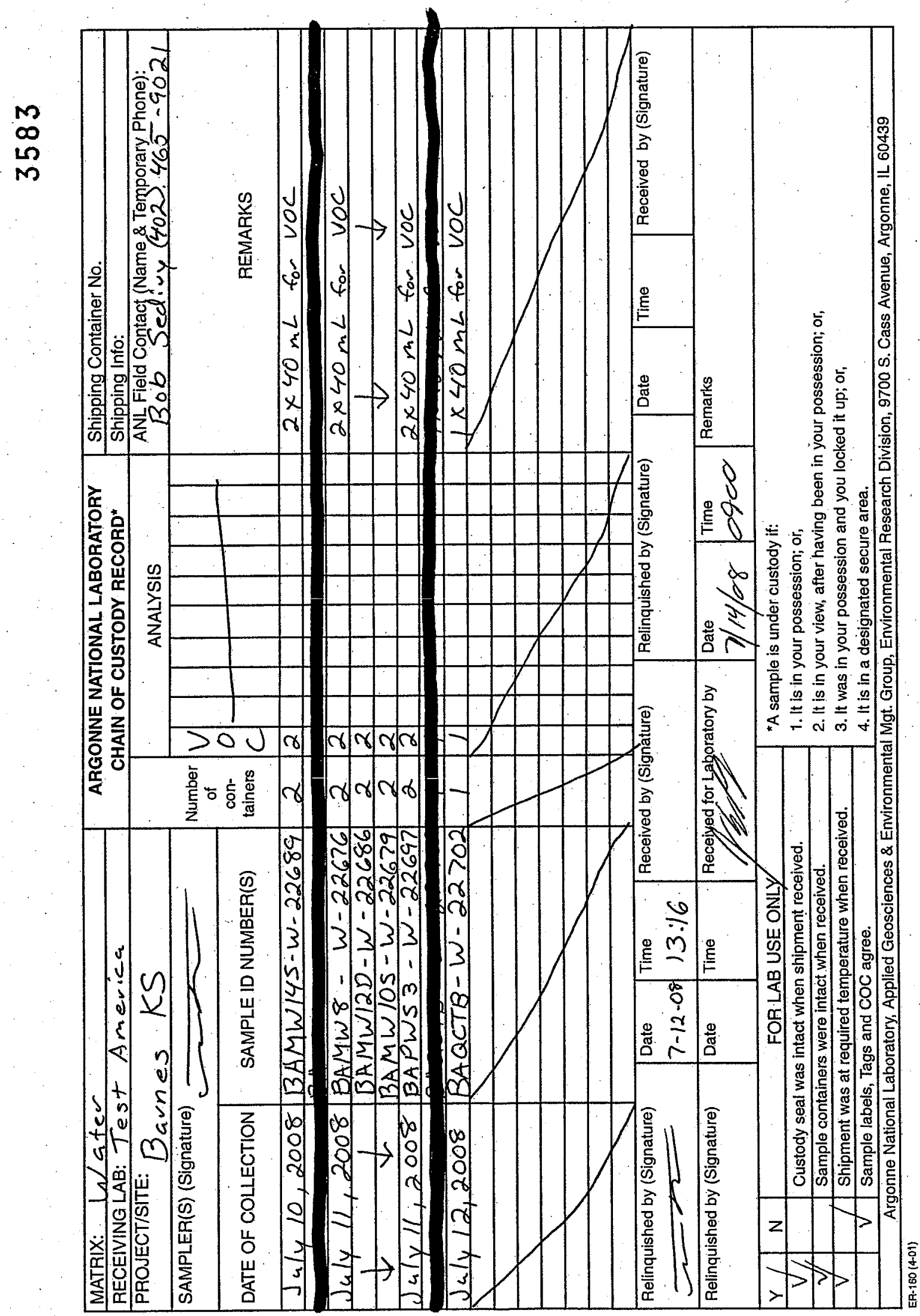


IA - FORM I VOA-I

VOLATILE ORGANICS ANALYSIS DATA SHEET

Lab Name: TestAmerica BURLINGTON

Contract: 21005

Lab Code: STLV Case No.: BARNES

Matrix: (SOIL/SED/WATER) Water

Mod. Ref No.:

SDG No.: 126520
Sample wt/vol: $25.0 \quad(\mathrm{~g} / \mathrm{mL}) \mathrm{mL}$

Level : (TRACE/LOW/MED) TRACE

$\%$ Moisture: not dec.

GC Column: DB-624

Soil Extract Volume:

Purge Volume: 25.0

ID : 0.53
Lab Sample ID: 759581

Lab File ID: 759581 I2

Date Received: 07/14/2008

Date Analyzed: 07/15/2008

(mm)

Dilution Factor: 1.0

(uL) Soil Aliquot Volume:

(uL)

(mE)

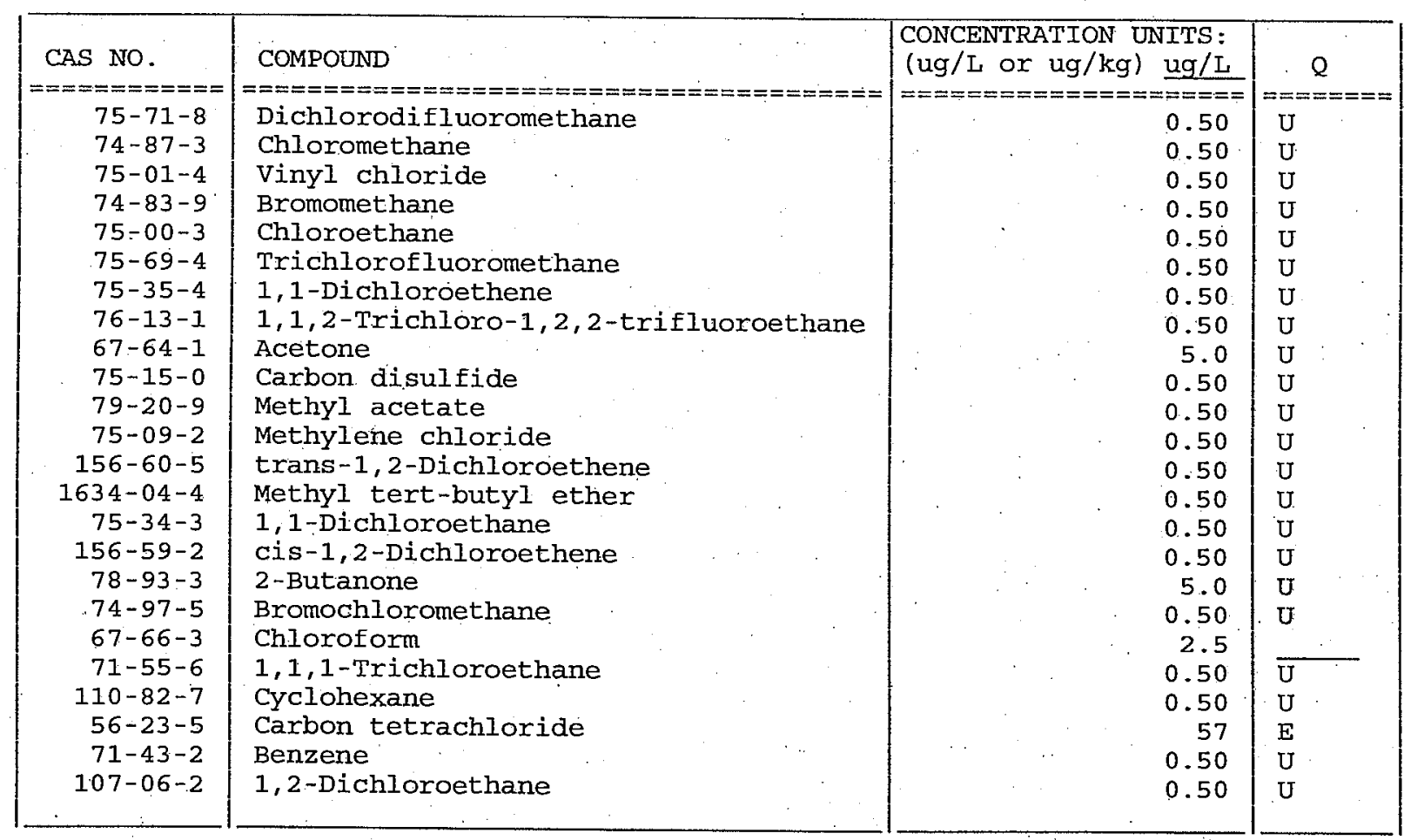

Report 1,4-Dioxane for Low-Medium VOA analysis only 
Lab Name: TestAmerica BURLINGTON

Lab Code: STLV Case No.: BARNES

Matrix: (SOIL/SED/WATER) Water

Sample wt/vol: 25.0

$(\mathrm{g} / \mathrm{mL}) \mathrm{mL}$

. Level：(TRACE/LOW/MED) TRACE

$\%$ Moisture: not dec.

GC Column: DB-624

ID $: 0.53$

Soil Extract Volume:

Purge Volume: 25.0
Contract: 21005

Mod. Ref No.:
SDG NO.: 126520
Lab Sample ID: 759581

Lab File ID: $759581 \mathrm{I} 2$

Date Received: 0.7./14/2008

Date Analyzed: 07/15/2008

Dilution Factor: 1.0

Soil Aliquot Volume:

(uL)

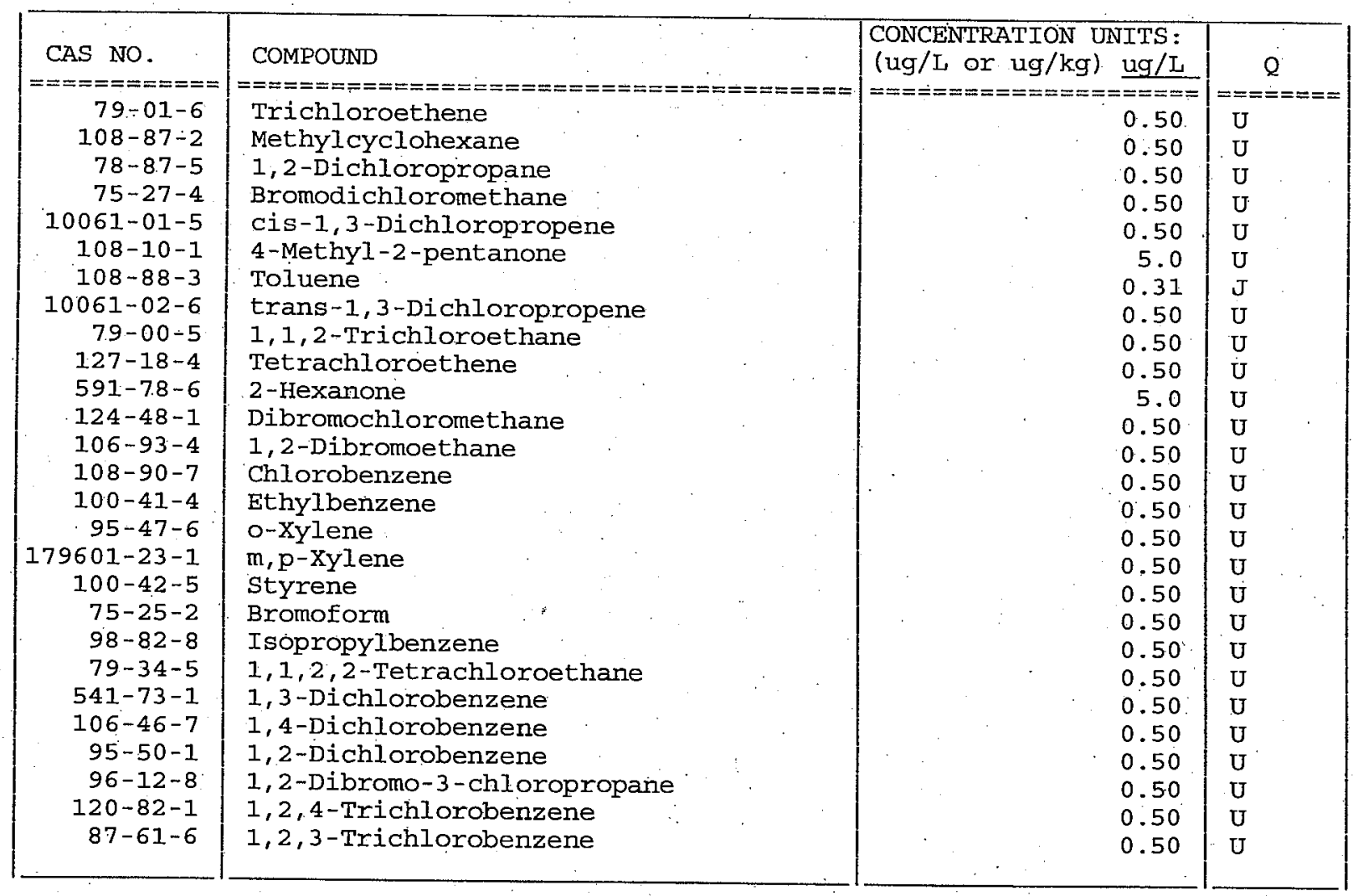


$1 A$ - FORM I VOA-1

VOLATILE ORGANICS ANALYSIS DATA SHEET

EPA SAMPLE NO. MW10S22679DL

Lab Name: TestAmerica BURLINGTON

Contract: 21005

Lab Code: STLV. Case No.: BARNES

Mod. Ref No.:

SDG No.: 126520

Matrix: (SOIL/SED/WATER) Water

Lab Sample ID: 759581D1

Sample wt/vol: 25.0

$(\mathrm{g} / \mathrm{mL}) \mathrm{mL}$

Lab File ID: 759581D

Level: (TRACE/LOW/MED) TRACE

Date Received: 07/14/2008

$\%$ Moisture: not dec.

Date Analyzed: 07/1.5/2008

GC Column: DB-624

ID $: 0.53 \quad(\mathrm{~mm})$

Dilution Factor: 5.2

Soil Extract Volume:

(uL)

Soil Aliquot Volume:

(uL)

Purge Volume: 25.0 .

(mL)

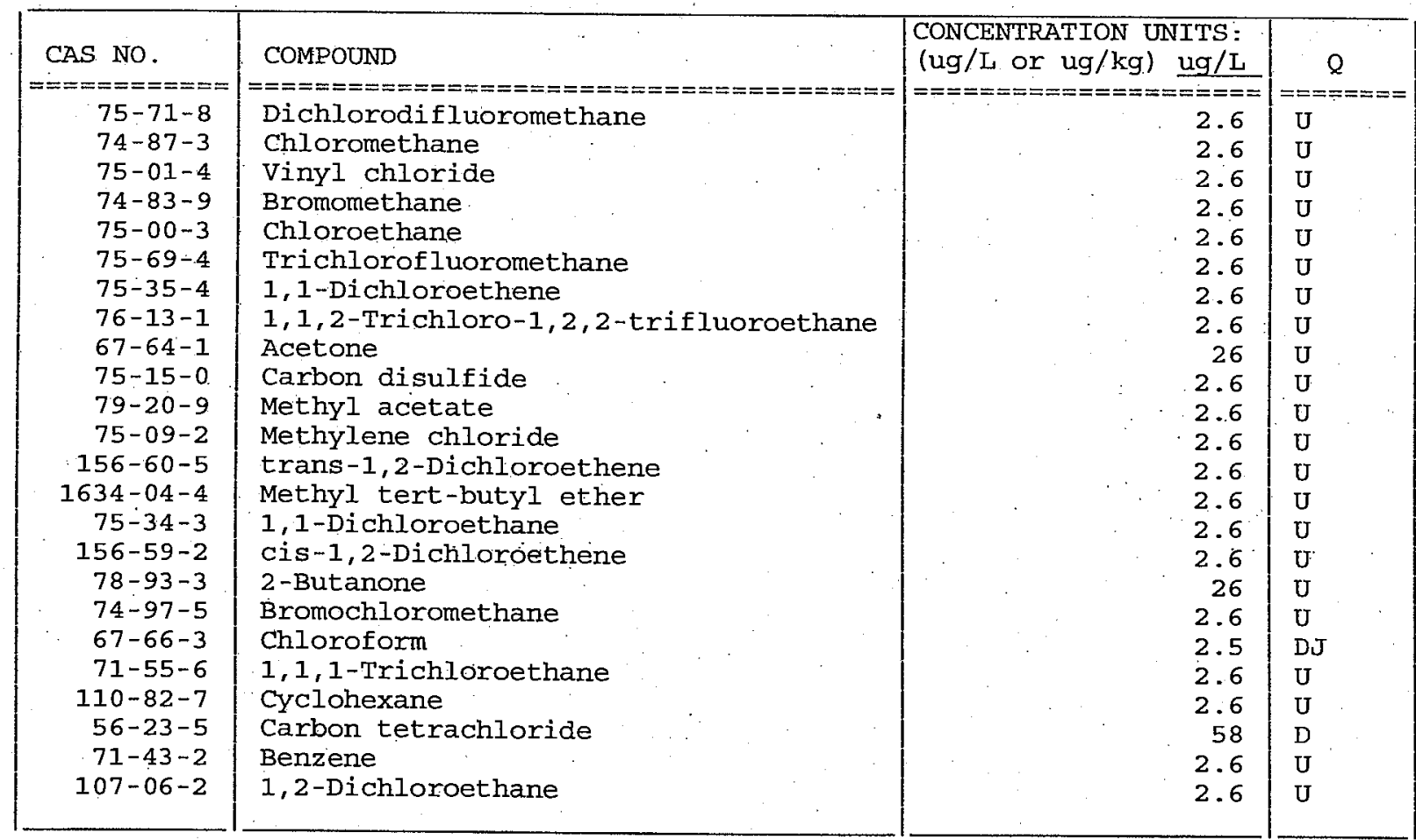

Report 1,4-Dioxane for Low-Medium VOA analysis only 
$1 B$ - FORM I VOA-2

VOLATILE ORGANICS ANALYSIS DATA SHEET

Lab Name: TestAmerica BURLINGTON

Lab Code: STLV Case No.: BARNES

Matrix: (SOIL/SED/WATER) Water

Sample wt/vol: 25.0

$(g / m L) m L$

Level: (TRACE/LOW/MED). TRACE

\% Moisture: not dec.

GC Column: DB-624

ID $: 0.53$

Soil Extract Volume:

Purge Volume: 25.0
Contract : 21005

Mod. Ref No. :
(mL)
EPA SAMPLE NO.

MWW10S22679DI
Lab Sample ID: 759581D1

Lab File ID: 759581D

Date Received: 07/14/2008

Date Analyzed: 07/15/2008

Dilution Factor: 5.2

Soil Aliquot Volume:

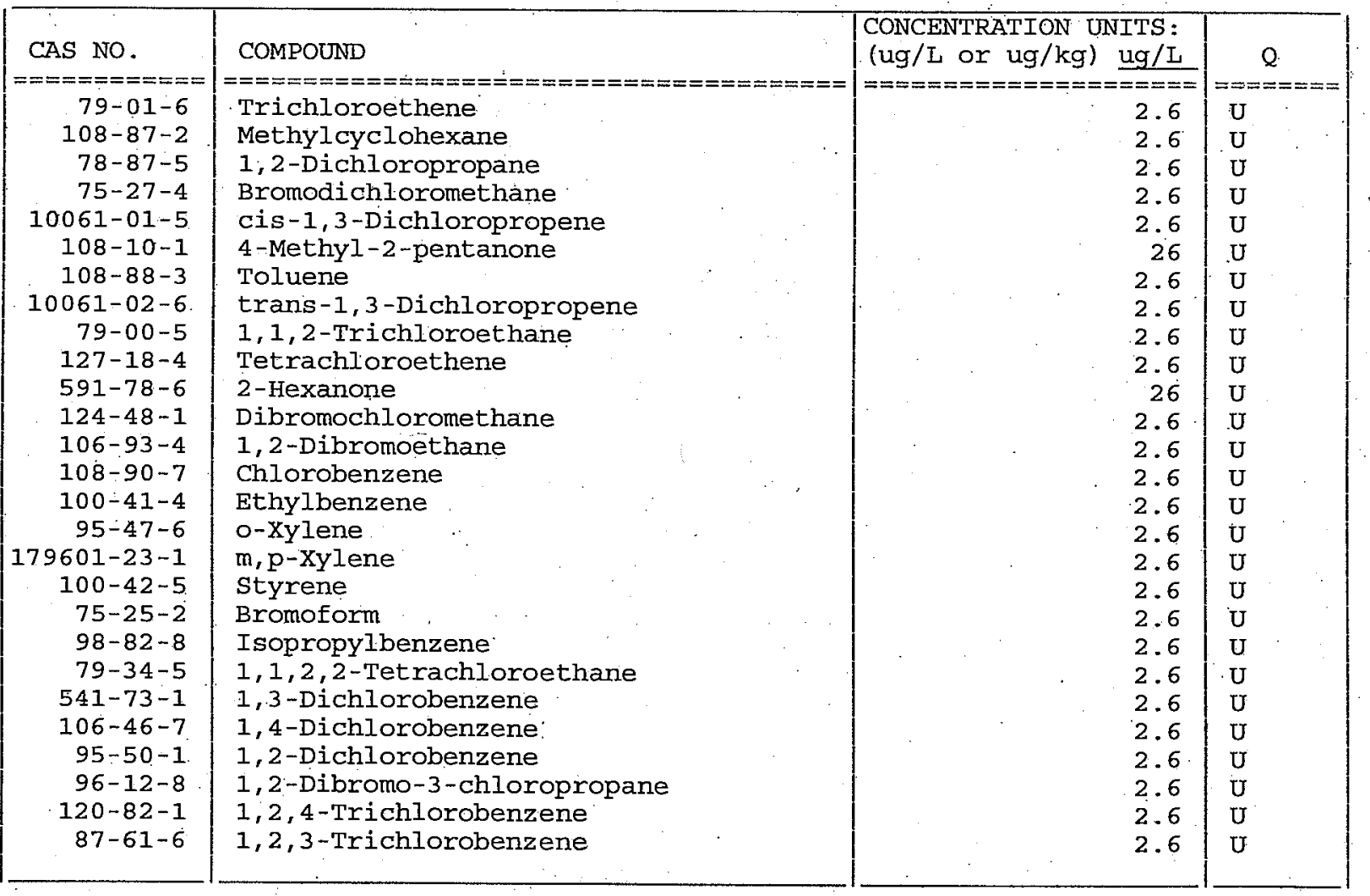


IA - FORM I VOA-1

VOLATILE ORGANICS ANALYSIS DATA SHEET
EPA SAMPLE NO.

MW12D22686
Lab Name: TestAmerica BURIINGTON

Lab Code: STLV
Matrix: (SOIL/SED/WATER) Water

Sample wt/vol: 25.0

$(\mathrm{g} / \mathrm{mL}) \mathrm{mL}$

Level: (TRACE/LOW/MED) TRACE

\% Moisture: not dec.

GC Column: $\mathrm{DB}-624$

ID : 0.53

Soil Extract Volume:

Purge Volume: 25.0
Contract : 21005

SDG No. : 126520

Mod. Ref No. :

Lab Sample ID: 759580

Lab File ID: 759580

Date Received: 07/14/2008

Date Analyzed: 07/15/2008

Dilution Factor: 1.0

Soil Aliquot Volume:

(UL)

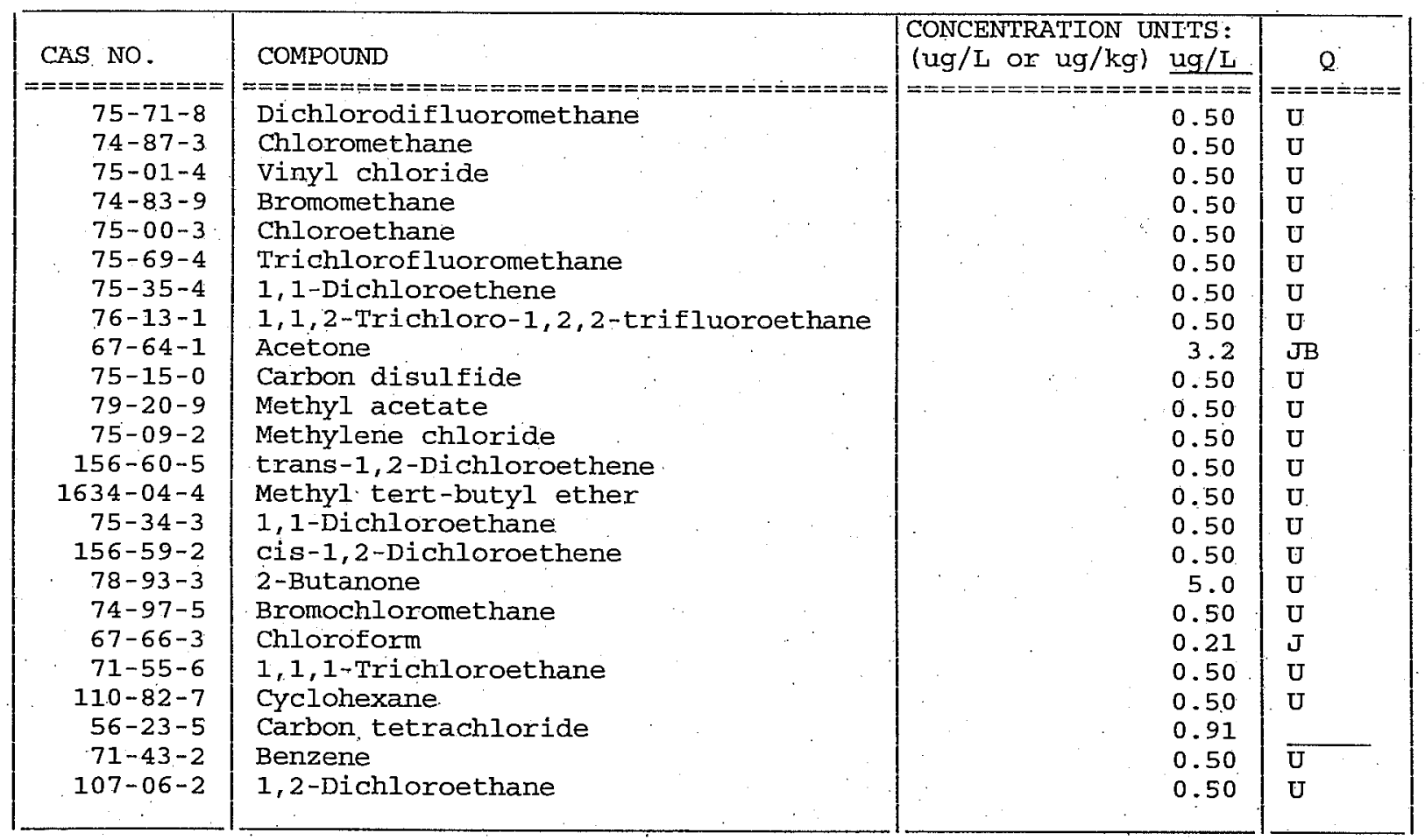

Report 1,4-Dioxane for Low-Medium VOA analysis only 
IB - FORM I VOA-2

VOLATILE ORGANICS ANALYSIS DATA SHEET

EPA SAMPLE NO.

Lab Name: TestAmerica BURLINGTON

Contract: 21005

Lab Code: STLV Case No.: BARNES

Mod. Ref No.:

SDG No.: 126520

Matrix: (SOIL/SED/WATER) Water

Sample wt/vol: 25.0

$(g / m L) m L$

Level: (TRACE/LOW/MED) TRACE

\% Moisture: not dec.

GC Column: DB-624

ID : 0.53

(mm)

(uL)

Lab Sample ID: 759580

Lab File ID: 759580

Date Received: 07/14/2008

Date Analyzed: 07/15/2008

Soil Extract Volume:

Dilution Factor: 1.0

Purge Volume: 25.0

$(\mathrm{mL})$

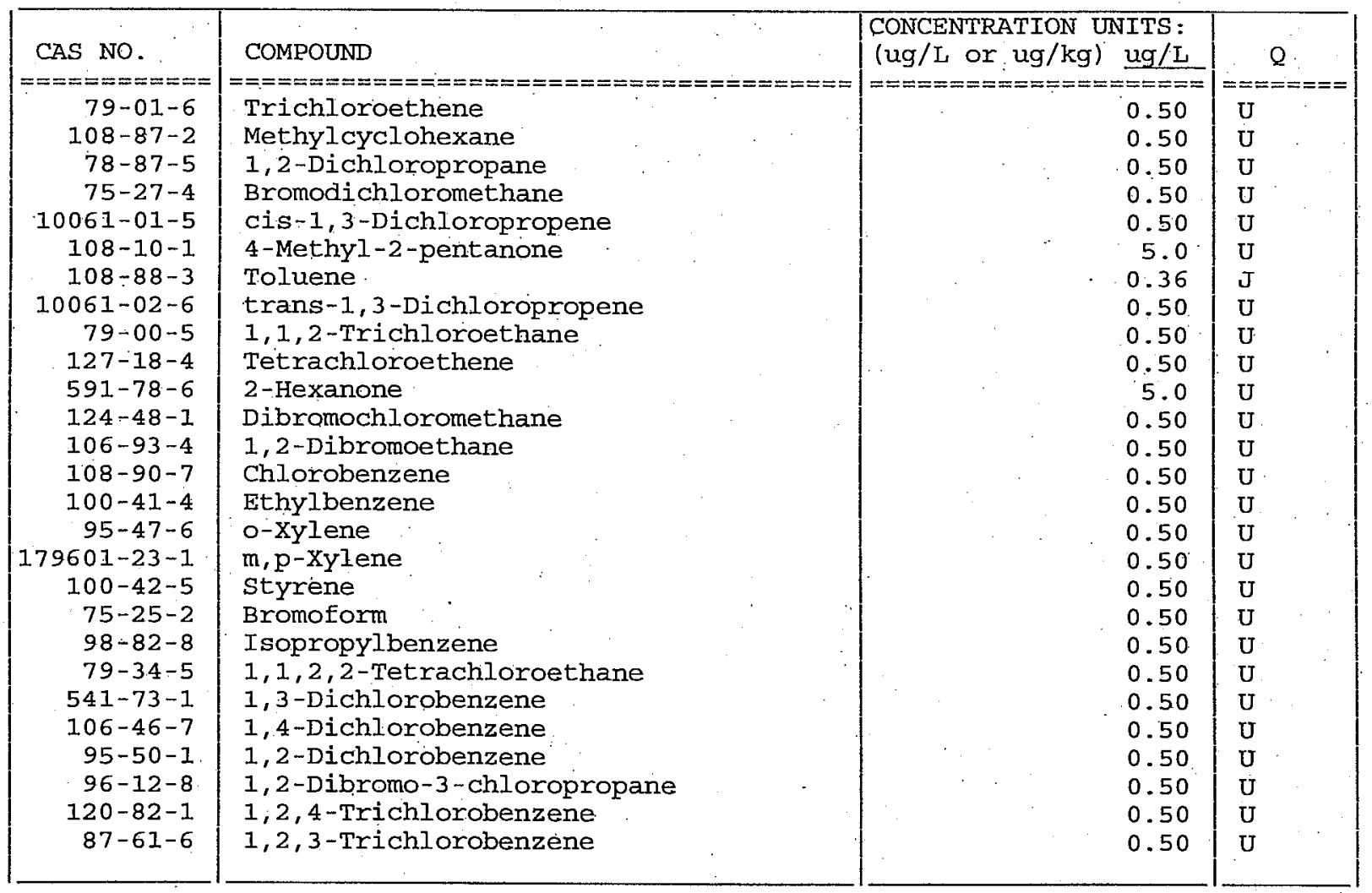


$1 A$ - FORM I VOA-1

VOLATILE ORGANICS ANALYSIS DATA SHEET

EPA SAMPLE NO.

Lab Name: TestAmerica BURLINGTON

Contract $: 21005$

Lab Code: STLV Case No.: BARNES

Mod. Ref No.:

SDG No.: 126520

Matrix: (SOIL/SED/WATER) Water

Sample wt/vol: 25.0

$(\mathrm{g} / \mathrm{mL}) \mathrm{mL}$

Level: (TRACE/LOW/MED) TRACE

$\div$ Moisture: not dec.

GC Column: DB-624

ID: 0.53

Soil Extract Volume:

Purge Volume: 25.0
Lab Sample ID: 759578

Lab File.ID: 759578

Date Received: 07/14/2008

Date Analyzed: 07/15/2008

(mm) Dilution Factor: 1.0

(uL): Soil Aliquot Volume:

(uL)

(mL)

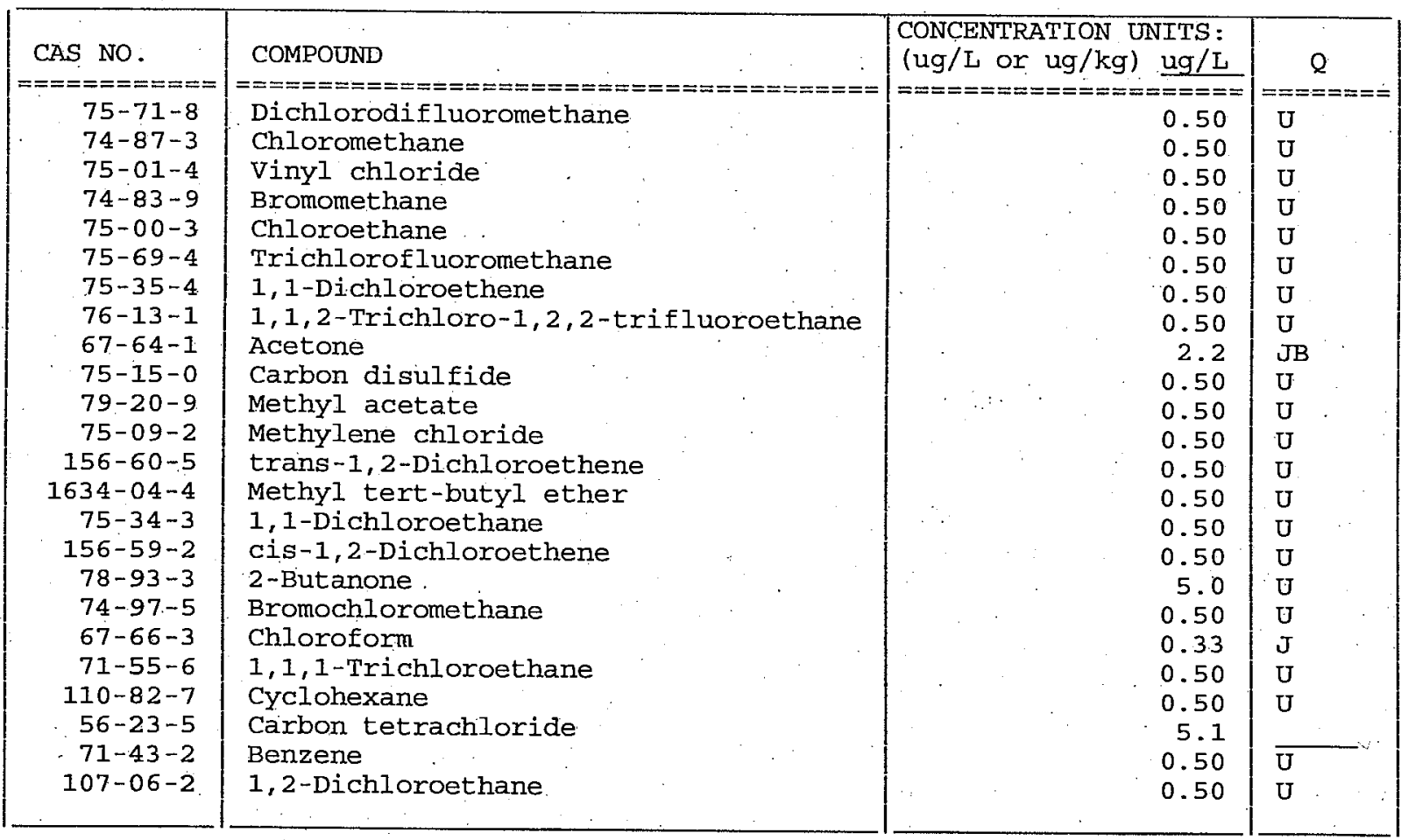

Report 1,4-Dioxane for Low-Medium VOA analysis only 
IB - FORM I VOA-2

VOLATILE ORGANICS ANALYSIS DATA SHEET

EPA SAMPLE NO.

Lab Name: TestAmerica BURLINGTON

Contract: 21005

Lab Code: STLV. Case No.: BARNES

Mod.. Ref No.:

SDG No.: 126520

Matrix: (SOIL/SED/WATER) Water

Sample wt/vol: 25.0

$(\mathrm{g} / \mathrm{mL}) \mathrm{mL}$

Level: (TRACE/LOW/MED) TRACE

\% Moisture: not dec.

GC Column: DB-624

ID $: 0.53$

(mm)

(uL)

(mL)

Purge Volume: 25.0
Lab Sample ID: 759578

Lab File ID: 759578

Date Received: 07/14/2008

Date Analyzed: 07/15/2008

Dilution Factor: 1.0

Soil Aliquot Volume:

(uL)

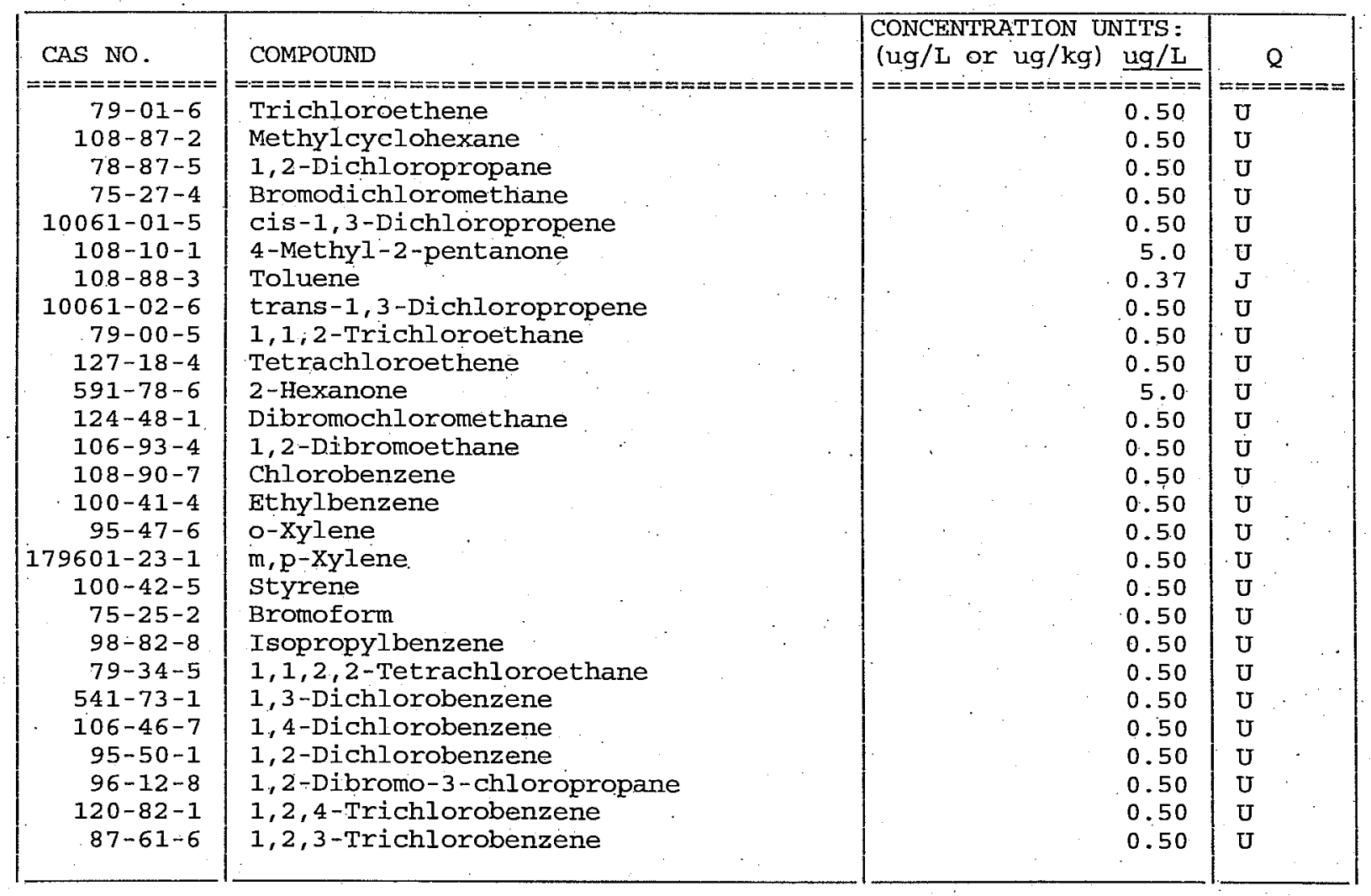


IA - FORM I VOA-1

VOLATILE ORGANICS ANALYSIS DATA SHEET
EPA SAMPLE NO.

MW82267.6
Lab Name: TestAmerica BURLINGTON

Lab Code: STLV

Case No.: BARNES

Matrix: (SOIL/SED/WATER) Water

Sample wt/vol: 25.0

$(\mathrm{g} / \mathrm{mL}) \mathrm{mL}$

Level: (TRACE/LOW/MED) TRACE

\% Moisture: not dec.

GC Column: DB-624

ID : 0.53

Contract: 21005

Mod. Ref No. :

SDG No.: 126520

Lab Sample ID: 759579

Lab File ID: 759579 I2

Date Received: 07/14/2008

Date Analyzed: 07/15/2008

Soil Extract Volume:

(min)

Dilution Factor: 1.0

(u山)

Soil Aliquot Volume:

(uL)

Purge volume: 25.0

(mL)

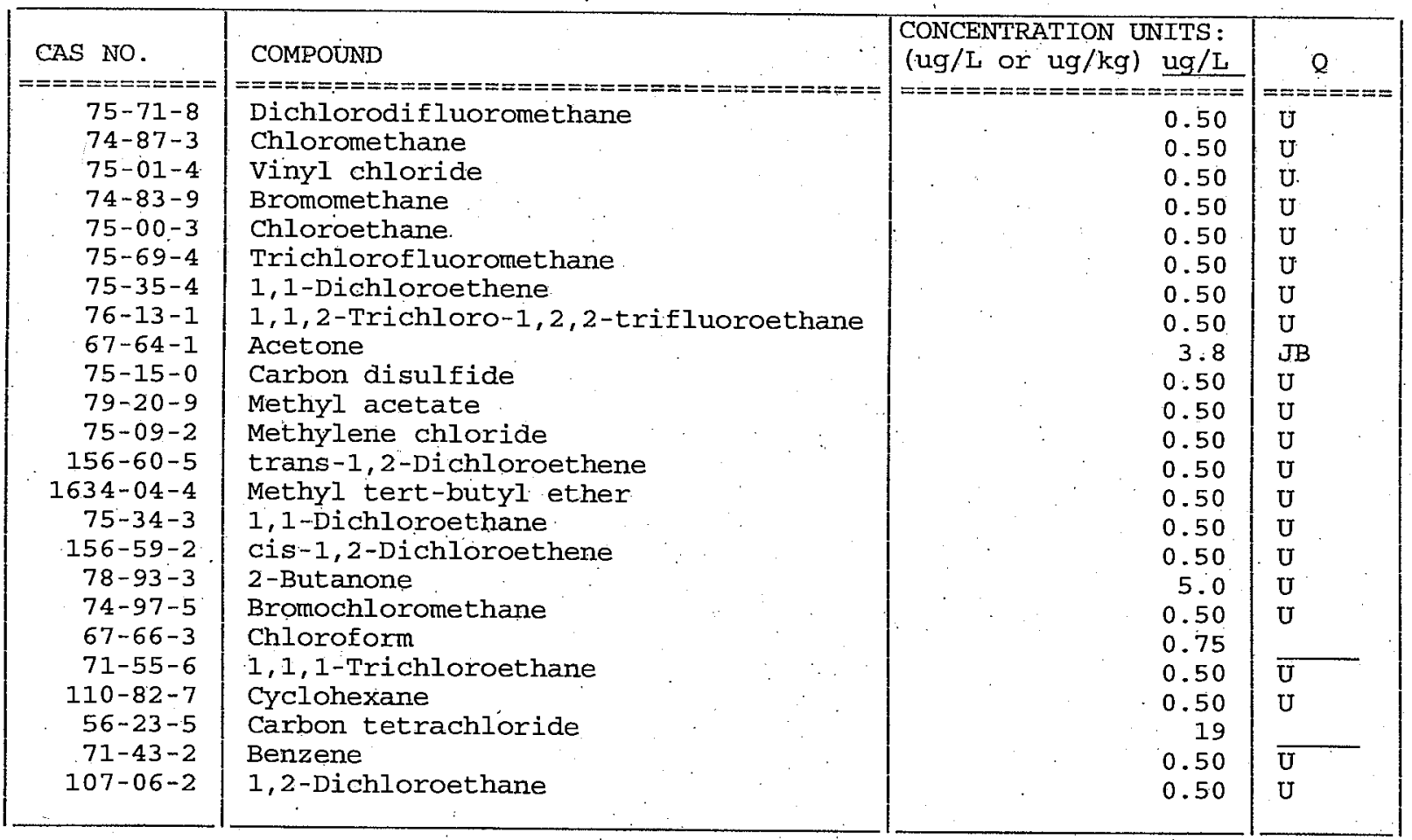

Report 1,4-Dioxane for Low-Medium VOA analysis only 
$1 B$ - FORM I VOA-2

VOLATILE ORGANICS ANALYSIS DATA SHEET

EPA SAMPLE NO.

MW822676

Lab Name: TestAmerica BURLINGTON

Contract: 21005

Lab Code: STLV Case No.: BARNES

Mod. Ref No.:

SDG NO.: 126520

Matrix: (SOIL/SED/WATER) Water

Sample wt/vol: $25.0 \quad(\mathrm{~g} / \mathrm{mL}) \mathrm{mL}$

Level : (TRACE/LOW/MED) TRACE

$\div$ Moisture: not dec.

GC Column: DB-624

Soil Extract Volume:

ID $: 0.53$

(mm)

(uL)

(uL)

Purge Volume: 25.0

(mL)

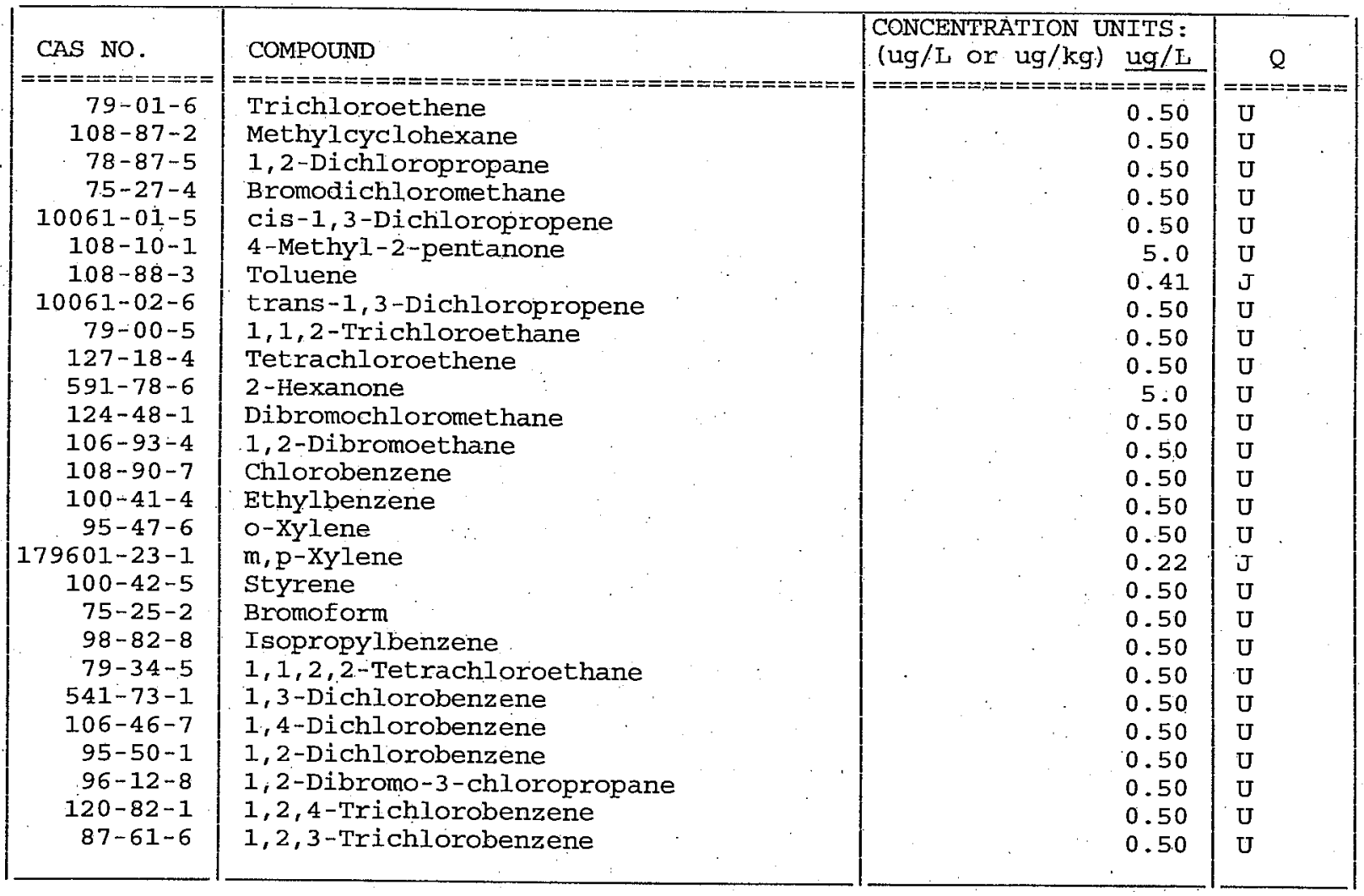


$1 A$ - FORM I VOA-1

VOLATILE ORGANICS ANALYSIS DATA SHEET

Contract: 21005

Mod. Ref No.:
EPA SAMPLE NO.

PWS322697

Lab Name: TestAmericia BURLINGTON

Case No.: BARNES
Matrix: (SOIL/SED/WATER) Water

Sample wt/vol: 25.0

$(\mathrm{g} / \mathrm{mL}) \mathrm{mL}$

Level: (TRACE/LOW/MED) TRACE

․․ Moisture: not dec.

GC Column: DB-624

ID : 0.53

Soil Extract Volume:

Purge Volume: 25.0
Lab Sample ID: 759582

Lab File ID: 759582

Date Received: 07/14/2008

Date Analyzed: 07/15/2008

Dilution Factor: 1.0

(uJ) Soil Aliquot Volume:

(uJ)

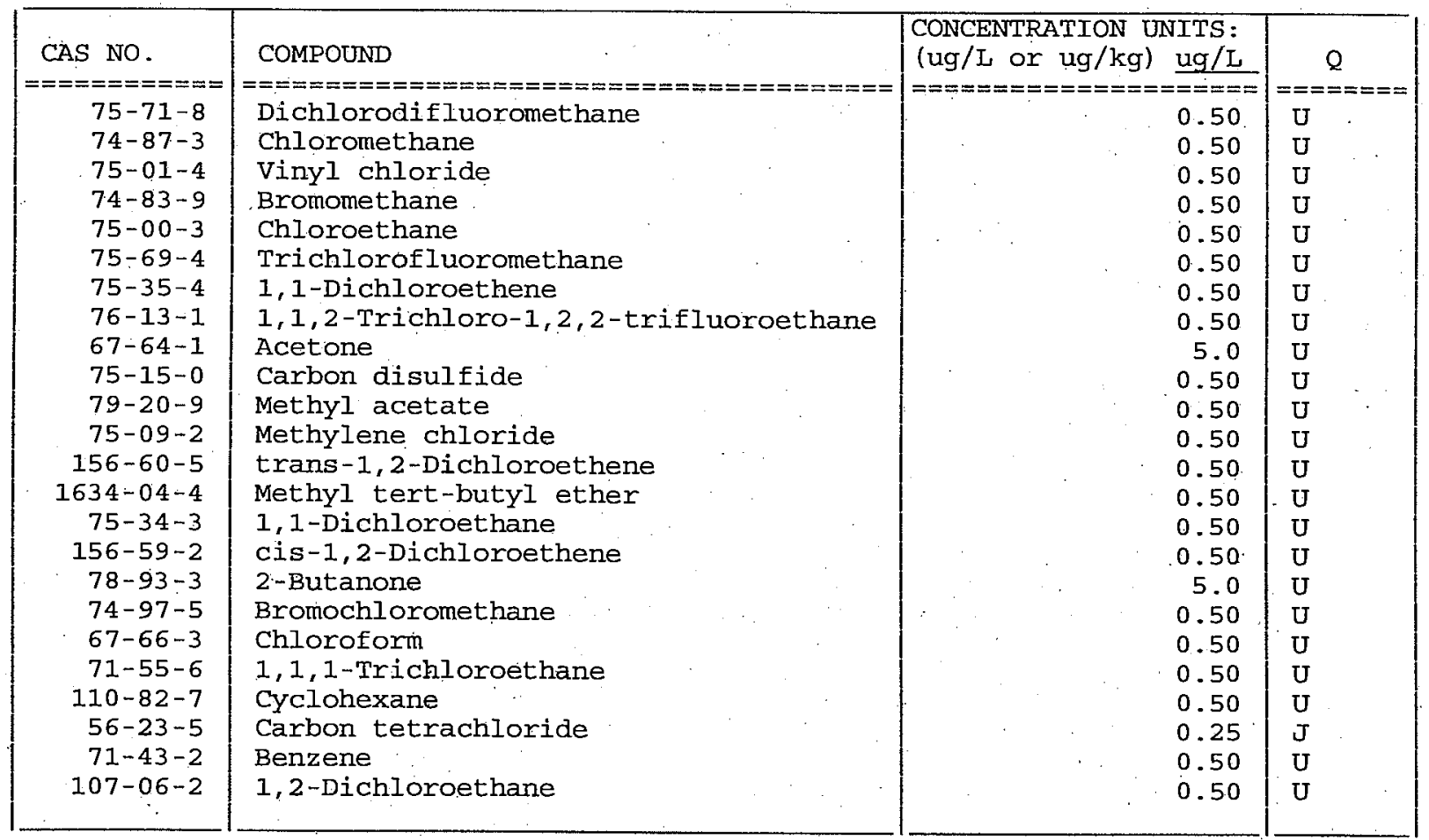

Report 1,4-Dioxane for Low-Medium VOA analysis only 
$1 B$ - FORM I VOA-2

VOLATILE ORGANICS ANALYSIS DATA SHEET

EPA SAMPLE NO.

Lab Name: TestAmerica BURLINGTON

Contract : 21005

Lab Code: STLV Case No.: BARNES

Mod. Ref No.:

SDG NO.: 126520

Matrix: (SOIL/SED/WATER) Water

Lab Sample ID: 759582

Sample wt/vol: $25.0(\mathrm{~g} / \mathrm{mL}) \mathrm{mL}$

Lab File ID: 759582

Level: (TRACE/LOW/MED) TRACE

Date Received: 07/14/2008

\% Moisture: not dec.

Date Analyzed: 07/15/2008

GC Column: DB-624

ID $: 0.53$

$(\mathrm{mm})$

Dilution Factor: 1.0

Soil Extract Volume:

(uL) Soil Aliquot Volume:

(uL)

Purge Volume: 25.0

(mL)

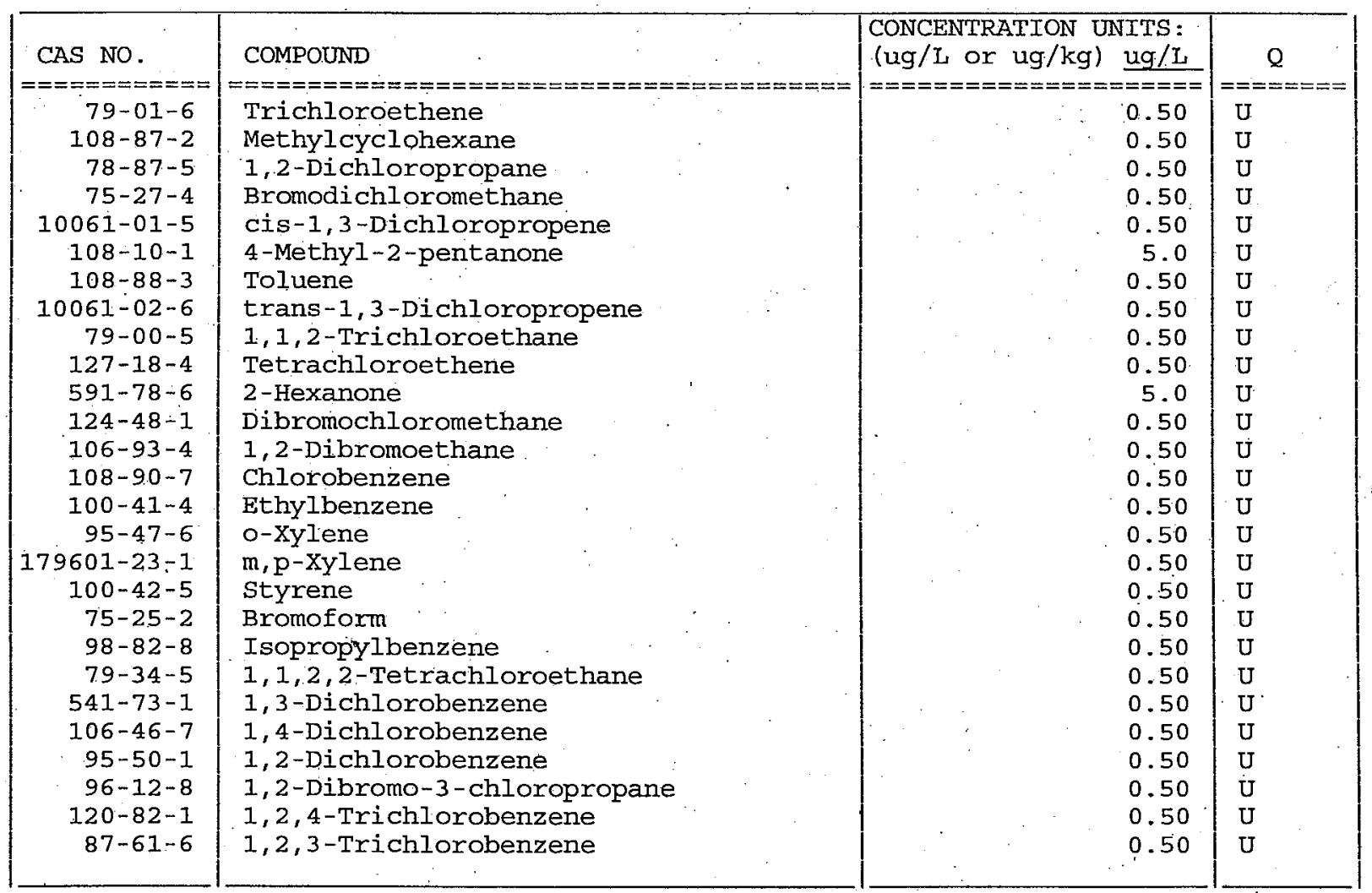


$1 A$ - FORM I VOA-1

VOLATILE ORGANICS ANALYSIS DATA SHEET

EPA SAMPLE NO.

QCTB22702

Lab Name: TestAmerica BURLINGTON

Contract: 21005

Lab Code: STLV Case No.: BARNES

Mod. Ref No. :

SDG No.: 126520

Matrix: (SOIL/SED/WATER) Water

Sample wt/vol: 25.0

$(\mathrm{g} / \mathrm{mL}) \mathrm{mI}$

Level: (TRACE/LOW/MED) TRACE

\% Moisture: not dec.

GC Column: DB-624

ID : 0.53

Soil Extract Volume:

Purge Volume: 25.0
Lab Sample ID: 759583 .

Lab File ID: 759583

Date Received: 07/14/2008

Date Analyzed: 07/15/2008

Dilution Factor: 1.0

Soil Aliquot Volume:

(uL)

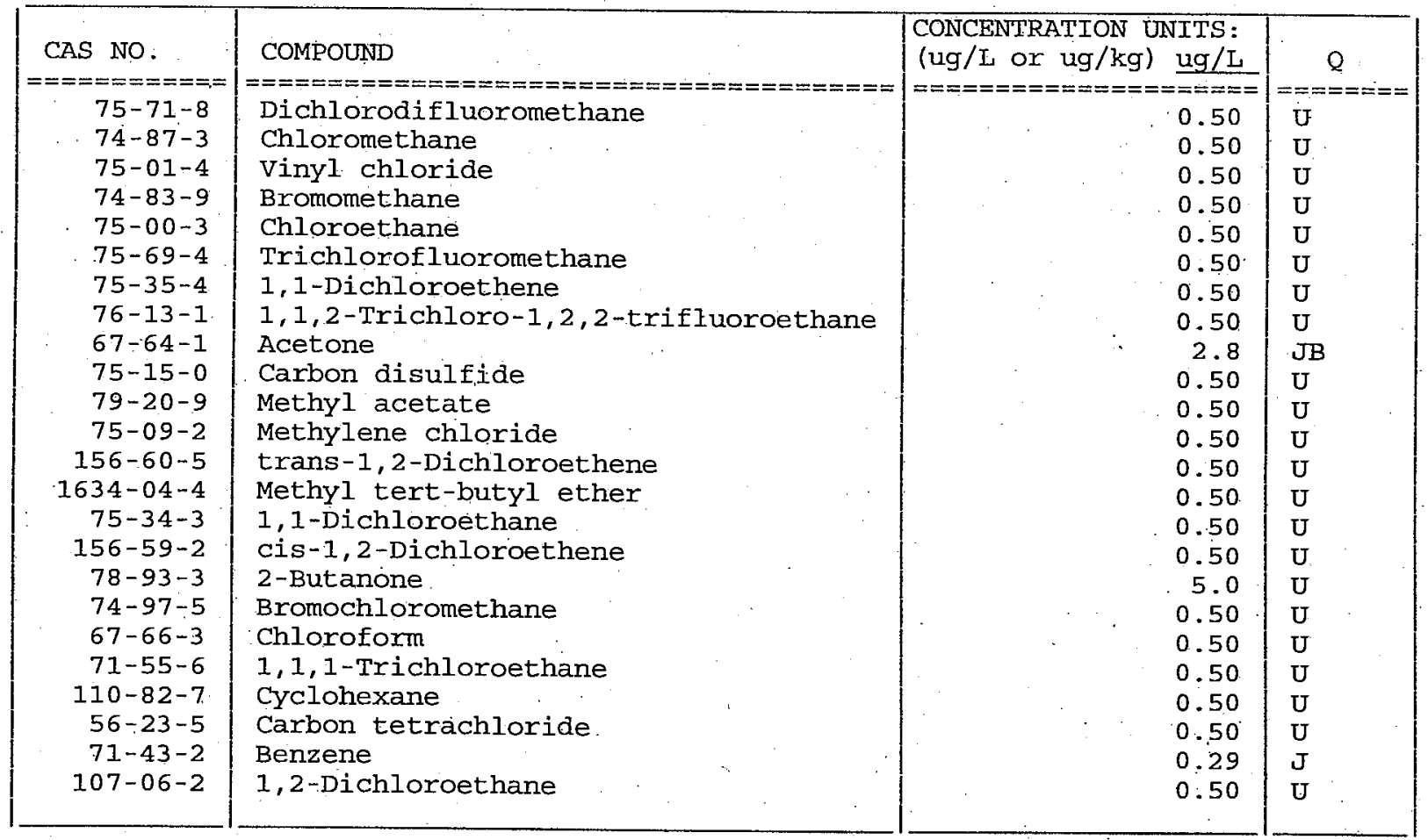

Report 1,4-Dioxane for Low-Medium VOA analysis only 
IB - FORM I VOA-2

VOLATILE ORGANICS ANALYSIS DATA SHEET
EPA SAMPLE NO.

QCTB22702
Lab Name: TestAmerica BURLINGTON

Lab code: STLV

Case No.: BARNES

Matrix: (SOIL/SED/WATER) Water

Sample wt/vol: 25.0

$(\mathrm{g} / \mathrm{mL}) \mathrm{mL}$

Level: (TRACE/LOW/MED) TRACE

\% Moisture: not dec.

GC Column: DB-624

ID $: 0.53$

Soil Extract Volume:

Purge volume: 25.0
Contract: 21005

Mod. Ref No.:
SDG No. : 126520

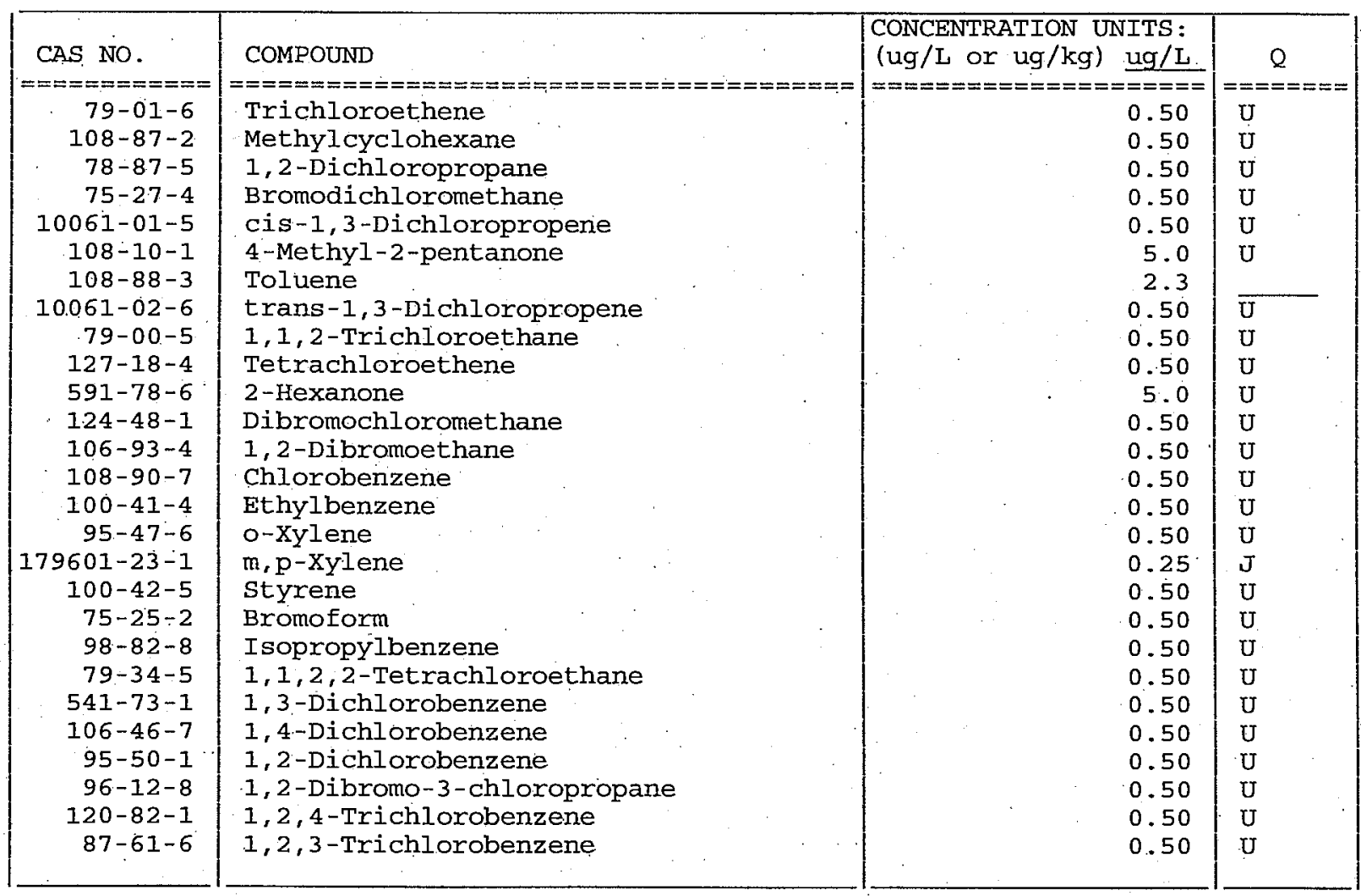


Argonne

Environmental Science Division

Argonne National Laboratory

9700 South Cass Avenue, Bldg. 203

Argonne, IL 60439-4843

www.anl.gov

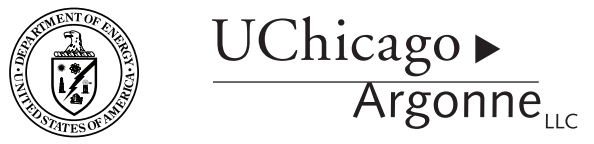

A U.S. Department of Energy laboratory

managed by UChicago Argonne, LLC 